\title{
Condensation of ortho-phenylenediamines and phenylhydrazines with ethyl 4-chloro-3-oxobutanoate: A facile approach for the synthesis of substituted $1 H$-benzimidazoles, pyrazolones and pyrazoles
}

\author{
Dayakar Cherupally, Jyothi Dondra, Suman Pathi, China Raju Bhimapaka* \\ Natural Products Chemistry Division, CSIR-Indian Institute of Chemical Technology, \\ Hyderabad-500 007, India (phone: +914027161725; fax: +91402716512). \\ e-mail: chinaraju@,iict.res.in
}

\section{Experimental protocol}

Ortho-phenylenediamines and ethyl 4-chloro-3-oxobutanoate were obtained from SigmaAldrich, phenylhydrazine hydrochlorides and solvents were commercially available. Column Chromatography (CC): silica gel $\left(\mathrm{SiO}_{2} ; 60-120\right.$ mesh). Mp: Mettler-Temp apparatus; uncorrected. IR Spectra: Perkin-Elmer-1600 FT-IR spectrometer; in $\mathrm{KBr} ; v$ in $\mathrm{cm}^{-1} .{ }^{1} \mathrm{H}-$ and ${ }^{13} \mathrm{C}$-NMR spectra: Bruker-Avance-300 spectrometers; solvent $\mathrm{CDCl}_{3}$; chemical shifts $\delta$ in ppm rel. to $\mathrm{Me}_{4} \mathrm{Si}$ as internal standard, $J$ in $\mathrm{Hz}$. MS-ESI: $7070 H$ spectrometer with a direct inlet system; in $m / z$ (rel. \%). ESI-HR-MS: Agilent 6510 Q-TOF LC/MS instrument.

\section{Experimental Data}

2-(Chloromethyl)-1H-benzo[ $d]$ imidazole (3a)

Pale yellow solid; Yield: $82 \%$; Mp 152-154 ${ }^{\circ} \mathrm{C} ;{ }^{1} \mathrm{H}$ NMR $\left(300 \mathrm{MHz}, \mathrm{CDCl}_{3}\right): \delta=4.85(\mathrm{~s}, 2 \mathrm{H}$, $\left.\mathrm{CH}_{2} \mathrm{Cl}\right), 7.19-7.28(\mathrm{~m}, 2 \mathrm{H}, \mathrm{Ar}-\mathrm{H}), 7.55-7.62(\mathrm{~m}, 2 \mathrm{H}, \mathrm{Ar}-\mathrm{H}) ;{ }^{13} \mathrm{C} \mathrm{NMR}\left(75 \mathrm{MHz}, \mathrm{CDCl}_{3}\right) \delta=$

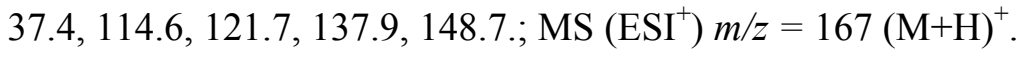

2-(Chloromethyl)-5-nitro-1H-benzo[ $d]$ imidazole (3b)

Pale yellow solid; Yield: $75 \%$; Mp 178-180 ${ }^{\circ} \mathrm{C} ;{ }^{1} \mathrm{H}$ NMR $\left(300 \mathrm{MHz}, \mathrm{CDCl}_{3}\right): \delta=4.87(\mathrm{~s}, 2 \mathrm{H}$, $\left.\mathrm{CH}_{2} \mathrm{Cl}\right), 7.54-7.82(\mathrm{~m}, 1 \mathrm{H}, \mathrm{Ar}-\mathrm{H}), 8.17(\mathrm{~s}, 1 \mathrm{H}, \mathrm{Ar}-\mathrm{H}), 8.38-8.62(\mathrm{~m}, 1 \mathrm{H}, \mathrm{Ar}-\mathrm{H})$; MS (ESI $\left.{ }^{+}\right)$ $m / z=212(\mathrm{M}+\mathrm{H})^{+}$. 
5-Chloro-2-(chloromethyl)-1H-benzo[d]imidazole (3c)

Pale yellow solid; Yield: $72 \%$; Mp 128-130 ${ }^{\circ} \mathrm{C} ;{ }^{1} \mathrm{H}$ NMR $\left(300 \mathrm{MHz}, \mathrm{CDCl}_{3}\right): \delta=4.82(\mathrm{~s}, 2 \mathrm{H}$, $\left.\mathrm{CH}_{2} \mathrm{Cl}\right), 7.18-7.22(\mathrm{~m}, 1 \mathrm{H}, \mathrm{Ar}-\mathrm{H}), 7.41-7.53(\mathrm{~m}, 1 \mathrm{H}, \mathrm{Ar}-\mathrm{H}), 7.57$ (s, 1H, Ar-H); MS (ESI $\left.{ }^{+}\right)$ $m / z=201(\mathrm{M}+\mathrm{H})^{+}$.

(2-(Chloromethyl)-1H-benzo[d]imidazol-5-yl)(phenyl)methanone (3d)

Pale yellow solid; Yield: $60 \%$; Mp 179-181 ${ }^{\circ} \mathrm{C} ;{ }^{1} \mathrm{H}$ NMR $\left(300 \mathrm{MHz}, \mathrm{CDCl}_{3}\right): \delta=4.88(\mathrm{~s}, 2 \mathrm{H}$, $\left.\mathrm{CH}_{2} \mathrm{Cl}\right), 7.44-7.51(\mathrm{~m}, 2 \mathrm{H}, \mathrm{Ar}-\mathrm{H}), 7.56-7.68(\mathrm{~m}, 2 \mathrm{H}, \mathrm{Ar}-\mathrm{H}), 7.78-7.84(\mathrm{~m}, 2 \mathrm{H}, \mathrm{Ar}-\mathrm{H}), 8.08(\mathrm{~s}$, $1 \mathrm{H}, \mathrm{Ar}-\mathrm{H}) ; \mathrm{MS}\left(\mathrm{ESI}^{+}\right) m / z=271(\mathrm{M}+\mathrm{H})^{+}$.

2-(Chloromethyl)-5-methyl-1H-benzo[ $d]$ imidazole (3e)

Pale yellow solid; Yield: $65 \%$; Mp 148-150 ${ }^{\circ} \mathrm{C} ;{ }^{1} \mathrm{H}$ NMR $\left(300 \mathrm{MHz}, \mathrm{CDCl}_{3}\right): \delta=2.57(\mathrm{~s}, 3 \mathrm{H}$, $\left.\mathrm{CH}_{3}\right), 4.85\left(\mathrm{~s}, 2 \mathrm{H}, \mathrm{CH}_{2} \mathrm{Cl}\right), 7.0-7.21(\mathrm{~m}, 2 \mathrm{H}, \mathrm{Ar}-\mathrm{H}), 7.40(\mathrm{~s}, 1 \mathrm{H}, \mathrm{Ar}-\mathrm{H}) ; \mathrm{MS}\left(\mathrm{ESI}^{+}\right) \mathrm{m} / z=181$ $(\mathrm{M}+\mathrm{H})^{+}$.

2-(Chloromethyl)-5,6-dimethyl-1H-benzo[ $d]$ imidazole (3f)

Pale yellow solid; Yield: $62 \%$; Mp 153-155 ${ }^{\circ} \mathrm{C} ;{ }^{1} \mathrm{H}$ NMR $\left(300 \mathrm{MHz}, \mathrm{CDCl}_{3}\right): \delta=2.36(\mathrm{~s}, 6 \mathrm{H}$, $\left.2 \mathrm{CH}_{3}\right), 4.82\left(\mathrm{~s}, 2 \mathrm{H}, \mathrm{CH}_{2} \mathrm{Cl}\right), 7.33(\mathrm{~s}, 1 \mathrm{H}, \mathrm{Ar}-\mathrm{H}) ; \mathrm{MS}_{\left(\mathrm{ESI}^{+}\right)} \mathrm{m} / z=195(\mathrm{M}+\mathrm{H})^{+}$.

3-(Chloromethyl)-1-phenyl-1H-pyrazol-5(4H)-one (5a)

Pale yellow solid; Yield: 62\%; Mp $117-119{ }^{\circ} \mathrm{C}$; IR (KBr) 2795, 1759, 1558, 1407, 1259, 1155, 1032, 761, $696 \mathrm{~cm}^{-1} ;{ }^{1} \mathrm{H}$ NMR (300 MHz, $\left.\mathrm{CDCl}_{3}\right): \delta=3.62\left(\mathrm{~s}, 2 \mathrm{H}, \mathrm{CH}_{2}\right), 4.39(\mathrm{~s}, 2 \mathrm{H}$, $\left.\mathrm{CH}_{2} \mathrm{Cl}\right), 7.21(\mathrm{t}, J=7.4 \mathrm{~Hz}, 1 \mathrm{H}, \mathrm{Ar}-\mathrm{H}), 7.40(\mathrm{t}, J=7.3 \mathrm{~Hz}, 2 \mathrm{H}, \mathrm{Ar}-\mathrm{H}), 7.82(\mathrm{~d}, J=8.3 \mathrm{~Hz}$, $2 \mathrm{H}, \mathrm{Ar}-\mathrm{H}) ;{ }^{13} \mathrm{C} \mathrm{NMR}\left(75 \mathrm{MHz}, \mathrm{CDCl}_{3}\right): \delta=39.6,40.1,118.9,125.5,128.8,137.5,154.5$, 170.0.; $\mathrm{MS}\left(\mathrm{ESI}^{+}\right) \mathrm{m} / \mathrm{z}=209(\mathrm{M}+\mathrm{H})^{+}$; $\mathrm{HRMS}\left(\mathrm{ESI}^{+}\right)$calcd for $\mathrm{C}_{10} \mathrm{H}_{10} \mathrm{ClN}_{2} \mathrm{O}(\mathrm{M}+\mathrm{H})^{+}$ 209.0474, found 209.0476.

3-(Chloromethyl)-1-(3-nitrophenyl)-1 $H$-pyrazol-5(4H)-one (5b)

Pale yellow solid; Yield: 52\%; Mp 84-86 ${ }^{\circ} \mathrm{C}$; IR (KBr) 2924, 2854, 1736, 1532, 1352, 1139 , 1024, 739, $673 \mathrm{~cm}^{-1} ;{ }^{1} \mathrm{H}$ NMR (300 MHz, $\left.\mathrm{CDCl}_{3}\right): \delta=3.71\left(\mathrm{~s}, 2 \mathrm{H}, \mathrm{CH}_{2}\right), 4.44(\mathrm{~s}, 2 \mathrm{H}$, 
$\left.\mathrm{CH}_{2} \mathrm{Cl}\right), 7.55(\mathrm{t}, J=8.1,1 \mathrm{H}, \mathrm{Ar}-\mathrm{H}), 8.02(\mathrm{dd}, J=0.9,8.1 \mathrm{~Hz}, 1 \mathrm{H}, \mathrm{Ar}-\mathrm{H}), 8.27(\mathrm{dd}, J=0.9$, $8.3 \mathrm{~Hz}, 1 \mathrm{H}, \mathrm{Ar}-\mathrm{H}), 8.68(\mathrm{~s}, 1 \mathrm{H}, \mathrm{Ar}-\mathrm{H}) ;{ }^{13} \mathrm{C} \mathrm{NMR}\left(75 \mathrm{MHz}, \mathrm{CDCl}_{3}\right): \delta=40.3,58.8,69.5$, $116.5,118.6,125.0,129.8,134.5,138.8,156.9,170.3 . ; \mathrm{MS}_{\left(\mathrm{ESI}^{+}\right)} \mathrm{m} / \mathrm{z}=254(\mathrm{M}+\mathrm{H})^{+}$; HRMS (ESI ${ }^{+}$) calcd for $\mathrm{C}_{10} \mathrm{H}_{9} \mathrm{ClN}_{3} \mathrm{O}_{3}(\mathrm{M}+\mathrm{H})^{+} 254.0327$, found 254.0325 .

3-(Chloromethyl)-5-ethoxy-1-phenyl-1H-pyrazole (6a)

Yellow liquid; Yield: 5\%; IR (KBr) 2928, 1561, 1262, 1140, 1044, 758, $690 \mathrm{~cm}^{-1}$; ${ }^{1} \mathrm{H}$ NMR $\left(300 \mathrm{MHz}, \mathrm{CDCl}_{3}\right): \delta=1.45\left(\mathrm{t}, J=7.0 \mathrm{~Hz}, 3 \mathrm{H}, \mathrm{CH}_{3}\right), 4.18\left(\mathrm{q}, J=7.0 \mathrm{~Hz}, 2 \mathrm{H}, \mathrm{OCH}_{2}\right), 4.57$ (s, 2H, $\left.\mathrm{CH}_{2}\right), 5.75(\mathrm{~s}, 1 \mathrm{H}, \mathrm{CH}), 7.27$ (tt, $\left.J=1.1,7.5 \mathrm{~Hz}, 1 \mathrm{H}, \mathrm{Ar}-\mathrm{H}\right), 7.41(\mathrm{t}, J=7.5 \mathrm{~Hz}, 2 \mathrm{H}$, Ar-H), 7.69 (dd, $J=1.0,8.5 \mathrm{~Hz}, 2 \mathrm{H}, \mathrm{Ar}-\mathrm{H}) ;{ }^{13} \mathrm{C} \mathrm{NMR}\left(75 \mathrm{MHz}, \mathrm{CDCl}_{3}\right): \delta=4.5,39.8,68.0$,

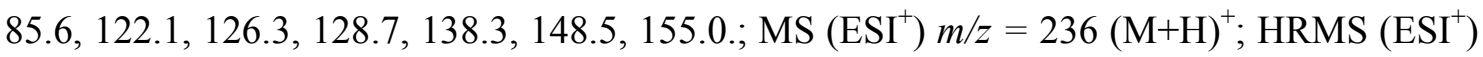
calcd for $\mathrm{C}_{12} \mathrm{H}_{13} \mathrm{ClN}_{2} \mathrm{ONa}(\mathrm{M}+\mathrm{Na})^{+} 259.0608$, found 259.0614 .

3-(Chloromethyl)-5-ethoxy-1-(3-nitrophenyl)-1H-pyrazole (6b)

Yellow liquid; Yield: 10\%; IR (KBr) 2986, 1527, 1351, 1263, 1008, 735, $681 \mathrm{~cm}^{-1} ;{ }^{1} \mathrm{H}$ NMR $\left(300 \mathrm{MHz}, \mathrm{CDCl}_{3}\right): \delta=1.52\left(\mathrm{t}, J=7.0 \mathrm{~Hz}, 3 \mathrm{H}, \mathrm{CH}_{3}\right), 4.26\left(\mathrm{q}, J=7.0 \mathrm{~Hz}, 2 \mathrm{H}, \mathrm{OCH}_{2}\right), 4.56$ (s, 2H, $\left.\mathrm{CH}_{2} \mathrm{Cl}\right), 5.79(\mathrm{~s}, 1 \mathrm{H}, \mathrm{CH}), 7.59$ (t, $\left.J=8.3 \mathrm{~Hz}, 1 \mathrm{H}, \mathrm{Ar}-\mathrm{H}\right), 8.13$ (ddd, $J=0.9,2.0$ and $8.3 \mathrm{~Hz}, 2 \mathrm{H}, \mathrm{Ar}-\mathrm{H}), 8.69(\mathrm{t}, J=2.0,1 \mathrm{H}, \mathrm{Ar}-\mathrm{H}) ;{ }^{13} \mathrm{C} \mathrm{NMR}\left(75 \mathrm{MHz}, \mathrm{CDCl}_{3}\right): \delta=14.5,39.4$, 68.6, 86.2, 116.2, 120.4, 126.7, 129.6, 139.4, 148.5, 149.8, 155.5.; MS $\left(\mathrm{ESI}^{+}\right) \mathrm{m} / z=282$ $(\mathrm{M}+\mathrm{H})^{+}$; HRMS $\left(\mathrm{ESI}^{+}\right)$calcd for $\mathrm{C}_{12} \mathrm{H}_{13} \mathrm{ClN}_{3} \mathrm{O}_{3}(\mathrm{M}+\mathrm{H})^{+}$282.0640, found 282.0643.

3-(Chloromethyl)-5-ethoxy-1-(4-fluorophenyl)-1H-pyrazole (6c)

Yellow liquid; Yield: 18\%; IR (KBr) 2923, 1515, 1224, 1095, 837, $771 \mathrm{~cm}^{-1}$; ${ }^{1} \mathrm{H}$ NMR (300 $\left.\mathrm{MHz}, \mathrm{CDCl}_{3}\right): \delta=1.52\left(\mathrm{t}, J=7.0 \mathrm{~Hz}, 3 \mathrm{H}, \mathrm{CH}_{3}\right), 4.26\left(\mathrm{t}, J=7.0 \mathrm{~Hz}, 2 \mathrm{H}, \mathrm{OCH}_{2}\right), 4.56(\mathrm{~s}, 2 \mathrm{H}$, $\left.\mathrm{CH}_{2} \mathrm{Cl}\right), 5.76(\mathrm{~s}, 1 \mathrm{H}, \mathrm{CH}), 7.58(\mathrm{t}, J=2.1 \mathrm{~Hz}, 1 \mathrm{H}, \mathrm{Ar}-\mathrm{H}), 8.13(\mathrm{ddd}, J=8.2,2.1$ and $0.7,2 \mathrm{H}$, $\operatorname{Ar}-\mathrm{H}), 8.69(\mathrm{t}, J=2.1 \mathrm{~Hz} 1 \mathrm{H}, \mathrm{Ar}-\mathrm{H}) ;{ }^{13} \mathrm{C} \mathrm{NMR}\left(75 \mathrm{MHz}, \mathrm{CDCl}_{3}\right): \delta=14.5,39.7,68.13$,

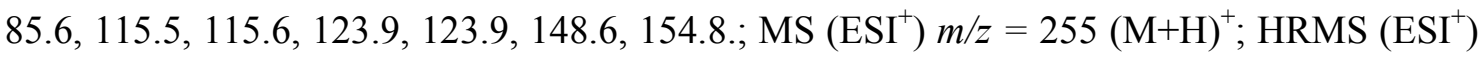
calcd for $\mathrm{C}_{12} \mathrm{H}_{13} \mathrm{ClFN}_{2} \mathrm{O}(\mathrm{M}+\mathrm{H})^{+}$255.0615, found 255.0700. 
3-(Chloromethyl)-5-ethoxy-1-p-tolyl-1H-pyrazole (6d)

Yellow liquid; Yield: 20\%; IR (KBr) 2926, 2854, 1663, 1525, 1323, 1216, 1112, 1033, 777 $\mathrm{cm}^{-1} ;{ }^{1} \mathrm{H}$ NMR $\left(300 \mathrm{MHz}, \mathrm{CDCl}_{3}\right): \delta=1.43\left(\mathrm{t}, J=7.0 \mathrm{~Hz}, 3 \mathrm{H}, \mathrm{CH}_{3}\right), 3.26\left(\mathrm{~s}, 2 \mathrm{H}, \mathrm{CH}_{3}\right), 4.16$ $\left(\mathrm{q}, J=7.0 \mathrm{~Hz}, 2 \mathrm{H}, \mathrm{OCH}_{2}\right), 4.56\left(\mathrm{~s}, 2 \mathrm{H}, \mathrm{CH}_{2} \mathrm{Cl}\right), 5.76(\mathrm{~s}, 1 \mathrm{H}, \mathrm{CH}), 7.21(\mathrm{~d}, J=8.8 \mathrm{~Hz}, 2 \mathrm{H}$,

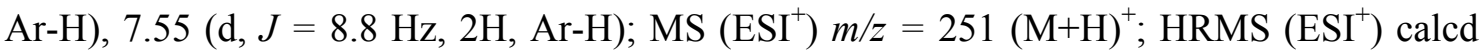
for $\mathrm{C}_{13} \mathrm{H}_{17} \mathrm{ClN}_{2} \mathrm{O}(\mathrm{M}+\mathrm{H})^{+} 251.0945$, found 251.0944 .

3-(Chloromethyl)-5-ethoxy-1-(4-nitrophenyl)-1H-pyrazole (6e)

Colorless liquid; Yield: 22\%; IR (KBr) 2983, 1597, 1508, 1335, 1112, 1038, 849, $750 \mathrm{~cm}^{-1}$; ${ }^{1} \mathrm{H}$ NMR $\left(300 \mathrm{MHz}, \mathrm{CDCl}_{3}\right): \delta=1.52\left(\mathrm{t}, J=6.9 \mathrm{~Hz}, 3 \mathrm{H}, \mathrm{CH}_{3}\right), 4.25(\mathrm{q}, J=6.9 \mathrm{~Hz}, 2 \mathrm{H}$, $\left.\mathrm{OCH}_{2}\right), 4.55\left(\mathrm{~s}, 2 \mathrm{H}, \mathrm{CH}_{2} \mathrm{Cl}\right), 5.80(\mathrm{~s}, 1 \mathrm{H}, \mathrm{CH}), 8.01(\mathrm{~d}, J=9.2 \mathrm{~Hz}, 2 \mathrm{H}, \mathrm{Ar}-\mathrm{H}), 8.29(\mathrm{~d}, J=$ $9.2 \mathrm{~Hz}, 2 \mathrm{H}, \mathrm{Ar}-\mathrm{H}) ;{ }^{13} \mathrm{C} \mathrm{NMR}\left(75 \mathrm{MHz}, \mathrm{CDCl}_{3}\right): \delta=14.5,39.3,68.7,86.6,120.8,124.6$, 143.5, 144.9, 150.4, 155.9.; $\mathrm{MS}\left(\mathrm{ESI}^{+}\right) m / z=282(\mathrm{M}+\mathrm{H})^{+}$.

3-(Methoxymethyl)-1-phenyl-1H-pyrazol-5(4H)-one (7a)

Pale yellow solid; Yield: 52\%; Mp 122-124 ${ }^{\circ} \mathrm{C}$; IR (KBr) 2925, 2821, 1737, 1553, 1396, $1111,1029,755,686 \mathrm{~cm}^{-1} ;{ }^{1} \mathrm{H}$ NMR $\left(300 \mathrm{MHz}, \mathrm{CDCl}_{3}\right): \delta=3.42\left(\mathrm{~s}, 3 \mathrm{H}, \mathrm{OCH}_{3}\right), 3.54(\mathrm{~s}, 2 \mathrm{H}$, $\left.\mathrm{CH}_{2}\right), 4.28\left(\mathrm{~s}, 2 \mathrm{H}, \mathrm{OCH}_{2}\right), 7.19(\mathrm{t}, J=7.4 \mathrm{~Hz}, 1 \mathrm{H}, \mathrm{Ar}-\mathrm{H}), 7.40(\mathrm{t}, J=7.7 \mathrm{~Hz}, 2 \mathrm{H}, \mathrm{Ar}-\mathrm{H})$, $7.85(\mathrm{~d}, J=7.7 \mathrm{~Hz}, 2 \mathrm{H}, \mathrm{Ar}-\mathrm{H}) ;{ }^{13} \mathrm{C} \mathrm{NMR}\left(75 \mathrm{MHz}, \mathrm{CDCl}_{3}\right): \delta=40.3,58.8,69.6,118.9$, 125.2, 128.8, 137.8.; MS $\left(\mathrm{ESI}^{+}\right) \mathrm{m} / z=205(\mathrm{M}+\mathrm{H})^{+}$; HRMS $\left(\mathrm{ESI}^{+}\right)$calcd for $\mathrm{C}_{11} \mathrm{H}_{13} \mathrm{~N}_{2} \mathrm{O}_{2}$ $(\mathrm{M}+\mathrm{H})^{+}$205.0970, found 205.0971.

3-(Ethoxymethyl)-1-phenyl-1 $H$-pyrazol-5(4H)-one (7b)

Yellow liquid; Yield: 45\%; IR (KBr) 2923, 2820, 1729, 1559, 1406, 1311, 1156, 1096, 1027 , 950, 812, 768, $641 \mathrm{~cm}^{-1} ;{ }^{1} \mathrm{H}$ NMR (300 MHz, $\left.\mathrm{CDCl}_{3}\right): \delta=1.25\left(\mathrm{t}, J=6.8 \mathrm{~Hz}, 3 \mathrm{H}, \mathrm{CH}_{3}\right)$, $3.55\left(\mathrm{~s}, 2 \mathrm{H}, \mathrm{CH}_{2}\right), 3.58$ (q, $\left.J=6.8 \mathrm{~Hz}, 2 \mathrm{H}, \mathrm{OCH}_{2}\right), 4.32\left(\mathrm{~s}, 2 \mathrm{H}, \mathrm{OCH}_{2}\right), 7.19$ (t, $J=7.5,1 \mathrm{H}$, Ar-H), 7.39 (t, $J=7.5 \mathrm{~Hz}, 2 \mathrm{H}, \mathrm{Ar}-\mathrm{H}), 7.85(\mathrm{t}, J=7.5 \mathrm{~Hz}, 2 \mathrm{H}, \mathrm{Ar}-\mathrm{H}) .{ }^{13} \mathrm{C}$ NMR $(75 \mathrm{MHz}$, 
$\left.\mathrm{CDCl}_{3}\right): \delta=15.0,40.4,66.6,67.7,118.9,125.2,128.8,137.8,156.9,170.5 . ; \mathrm{MS}\left(\mathrm{ESI}^{+}\right) \mathrm{m} / \mathrm{z}$ $=219(\mathrm{M}+\mathrm{H})^{+}$; HRMS $\left(\mathrm{ESI}^{+}\right)$calcd for $\mathrm{C}_{12} \mathrm{H}_{15} \mathrm{~N}_{2} \mathrm{O}_{2}(\mathrm{M}+\mathrm{H})^{+} 219.1128$, found 219.1131.

3-(Isopropoxymethyl)-1-phenyl-1H-pyrazol-5(4H)-one (7c)

Pale yellow solid; Yield: 39\%; Mp 123-125 ${ }^{\circ} \mathrm{C}$; IR (KBr) 2923, 2821, 1734, 1559, 1407, $1245,1156,1095,951,812 \mathrm{~cm}^{-1} ;{ }^{1} \mathrm{H}$ NMR $\left(300 \mathrm{MHz}, \mathrm{CDCl}_{3}\right): \delta=1.20\left(\mathrm{~s}, 3 \mathrm{H}, \mathrm{CH}_{3}\right), 1.23(\mathrm{~s}$, $\left.3 \mathrm{H}, \mathrm{CH}_{3}\right), 3.55\left(\mathrm{~s}, 2 \mathrm{H}, \mathrm{CH}_{2}\right), 3.65-3.74(\mathrm{~m}, 1 \mathrm{H} \mathrm{CH}), 4.32\left(\mathrm{~s}, 2 \mathrm{H}, \mathrm{CH}_{2}\right), 7.19(\mathrm{t}, J=7.5 \mathrm{~Hz}$, 1H, Ar-H), 7.34 (t, $J=7.5 \mathrm{~Hz}, 2 \mathrm{H}, \mathrm{Ar}-\mathrm{H}), 7.84(\mathrm{~d}, J=7.7 \mathrm{~Hz}, 2 \mathrm{H}, \mathrm{Ar}-\mathrm{H}) ;{ }^{13} \mathrm{C}$ NMR $(75$ $\left.\mathrm{MHz}, \mathrm{CDCl}_{3}\right): \delta=21.9,40.4,65.4,72.2,118.9,125.2,128.8,137.8,157.4,170.5 . ; \mathrm{MS}$ $\left(\mathrm{ESI}^{+}\right) m / z=233(\mathrm{M}+\mathrm{H})^{+}$; HRMS $\left(\mathrm{ESI}^{+}\right)$calcd for $\mathrm{C}_{13} \mathrm{H}_{17} \mathrm{~N}_{2} \mathrm{O}_{2}(\mathrm{M}+\mathrm{H})^{+}$233.1284, found 233.1287.

3-(Isobutoxymethyl)-1-phenyl-1H-pyrazol-5(4H)-one (7d)

Pale yellow solid; Yield: 36\%; Mp 78-90 ${ }^{\circ} \mathrm{C}$; IR (KBr) 2959, 2873, 1727, 1551, 1258, 1090, 755, $682 \mathrm{~cm}^{-1} ;{ }^{1} \mathrm{H}$ NMR $\left(300 \mathrm{MHz}, \mathrm{CDCl}_{3}\right): \delta=0.92\left(\mathrm{~s}, 3 \mathrm{H}, \mathrm{CH}_{3}\right), 0.94\left(\mathrm{~s}, 3 \mathrm{H}, \mathrm{CH}_{3}\right), 1.85-$ $1.93(\mathrm{~m}, 1 \mathrm{H}, \mathrm{CH}) ; 3.27\left(\mathrm{~d}, J=6.6 \mathrm{~Hz}, 2 \mathrm{H}, \mathrm{CH}_{2}\right), 3.55\left(\mathrm{~s}, 2 \mathrm{H}, \mathrm{CH}_{2}\right), 4.31\left(\mathrm{~s}, 2 \mathrm{H}, \mathrm{OCH}_{2}\right), 7.19$ $(\mathrm{t}, J=7.3 \mathrm{~Hz}, 1 \mathrm{H}, \mathrm{Ar}-\mathrm{H}), 7.40(\mathrm{t}, J=7.5 \mathrm{~Hz}, 2 \mathrm{H}, \mathrm{Ar}-\mathrm{H}), 7.85(\mathrm{~d}, J=8.6 \mathrm{~Hz}, 2 \mathrm{H}, \mathrm{Ar}-\mathrm{H}) ;{ }^{13} \mathrm{C}$ $\operatorname{NMR}\left(75 \mathrm{MHz}, \mathrm{CDCl}_{3}\right): \delta=19.2,28.4,40.4,68.1,78.0,118.9,125.2,128.7,137.8,157.1$, 170.1.; $\mathrm{MS}\left(\mathrm{ESI}^{+}\right) \mathrm{m} / \mathrm{z}=247(\mathrm{M}+\mathrm{H})^{+}$; HRMS $\left(\mathrm{ESI}^{+}\right)$calcd for $\mathrm{C}_{14} \mathrm{H}_{19} \mathrm{~N}_{2} \mathrm{O}_{2}(\mathrm{M}+\mathrm{H})^{+}$ 247.1441, found 247.1443.

3-(Butoxymethyl)-1-phenyl-1H-pyrazol-5(4H)-one (7e)

Pale yellow solid; Yield: 35\%; Mp 76-78 ${ }^{\circ} \mathrm{C}$; IR (KBr) 2957, 2872, 1746, 1527, 1351, 1169 , 1096, 758, $696 \mathrm{~cm}^{-1} ;{ }^{1} \mathrm{H}$ NMR (300 MHz, $\left.\mathrm{CDCl}_{3}\right): \delta=0.93\left(\mathrm{t}, J=7.3,3 \mathrm{H}, \mathrm{CH}_{3}\right), 1.35-1.42$ $\left(\mathrm{m}, 2 \mathrm{H}, \mathrm{CH}_{2}\right), 1.56-1.62\left(\mathrm{~m}, 2 \mathrm{H}, \mathrm{CH}_{2}\right), 3.50\left(\mathrm{t}, J=6.6 \mathrm{~Hz}, 2 \mathrm{H}, \mathrm{CH}_{2}\right), 3.54\left(\mathrm{~s}, 2 \mathrm{H}, \mathrm{CH}_{2}\right), 4.31$ $\left(\mathrm{s}, 2 \mathrm{H}, \mathrm{OCH}_{2}\right), 7.19(\mathrm{t}, J=7.3 \mathrm{~Hz}, 1 \mathrm{H}, \mathrm{Ar}-\mathrm{H}), 7.39$ (t, $\left.J=8.2 \mathrm{~Hz}, 2 \mathrm{H}, \mathrm{Ar}-\mathrm{H}\right), 7.62(\mathrm{~d}, J=7.6$ $\mathrm{Hz}, 2 \mathrm{H}, \mathrm{Ar}-\mathrm{H}) ;{ }^{13} \mathrm{C}$ NMR $\left(75 \mathrm{MHz}, \mathrm{CDCl}_{3}\right): \delta=13.8,19.2,31.5,40.4,67.9,71.1,118.9$, 
125.2, 128.7, 137.8, 157.0, 170.5; $\mathrm{MS}\left(\mathrm{ESI}^{+}\right) m / z=247(\mathrm{M}+\mathrm{H})^{+}$; HRMS $\left(\mathrm{ESI}^{+}\right)$calcd for $\mathrm{C}_{14} \mathrm{H}_{19} \mathrm{~N}_{2} \mathrm{O}_{2}(\mathrm{M}+\mathrm{H})^{+}$247.1441, found 247.1444.

1-(4-Fluorophenyl)-3-(methoxymethyl)-1H-pyrazol-5(4H)-one (7f)

Colorless solid; Yield: 56\%; Mp 110-112 ${ }^{\circ} \mathrm{C}$; IR (KBr) 2915, 1743, 1565, 1244, 1028, 836, $761,647 \mathrm{~cm}^{-1} ;{ }^{1} \mathrm{H}$ NMR $\left(300 \mathrm{MHz}, \mathrm{CDCl}_{3}\right): \delta=3.43\left(\mathrm{~s}, 3 \mathrm{H}, \mathrm{OCH}_{3}\right), 3.54\left(\mathrm{~s}, 2 \mathrm{H}, \mathrm{CH}_{2}\right), 4.27$ $\left(\mathrm{s}, 2 \mathrm{H}, \mathrm{CH}_{2}\right), 7.08(\mathrm{t}, J=8.5 \mathrm{~Hz}, 2 \mathrm{H}, \mathrm{Ar}-\mathrm{H}), 7.79-7.85(\mathrm{~m}, 2 \mathrm{H}, \mathrm{Ar}-\mathrm{H}) ;{ }^{13} \mathrm{C} \mathrm{NMR}(75 \mathrm{MHz}$, $\left.\mathrm{CDCl}_{3}\right): \delta=40.2,58.9,69.6,115.4,115.7,120.7,156.7,158.4,170.3 . ; \mathrm{MS}\left(\mathrm{ESI}^{+}\right) \mathrm{m} / z=223$ $(\mathrm{M}+\mathrm{H})^{+}$; HRMS $\left(\mathrm{ESI}^{+}\right)$calcd for $\mathrm{C}_{11} \mathrm{H}_{12} \mathrm{FN}_{2} \mathrm{O}_{2}(\mathrm{M}+\mathrm{H})^{+}$223.0877, found 223.0876.

1-(4-Bromophenyl)-3-(methoxymethyl)-1H-pyrazol-5(4H)-one (7g)

Pale yellow solid; Yield: 54\%; Mp 115-117 ${ }^{\circ} \mathrm{C}$; IR (KBr) 2923, 2820, 1729, 1559, 1406, 1245, 1096, 812, $768 \mathrm{~cm}^{-1} ;{ }^{1} \mathrm{H}$ NMR $\left(300 \mathrm{MHz} \mathrm{CDCl}_{3}\right): \delta=3.43\left(\mathrm{~s}, 3 \mathrm{H}, \mathrm{OCH}_{3}\right), 3.54(\mathrm{~s}, 2 \mathrm{H}$, $\left.\mathrm{CH}_{2}\right), 4.27\left(\mathrm{~s}, 2 \mathrm{H}, \mathrm{CH}_{2}\right), 7.50(\mathrm{~d}, J=8.8 \mathrm{~Hz}, 2 \mathrm{H}, \mathrm{Ar}-\mathrm{H}), 7.78(\mathrm{~d}, J=8.8,2 \mathrm{H}, \mathrm{Ar}-\mathrm{H}) .{ }^{13} \mathrm{C}$ NMR (75 MHz, $\left.\mathrm{CDCl}_{3}\right): \delta=40.3,58.9,69.5,118.0,120.2,131.8,136.9,156.9,170.3 . ; \mathrm{MS}$ $\left(\mathrm{ESI}^{+}\right) m / z=283(\mathrm{M}+\mathrm{H})^{+} ; \mathrm{HRMS}\left(\mathrm{ESI}^{+}\right)$calcd for $\mathrm{C}_{11} \mathrm{H}_{12} \mathrm{BrN}_{2} \mathrm{O}_{2}(\mathrm{M}+\mathrm{H})^{+}$283.0076, found 283.0074 .

3-(Methoxymethyl)-1-(4-methoxyphenyl)-1H-pyrazol-5(4H)-one (7h)

Pale yellow solid; Yield: 50\%; Mp 120-122 ${ }^{\circ} \mathrm{C}$; IR (KBr) 2957, 1738, 1561, 1405, 1250, 1034, 822, 764, $644 \mathrm{~cm}^{-1} ;{ }^{1} \mathrm{H}$ NMR $\left(300 \mathrm{MHz}, \mathrm{CDCl}_{3}\right): \delta=3.42\left(\mathrm{~s}, 3 \mathrm{H}, \mathrm{OCH}_{3}\right), 3.52(\mathrm{~s}, 2 \mathrm{H}$, $\left.\mathrm{CH}_{2}\right), 3.81\left(\mathrm{~s}, 3 \mathrm{H}, \mathrm{OCH}_{3}\right), 4.27\left(\mathrm{~s}, 2 \mathrm{H}, \mathrm{CH}_{2}\right), 6.92(\mathrm{~d}, J=9.2 \mathrm{~Hz}, 2 \mathrm{H}, \mathrm{Ar}-\mathrm{H}), 7.71(\mathrm{~d}, J=9.2$ $\mathrm{Hz}, 2 \mathrm{H}, \mathrm{Ar}-\mathrm{H}) ;{ }^{13} \mathrm{C} \mathrm{NMR}\left(75 \mathrm{MHz}, \mathrm{CDCl}_{3}\right): \delta=40.1,55.4,58.8,69.6,113.9,120.9,131.1$, 156.3, 157.1, 170.2.; $\mathrm{MS}\left(\mathrm{ESI}^{+}\right) \mathrm{m} / z=235(\mathrm{M}+\mathrm{H})^{+}$; HRMS $\left(\mathrm{ESI}^{+}\right)$calcd for $\mathrm{C}_{12} \mathrm{H}_{15} \mathrm{~N}_{2} \mathrm{O}_{3}$ $(\mathrm{M}+\mathrm{H})^{+}$235.1075, found 235.1077.

3-(Methoxymethyl)-1-p-tolyl-1H-pyrazol-5(4H)-one (7i)

Colorless solid; Yield: 51\%; Mp 112-114 ${ }^{\circ} \mathrm{C}$; IR (KBr) 2925, 2818, 1741, 1534, 1403, 1273 , 1093, 817, $770 \mathrm{~cm}^{-1} ;{ }^{1} \mathrm{H}$ NMR $\left(300 \mathrm{MHz}, \mathrm{CDCl}_{3}\right): \delta=2.34\left(\mathrm{~s}, 3 \mathrm{H}, \mathrm{CH}_{3}\right), 3.42\left(\mathrm{~s}, 3 \mathrm{H}, \mathrm{OCH}_{3}\right)$ 
$3.52\left(\mathrm{~s}, 2 \mathrm{H}, \mathrm{CH}_{2}\right) 4.27\left(\mathrm{~s}, 2 \mathrm{H}, \mathrm{CH}_{2}\right) 7.20(\mathrm{~d}, J=8.2 \mathrm{~Hz}, 2 \mathrm{H}, \mathrm{Ar}-\mathrm{H}) 7.70(\mathrm{~d}, J=8.5 \mathrm{~Hz}, 2 \mathrm{H}$, $\mathrm{Ar}-\mathrm{H}) ;{ }^{13} \mathrm{C} \mathrm{NMR}\left(75 \mathrm{MHz}, \mathrm{CDCl}_{3}\right): \delta=40.2,53.4,58.8,69.6,115.4,115.6,120.7,120.7$, 156.6, 170.2.; $\mathrm{MS}\left(\mathrm{ESI}^{+}\right) m / z=219(\mathrm{M}+\mathrm{H})^{+}$.

1-(3-Chlorophenyl)-3-(methoxymethyl)-1H-pyrazol-5(4H)-one (7j)

Pale yellow solid; Yield: 55\%; Mp 113-115 ${ }^{\circ} \mathrm{C}$; IR (KBr) 2914, 1741, 1591, 1401, 1244, 1097, 787, $682 \mathrm{~cm}^{-1} ;{ }^{1} \mathrm{H}$ NMR $\left(300 \mathrm{MHz}, \mathrm{CDCl}_{3}\right): \delta=3.43\left(\mathrm{~s}, 3 \mathrm{H}, \mathrm{OCH}_{3}\right), 3.55\left(\mathrm{~s}, 2 \mathrm{H}, \mathrm{CH}_{2}\right)$, $4.28\left(\mathrm{~s}, 2 \mathrm{H}, \mathrm{CH}_{2}\right), 7.16$ (ddd, $J=0.9,1.1$, and $\left.8.9 \mathrm{~Hz}, 1 \mathrm{H}, \mathrm{Ar}-\mathrm{H}\right), 7.31(\mathrm{t}, J=8.0 \mathrm{~Hz}, 1 \mathrm{H}, \mathrm{Ar}-$ $\mathrm{H}), 7.82(\mathrm{ddd}, J=0.9,1.1$, and $8.2 \mathrm{~Hz}, 1 \mathrm{H}, \mathrm{Ar}-\mathrm{H}), 7.91(\mathrm{t}, J=1.1 \mathrm{~Hz}, 1 \mathrm{H}, \mathrm{Ar}-\mathrm{H}) ;{ }^{13} \mathrm{C} \mathrm{NMR}$ $\left(75 \mathrm{MHz}, \mathrm{CDCl}_{3}\right): \delta=40.3,58.8,69.5,116.5,118.6,125.0,129.8,134.5,138.8,156.9$, 170.3.; $\mathrm{MS}\left(\mathrm{ESI}^{+}\right) \mathrm{m} / z=239(\mathrm{M}+\mathrm{H})^{+} ; \operatorname{HRMS}\left(\mathrm{ESI}^{+}\right)$calcd for $\mathrm{C}_{11} \mathrm{H}_{12} \mathrm{ClN}_{2} \mathrm{O}_{2}(\mathrm{M}+\mathrm{H})^{+}$ 239.0580, found 239.0581 .

3-(Methoxymethyl)-1-(3-nitrophenyl)-1H-pyrazol-5(4H)-one (7k)

Pale yellow solid; Yield: 58\%; Mp 96-98 ${ }^{\circ} \mathrm{C}$; IR (KBr) 2924, 1621, 1530, 1350, 1273, 1097, 894, $737 \mathrm{~cm}^{-1} ;{ }^{1} \mathrm{H}$ NMR $\left(300 \mathrm{MHz}, \mathrm{CDCl}_{3}\right): \delta=3.45\left(\mathrm{~s}, 3 \mathrm{H}, \mathrm{OCH}_{3}\right), 3.61\left(\mathrm{~s}, 2 \mathrm{H}, \mathrm{CH}_{2}\right), 4.32$ $\left(s, 2 \mathrm{H}, \mathrm{CH}_{2}\right), 7.56(\mathrm{t}, J=8.2 \mathrm{~Hz}, 1 \mathrm{H}, \mathrm{Ar}-\mathrm{H}), 8.04(\mathrm{ddd}, J=0.9,2.1$ and $8.2 \mathrm{~Hz}, 1 \mathrm{H}, \mathrm{Ar}-\mathrm{H})$, $8.34(\mathrm{dd}, J=1.0,8.2,1 \mathrm{H}, \mathrm{Ar}-\mathrm{H}), 8.74(\mathrm{t}, J=2.1,1 \mathrm{H}, \mathrm{Ar}-\mathrm{H}) ;{ }^{13} \mathrm{C} \mathrm{NMR}\left(75 \mathrm{MHz}, \mathrm{CDCl}_{3}\right): \delta$ $\left.=40.4,58.9,69.4,113.2,116.6,119.4,123.7,127.1,129.7,157.6,170.5 . ; \mathrm{MS}_{(\mathrm{ESI}}^{+}\right) \mathrm{m} / \mathrm{z}=$ $272(\mathrm{M}+\mathrm{Na})^{+}$; HRMS $\left(\mathrm{ESI}^{+}\right)$calcd for $\mathrm{C}_{11} \mathrm{H}_{11} \mathrm{ClN}_{3} \mathrm{O}_{4} \mathrm{Na}(\mathrm{M}+\mathrm{Na})^{+} 272.0641$, found 272.0642. 3-(Ethoxymethyl)-1-p-tolyl-1 $H$-pyrazol-5(4H)-one (7l)

Yellow liquid; Yield: 45\%; IR (KBr) 2925, 2861, 1719, 1517, 1104, 1022, 818, $764 \mathrm{~cm}^{-1}$; ${ }^{1} \mathrm{H}$ $\operatorname{NMR}\left(300 \mathrm{MHz}, \mathrm{CDCl}_{3}\right): \delta=1.25\left(\mathrm{t}, J=7.0,3 \mathrm{H}, \mathrm{CH}_{3}\right), 2.34\left(\mathrm{~s}, 3 \mathrm{H}, \mathrm{CH}_{3}\right) 3.53\left(\mathrm{~s}, 2 \mathrm{H}, \mathrm{CH}_{2}\right)$, $3.57\left(\mathrm{q}, J=7.0 \mathrm{~Hz}, 2 \mathrm{H}, \mathrm{OCH}_{2}\right), 4.30\left(\mathrm{~s}, 2 \mathrm{H}, \mathrm{CH}_{2}\right), 7.19$ (d, $\left.J=8.3 \mathrm{~Hz}, 2 \mathrm{H}, \mathrm{Ar}-\mathrm{H}\right), 7.70(\mathrm{~d}, J$ $=8.5 \mathrm{~Hz}, 2 \mathrm{H}, \mathrm{Ar}-\mathrm{H}) ;{ }^{13} \mathrm{C} \mathrm{NMR}\left(75 \mathrm{MHz}, \mathrm{CDCl}_{3}\right): \delta=15.0,20.9,40.3,66.6,67.7,119.0$, 129.3, 134.9, 135.3, 156.7, 170.4.; MS $\left(\mathrm{ESI}^{+}\right) \mathrm{m} / \mathrm{z}=233(\mathrm{M}+\mathrm{H})^{+}$; HRMS $\left(\mathrm{ESI}^{+}\right)$calcd for $\mathrm{C}_{13} \mathrm{H}_{17} \mathrm{~N}_{2} \mathrm{O}_{2}(\mathrm{M}+\mathrm{H})^{+} 233.1284$, found 233.1281. 
1-(3-Chlorophenyl)-3-(ethoxymethyl)-1H-pyrazol-5(4H)-one (7m)

Yellow liquid; Yield: 39\%; IR (KBr) 2926, 1737, 1593, 1347, 1255, 1098, 875, $779 \mathrm{~cm}^{-1}$; ${ }^{1} \mathrm{H}$ $\operatorname{NMR}\left(300 \mathrm{MHz}, \mathrm{CDCl}_{3}\right): \delta=1.25\left(\mathrm{t}, J=7.0 \mathrm{~Hz}, 3 \mathrm{H}, \mathrm{CH}_{3}\right), 3.56\left(\mathrm{~s}, 2 \mathrm{H}, \mathrm{CH}_{2}\right), 3.59(\mathrm{q}, J=$ $\left.7.0 \mathrm{~Hz}, 2 \mathrm{H}, \mathrm{OCH}_{2}\right), 4.32\left(\mathrm{~s}, 2 \mathrm{H}, \mathrm{CH}_{2}\right), 7.16(\mathrm{ddd}, J=0.9,1.1$ and $7.9 \mathrm{~Hz}, 1 \mathrm{H}, \mathrm{Ar}-\mathrm{H}), 7.31$ (t, $J=8.1 \mathrm{~Hz}, 1 \mathrm{H}, \mathrm{Ar}-\mathrm{H}), 7.82(\mathrm{ddd}, J=0.9,1.1$ and $8.2 \mathrm{~Hz}, 1 \mathrm{H}, \mathrm{Ar}-\mathrm{H}), 7.91$ (t, $J=1.1 \mathrm{~Hz}, 1 \mathrm{H}$, $\mathrm{Ar}-\mathrm{H}) ;{ }^{13} \mathrm{C} \mathrm{NMR}\left(75 \mathrm{MHz}, \mathrm{CDCl}_{3}\right): \delta=15.0,40.4,66.8,67.7,116.5,118.7,125.1,129.8$, 134.5, 138.9, 157.3, 170.4.; $\mathrm{MS}\left(\mathrm{ESI}^{+}\right) \mathrm{m} / \mathrm{z}=253(\mathrm{M}+\mathrm{Na})^{+}$; HRMS (ESI $\left.{ }^{+}\right)$calcd for $\mathrm{C}_{12} \mathrm{H}_{13} \mathrm{ClN}_{2} \mathrm{O}_{2} \mathrm{Na}[\mathrm{M}+\mathrm{Na}]^{+}$253.0738, found 253.0741.

3-(Ethoxymethyl)-1-(3-nitrophenyl)-1H-pyrazol-5(4H)-one (7n)

Yellow liquid; Yield: 51\%; IR (KBr) 2925, 1726, 1531, 1349, 1250, 1154, 1096, 834, 737 $\mathrm{cm}^{-1} ;{ }^{1} \mathrm{H} \mathrm{NMR}\left(300 \mathrm{MHz}, \mathrm{CDCl}_{3}\right): \delta=1.24\left(\mathrm{t}, J=7.0 \mathrm{~Hz}, 3 \mathrm{H}, \mathrm{CH}_{3}\right), 3.54\left(\mathrm{~s}, 2 \mathrm{H}, \mathrm{CH}_{2}\right), 3.58$ $\left(\mathrm{q}, J=7.0 \mathrm{~Hz}, 2 \mathrm{H}, \mathrm{CH}_{2}\right), 4.31\left(\mathrm{~s}, 2 \mathrm{H}, \mathrm{CH}_{2}\right), 7.19(\mathrm{t}, J=7.5,1 \mathrm{H}, \mathrm{Ar}-\mathrm{H}), 7.39$ (t, $J=7.5 \mathrm{~Hz}$, 2H, Ar-H), $7.84(\mathrm{dd}, J=0.9,8.5 \mathrm{~Hz}, 2 \mathrm{H}, \mathrm{Ar}-\mathrm{H}) ;{ }^{13} \mathrm{C} \mathrm{NMR}\left(75 \mathrm{MHz}, \mathrm{CDCl}_{3}\right): \delta=15.0,17.0$, 43.1, 66.8, 67.5, 113.1, 119.1, 119.4, 123.6, 129.6, 138.9, 148.4, 157.2, 170.6.; MS (ESI $\left.{ }^{+}\right)$ $m / z=264(\mathrm{M}+\mathrm{H})^{+} ;$HRMS $\left(\mathrm{ESI}^{+}\right)$calcd for $\mathrm{C}_{12} \mathrm{H}_{13} \mathrm{~N}_{3} \mathrm{O}_{4}(\mathrm{M}+\mathrm{H})^{+}$264.0988, found 264.0979. 1-(3-Chlorophenyl)-3-(isopropoxymethyl)-1H-pyrazol-5(4H)-one (7o)

Yellow solid; Yield: 45\%; Mp 102-104 ${ }^{\circ} \mathrm{C} ;{ }^{1} \mathrm{H}$ NMR $\left(300 \mathrm{MHz}, \mathrm{CDCl}_{3}\right): \delta=1.21(\mathrm{~s}, 3 \mathrm{H}$, $\left.\mathrm{CH}_{3}\right), 1.22\left(\mathrm{~s}, 3 \mathrm{H}, \mathrm{CH}_{3}\right) 3.56\left(\mathrm{~s}, 2 \mathrm{H}, \mathrm{CH}_{2}\right), 3.66-3.74(\mathrm{~m}, 1 \mathrm{H}, \mathrm{CH}), 4.32\left(\mathrm{~s}, 2 \mathrm{H}, \mathrm{CH}_{2}\right), 7.16$ (ddd, $J=0.8,1.1$ and $7.9 \mathrm{~Hz}, 1 \mathrm{H}, \mathrm{Ar}-\mathrm{H}), 7.31(\mathrm{t}, J=8.0 \mathrm{~Hz}, 1 \mathrm{H}, \mathrm{Ar}-\mathrm{H}), 7.82$ (ddd, $J=0.8$, 1.1 and 8.2, $1 \mathrm{H}, \mathrm{Ar}-\mathrm{H}), 7.91(\mathrm{t}, J=1.1 \mathrm{~Hz}, 1 \mathrm{H}, \mathrm{Ar}-\mathrm{H}) ;{ }^{13} \mathrm{C} \mathrm{NMR}\left(75 \mathrm{MHz}, \mathrm{CDCl}_{3}\right): \delta=21.9$, $39.7,40.0,40.5,65.3,72.3,116.5,116.5,118.7,125.0,129.8,157.8,170.5 . ; \mathrm{MS}_{\left(\mathrm{ESI}^{+}\right)} \mathrm{m} / \mathrm{z}=$ $266(\mathrm{M}+\mathrm{H})^{+}$; HRMS $\left(\mathrm{ESI}^{+}\right)$calcd for $\mathrm{C}_{13} \mathrm{H}_{15} \mathrm{ClN}_{2} \mathrm{O}_{2} \mathrm{Na}[\mathrm{M}+\mathrm{Na}]^{+}$289.0714, found 289.0712. 5-Ethoxy-3-(methoxymethyl)-1-phenyl-1H-pyrazole (8a)

Yellow liquid; Yield: 15\%; IR (KBr) 2925, 2854, 1509, 1456, 1266, 1096, 759, $693 \mathrm{~cm}^{-1} ;{ }^{1} \mathrm{H}$ $\operatorname{NMR}\left(300 \mathrm{MHz}, \mathrm{CDCl}_{3}\right): \delta=1.45\left(\mathrm{t}, J=7.0 \mathrm{~Hz}, 3 \mathrm{H}, \mathrm{CH}_{3}\right), 4.18\left(\mathrm{q}, J=7.0 \mathrm{~Hz}, 2 \mathrm{H}, \mathrm{OCH}_{2}\right)$, 
$4.57\left(\mathrm{~s}, 2 \mathrm{H}, \mathrm{CH}_{2}\right), 5.75(\mathrm{~s}, 1 \mathrm{H}, \mathrm{CH}), 7.27$ (t, $\left.J=7.4 \mathrm{~Hz}, 1 \mathrm{H}, \mathrm{Ar}-\mathrm{H}\right), 7.41(\mathrm{t}, J=7.4 \mathrm{~Hz}, 2 \mathrm{H}$, $\operatorname{Ar}-\mathrm{H}), 7.69(\mathrm{~d}, J=8.5 \mathrm{~Hz}, 2 \mathrm{H}, \mathrm{Ar}-\mathrm{H}) ;{ }^{13} \mathrm{C} \mathrm{NMR}\left(75 \mathrm{MHz}, \mathrm{CDCl}_{3}\right): \delta=14.5,58.1,67.8$, 69.0, 85.3, 122.0, 126.0, 128.6, 138.5, 149.4, 154.8.; MS $\left(\mathrm{ESI}^{+}\right) \mathrm{m} / z=233(\mathrm{M}+\mathrm{H})^{+}$; HRMS $\left(\mathrm{ESI}^{+}\right)$calcd for $\mathrm{C}_{13} \mathrm{H}_{17} \mathrm{~N}_{2} \mathrm{O}_{2}(\mathrm{M}+\mathrm{H})^{+}$233.1284, found 233.1289.

5-Ethoxy-3-(ethoxymethyl)-1-phenyl-1H-pyrazole (8b).

Yellow liquid; Yield: $18 \%$; ${ }^{1} \mathrm{H}$ NMR (300 MHz, $\left.\mathrm{CDCl} 3\right): \delta=1.25\left(\mathrm{t}, J=7.0 \mathrm{~Hz}, 1 \mathrm{H}, \mathrm{CH}_{3}\right)$, $1.43\left(\mathrm{t}, J=7.0 \mathrm{~Hz}, 3 \mathrm{H}, \mathrm{CH}_{3}\right), 3.60\left(\mathrm{q}, J=7.0 \mathrm{~Hz}, 2 \mathrm{H}, \mathrm{OCH}_{2}\right), 4.17$ (q, $J=7.0 \mathrm{~Hz}, 2 \mathrm{H}$, $\left.\mathrm{OCH}_{2}\right), 4.48\left(\mathrm{~s}, 2 \mathrm{H}, \mathrm{CH}_{2}\right), 5.72(\mathrm{~s}, 1 \mathrm{H}, \mathrm{CH}), 7.24(\mathrm{t}, J=7.4 \mathrm{~Hz}, 1 \mathrm{H}, \mathrm{Ar}-\mathrm{H}), 7.40(\mathrm{t}, J=7.4$ $\mathrm{Hz}, 2 \mathrm{H}, \mathrm{Ar}-\mathrm{H}), 7.70(\mathrm{dd}, J=8.8,0.7 \mathrm{~Hz}, 2 \mathrm{H}, \mathrm{Ar}-\mathrm{H}) ;{ }^{13} \mathrm{C} \mathrm{NMR}\left(75 \mathrm{MHz}, \mathrm{CDCl}_{3}\right): \delta=14.5$, $15.1,65.8,67.1,67.1,67.8,85.4 .122 .0,126.0,128.6,138.6,149.8,154.9 . ; \mathrm{MS}_{\left(\mathrm{ESI}^{+}\right)} \mathrm{m} / \mathrm{z}=$ $247(\mathrm{M}+\mathrm{H})^{+}$.

5-Ethoxy-1-(4-fluorophenyl)-3-(methoxymethyl)-1H-pyrazole (8c)

Yellow liquid; Yield: 14\%; IR (KBr) 2923, 2852, 1515, 1466, 1230, 1043, 836, $727 \mathrm{~cm}^{-1}$; ${ }^{1} \mathrm{H}$ $\operatorname{NMR}\left(300 \mathrm{MHz}, \mathrm{CDCl}_{3}\right): \delta=1.43\left(\mathrm{t}, J=7.0 \mathrm{~Hz}, 3 \mathrm{H}, \mathrm{CH}_{3}\right), 3.42\left(\mathrm{~s}, 3 \mathrm{H}, \mathrm{CH}_{3}\right), 4.16(\mathrm{q}, J=$ $\left.7.0 \mathrm{~Hz}, 2 \mathrm{H}, \mathrm{OCH}_{2}\right), 4.42\left(\mathrm{~s}, 2 \mathrm{H}, \mathrm{CH}_{2}\right), 5.70(\mathrm{~s}, 1 \mathrm{H}, \mathrm{CH}), 7.09$ (t, $\left.J=8.8 \mathrm{~Hz}, 2 \mathrm{H}, \mathrm{Ar}-\mathrm{H}\right), 7.65-$ $7.68(\mathrm{~m}, 2 \mathrm{H}, \mathrm{Ar}-\mathrm{H}) ; \mathrm{MS}\left(\mathrm{ESI}^{+}\right) \mathrm{m} / z=251(\mathrm{M}+\mathrm{H})^{+}$; HRMS $\left(\mathrm{ESI}^{+}\right)$calcd for $\mathrm{C}_{13} \mathrm{H}_{15} \mathrm{FN}_{2} \mathrm{O}_{2} \mathrm{Na}$ $[\mathrm{M}+\mathrm{Na}]^{+}$273.1009, found 273.1010.

1-(4-Bromophenyl)-5-ethoxy-3-(methoxymethyl)-1H-pyrazole (8d)

Yellow liquid; Yield: 18\%; IR (KBr) 2924, 2853, 1505, 1263, 1096, 827, $754 \mathrm{~cm}^{-1}$; ${ }^{1} \mathrm{H}$ NMR $\left(300 \mathrm{MHz}, \mathrm{CDCl}_{3}\right): \delta=1.44\left(\mathrm{dt}, J=1.5,7.0 \mathrm{~Hz}, 3 \mathrm{H}, \mathrm{CH}_{3}\right), 3.42\left(\mathrm{~s}, 3 \mathrm{H}, \mathrm{OCH}_{3}\right), 4.14$ (dq, $J=$ 1.5, 7.0 Hz, 2H, $\left.\mathrm{OCH}_{2}\right), 4.42\left(\mathrm{~s}, 2 \mathrm{H}, \mathrm{CH}_{2}\right), 5.70(\mathrm{~s}, 1 \mathrm{H}, \mathrm{CH}), 7.52(\mathrm{dd}, J=1.0,8.8 \mathrm{~Hz}, 2 \mathrm{H}$, Ar-H), $7.63(\mathrm{~d}, J=8.8 \mathrm{~Hz}, 2 \mathrm{H}, \mathrm{Ar}-\mathrm{H}) ; \mathrm{MS}\left(\mathrm{ESI}^{+}\right) \mathrm{m} / z=333(\mathrm{M}+\mathrm{Na})^{+}$; HRMS (ESI $\left.{ }^{+}\right)$calcd for $\mathrm{C}_{13} \mathrm{H}_{15} \mathrm{BrN}_{2} \mathrm{O}_{2} \mathrm{Na}(\mathrm{M}+\mathrm{Na})^{+} 333.0209$, found 333.0205. 
3-(Chloromethyl)-5-ethoxy-1-p-tolyl-1H-pyrazole (8e)

Yellow liquid; Yield: 12\%; IR (KBr) 2923, 1519, 1467, 1265, 1093, 818, $753 \mathrm{~cm}^{-1}$; ${ }^{1} \mathrm{H}$ NMR $\left(300 \mathrm{MHz}, \mathrm{CDCl}_{3}\right): \delta=1.44\left(\mathrm{dt}, J=1.5,7.0 \mathrm{~Hz}, 3 \mathrm{H}, \mathrm{CH}_{3}\right), 3.42\left(\mathrm{~s}, 3 \mathrm{H}, \mathrm{CH}_{3}\right), 4.14(\mathrm{dq}, J=$ 7.0, 1.5 Hz, 2H, $\left.\mathrm{OCH}_{2}\right), 4.42\left(\mathrm{~s}, 2 \mathrm{H}, \mathrm{CH}_{2}\right), 5.70(\mathrm{~s}, 1 \mathrm{H}, \mathrm{CH}), 7.52(\mathrm{dd}, J=8.8,1.0 \mathrm{~Hz}, 2 \mathrm{H}$, Ar-H), $7.63(\mathrm{td}, J=8.8,2.8 \mathrm{~Hz}, 2 \mathrm{H}, \mathrm{Ar}-\mathrm{H}) .{ }^{13} \mathrm{C} \mathrm{NMR}\left(75 \mathrm{MHz}, \mathrm{CDCl}_{3}\right): \delta=14.5,20.9,39.9$, 67.9, 85.4, 122.1, 129.3, 135.9, 136.2, 148.2, 154.8.; MS $\left(\mathrm{ESI}^{+}\right) \mathrm{m} / z=247(\mathrm{M}+\mathrm{H})^{+} ; \mathrm{HRMS}$ $\left(\mathrm{ESI}^{+}\right)$calcd for $\mathrm{C}_{14} \mathrm{H}_{19} \mathrm{~N}_{2} \mathrm{O}_{2}(\mathrm{M}+\mathrm{H})^{+} 247.1441$, found 247.1440.

1-(3-Chlorophenyl)-5-ethoxy-3-(methoxymethyl)-1H-pyrazole (8f)

Yellow liquid; Yield: 15\%; IR (KBr) 2925, 1594, 1504, 1439, 1246, 1096, 778, $682 \mathrm{~cm}^{-1}$; ${ }^{1} \mathrm{H}$ $\operatorname{NMR}\left(300 \mathrm{MHz}, \mathrm{CDCl}_{3}\right): \delta=1.46\left(\mathrm{t}, J=7.0,3 \mathrm{H}, \mathrm{CH}_{3}\right), 3.42\left(\mathrm{~s}, 3 \mathrm{H}, \mathrm{OCH}_{3}\right), 4.19(\mathrm{q}, J=$ 7.0, 2H, $\left.\mathrm{OCH}_{2}\right), 4.42\left(\mathrm{~s}, 2 \mathrm{H}, \mathrm{CH}_{2}\right), 5.70(\mathrm{~s}, 1 \mathrm{H}, \mathrm{CH}), 7.21(\mathrm{dq}, J=8.0,1.0 \mathrm{~Hz}, 1 \mathrm{H}, \mathrm{Ar}-\mathrm{H})$, $7.32(\mathrm{t}, J=8.2 \mathrm{~Hz}, 1 \mathrm{H}$, Ar-H), $7.66(\mathrm{dq}, J=8.2,1.0 \mathrm{~Hz}, 1 \mathrm{H}, \mathrm{Ar}-\mathrm{H}), 7.79(\mathrm{t}, J=1.1 \mathrm{~Hz}, 1 \mathrm{H}$, Ar-H). ${ }^{13} \mathrm{C}$ NMR $\left(75 \mathrm{MHz}, \mathrm{CDCl}_{3}\right): \delta=14.5,58.2,68.1,68.9,85.6,119.5,129.8,125.9$, 129.7, 134.4, 139.6, 150.0, 155.1.; MS $\left(\mathrm{ESI}^{+}\right) \mathrm{m} / z=267(\mathrm{M}+\mathrm{H})^{+}$; HRMS (ESI $\left.{ }^{+}\right)$calcd for $\mathrm{C}_{13} \mathrm{H}_{15} \mathrm{ClN}_{2} \mathrm{O}_{2} \mathrm{Na}(\mathrm{M}+\mathrm{Na})^{+}$289.0714, found 289.0716.

5-Ethoxy-3-(methoxymethyl)-1-(3-nitrophenyl)-1H-pyrazole (8g)

Yellow liquid; Yield: 10\%; IR (KBr) 2924, 1527, 1350, 1262, 1035, 732, $678 \mathrm{~cm}^{-1}$; ${ }^{1} \mathrm{H}$ NMR $\left(300 \mathrm{MHz}, \mathrm{CDCl}_{3}\right): \delta=1.44\left(\mathrm{dt}, J=1.5,7.0 \mathrm{~Hz}, 3 \mathrm{H}, \mathrm{CH}_{3}\right), 3.42\left(\mathrm{~s}, 3 \mathrm{H}, \mathrm{CH}_{3}\right), 4.14(\mathrm{dq}, J=$ $\left.1.5,7.0 \mathrm{~Hz}, 2 \mathrm{H}, \mathrm{OCH}_{2}\right), 4.42\left(\mathrm{~s}, 2 \mathrm{H}, \mathrm{CH}_{2}\right), 5.70(\mathrm{~s}, 1 \mathrm{H}, \mathrm{CH}), 7.52(\mathrm{dd}, J=1.1,8.8 \mathrm{~Hz}, 2 \mathrm{H}$, Ar-H), $\left.7.63(\mathrm{td}, J=2.9,2 \mathrm{H}, \mathrm{Ar}-\mathrm{H}) ; \mathrm{MS}_{\left(\mathrm{ESI}^{+}\right)}\right) m / z=278(\mathrm{M}+\mathrm{H})^{+}$.

5-Ethoxy-3-(ethoxymethyl)-1-p-tolyl-1H-pyrazole (8h)

Yellow liquid; Yield: 15\%; IR (KBr) 2925, 2854, 1663, 1520, 1216, 1110, 1047, 819, 747 $\mathrm{cm}^{-1} ;{ }^{1} \mathrm{H}$ NMR $\left(300 \mathrm{MHz}, \mathrm{CDCl}_{3}\right): \delta=1.24\left(\mathrm{t}, J=7.0 \mathrm{~Hz}, 3 \mathrm{H}, \mathrm{CH}_{3}\right), 1.42(\mathrm{t}, J=7.0 \mathrm{~Hz}, 3 \mathrm{H}$, $\left.\mathrm{CH}_{3}\right), 2.34\left(\mathrm{~s}, 3 \mathrm{H}, \mathrm{CH}_{3}\right), 3.59$ (q, $\left.J=7.0 \mathrm{~Hz}, 2 \mathrm{H}, \mathrm{OCH}_{2}\right), 4.15$ (q, $J=7.0 \mathrm{~Hz}, 2 \mathrm{H}, \mathrm{OCH}_{2}$ ), $4.47\left(\mathrm{~s}, 2 \mathrm{H}, \mathrm{CH}_{2}\right), 5.71(\mathrm{~s}, 1 \mathrm{H}, \mathrm{CH}), 7.20(\mathrm{~d}, J=8.0 \mathrm{~Hz}, 2 \mathrm{H}, \mathrm{Ar}-\mathrm{H}), 7.56(\mathrm{~d}, J=8.3 \mathrm{~Hz}, 2 \mathrm{H}$, 
$\mathrm{Ar}-\mathrm{H}) .{ }^{13} \mathrm{C} \mathrm{NMR}\left(75 \mathrm{MHz}, \mathrm{CDCl}_{3}\right): \delta=14.6,15.2,20.9,65.8,67.2,67.7,85.3,122.1,129.1$, 135.9, 136.1, 149.5, 154.8.; MS $\left(\mathrm{ESI}^{+}\right) \mathrm{m} / z=261(\mathrm{M}+\mathrm{H})^{+}$; HRMS $\left(\mathrm{ESI}^{+}\right)$calcd for $\mathrm{C}_{15} \mathrm{H}_{21} \mathrm{~N}_{2} \mathrm{O}_{2}(\mathrm{M}+\mathrm{H})^{+}$261.1597, found 261.1598.

1-(3-Chlorophenyl)-5-ethoxy-3-(ethoxymethyl)-1H-pyrazole (8i)

Yellow liquid; Yield: $16 \%$; ${ }^{1} \mathrm{H}$ NMR $\left(300 \mathrm{MHz}, \mathrm{CDCl}_{3}\right): \delta=1.25\left(\mathrm{t}, J=7.0 \mathrm{~Hz}, 3 \mathrm{H}, \mathrm{CH}_{3}\right)$, $1.46\left(\mathrm{t}, J=7.0 \mathrm{~Hz}, 3 \mathrm{H}, \mathrm{CH}_{3}\right), 3.60\left(\mathrm{q}, J=7.0 \mathrm{~Hz}, 2 \mathrm{H}, \mathrm{OCH}_{2}\right), 4.19(\mathrm{q}, J=7.0 \mathrm{~Hz}, 2 \mathrm{H}$, $\left.\mathrm{OCH}_{2}\right), 4.47\left(\mathrm{~s}, 2 \mathrm{H}, \mathrm{CH}_{2}\right), 5.72(\mathrm{~s}, 1 \mathrm{H}, \mathrm{CH}), 7.21$ (ddd, $J=8.0,1.9$ and $\left.0.9 \mathrm{~Hz}, 1 \mathrm{H}, \mathrm{Ar}-\mathrm{H}\right)$, $7.32(\mathrm{t}, J=8.0 \mathrm{~Hz}, 1 \mathrm{H}, \mathrm{Ar}-\mathrm{H}), 7.65(\mathrm{ddd}, J=8.2,1.9$ and $0.9 \mathrm{~Hz}, 1 \mathrm{H}, \mathrm{Ar}-\mathrm{H}), 7.78(\mathrm{t}, J=1.9$ $\mathrm{Hz}, 1 \mathrm{H}, \mathrm{Ar}-\mathrm{H}) ; \mathrm{MS}\left(\mathrm{ESI}^{+}\right) \mathrm{m} / z=281(\mathrm{M}+\mathrm{H})^{+}$; HRMS $\left(\mathrm{ESI}^{+}\right)$calcd for $\mathrm{C}_{14} \mathrm{H}_{17} \mathrm{ClN}_{2} \mathrm{O}_{2} \mathrm{Na}$ $(\mathrm{M}+\mathrm{Na})^{+}$303.0870, found 303.0872.

Ethoxy-3-(ethoxymethyl)-1-(3-nitrophenyl)-1H-pyrazole (8j)

Yellow liquid; Yield: 11\%; IR (KBr) 2985, 2936, 1531, 1350, 1257, 1028, 736, $684 \mathrm{~cm}^{-1}$; ${ }^{1} \mathrm{H}$ NMR (300 MHz, $\left.\mathrm{CDCl}_{3}\right): \delta=1.27\left(\mathrm{t}, J=6.7 \mathrm{~Hz}, 3 \mathrm{H}, \mathrm{CH}_{3}\right), 1.5\left(\mathrm{t}, J=6.9 \mathrm{~Hz}, 3 \mathrm{H}, \mathrm{CH}_{3}\right)$, $3.61\left(\mathrm{q}, J=6.7,2 \mathrm{H}, \mathrm{OCH}_{2}\right), 4.24\left(\mathrm{q}, J=6.7 \mathrm{~Hz}, 2 \mathrm{H}, \mathrm{OCH}_{2}\right), 4.49\left(\mathrm{~s}, 2 \mathrm{H}, \mathrm{CH}_{2}\right), 5.77(\mathrm{~s}, 1 \mathrm{H}$, $\mathrm{CH}), 7.58(\mathrm{t}, J=8.3 \mathrm{~Hz}, 1 \mathrm{H}, \mathrm{Ar}-\mathrm{H}), 8.06(\mathrm{~m}, 2 \mathrm{H}, \mathrm{Ar}-\mathrm{H}), 8.71(\mathrm{~s}, 1 \mathrm{H}, \mathrm{Ar}-\mathrm{H}) ; \mathrm{MS}\left(\mathrm{ESI}^{+}\right) \mathrm{m} / \mathrm{z}$ $=292(\mathrm{M}+\mathrm{H})^{+}$. 


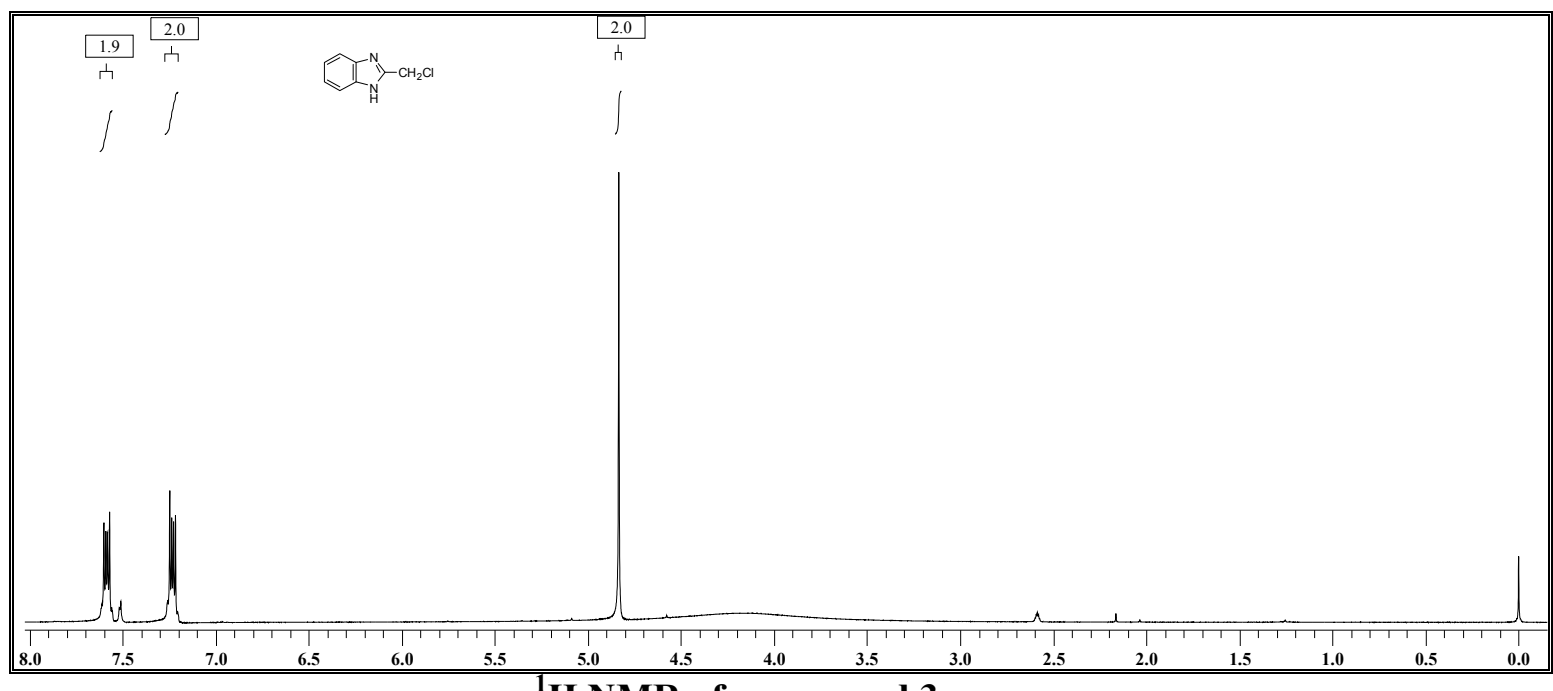

${ }^{1} H$ NMR of compound 3a

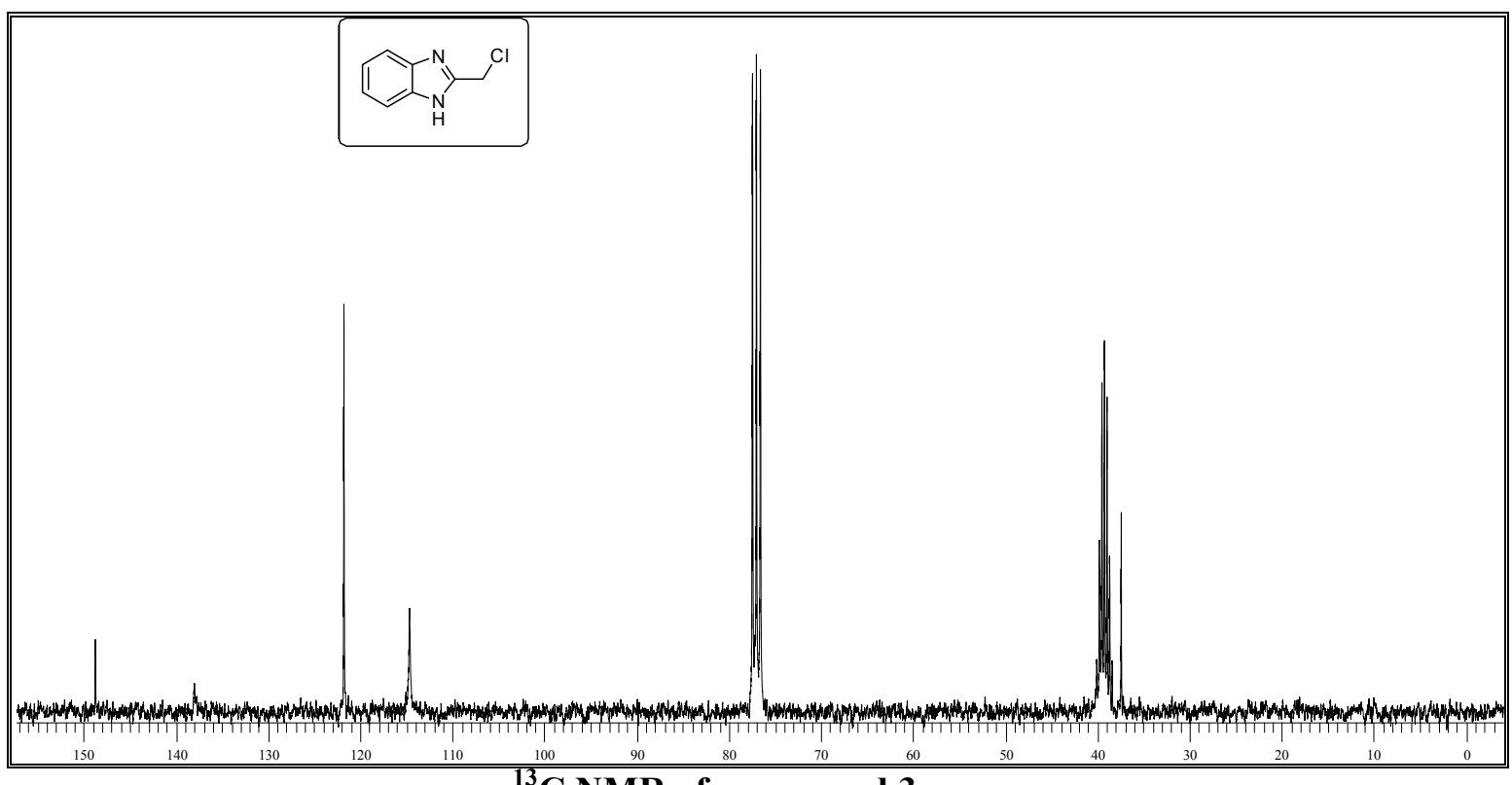

${ }^{13}$ C NMR of compound 3a 


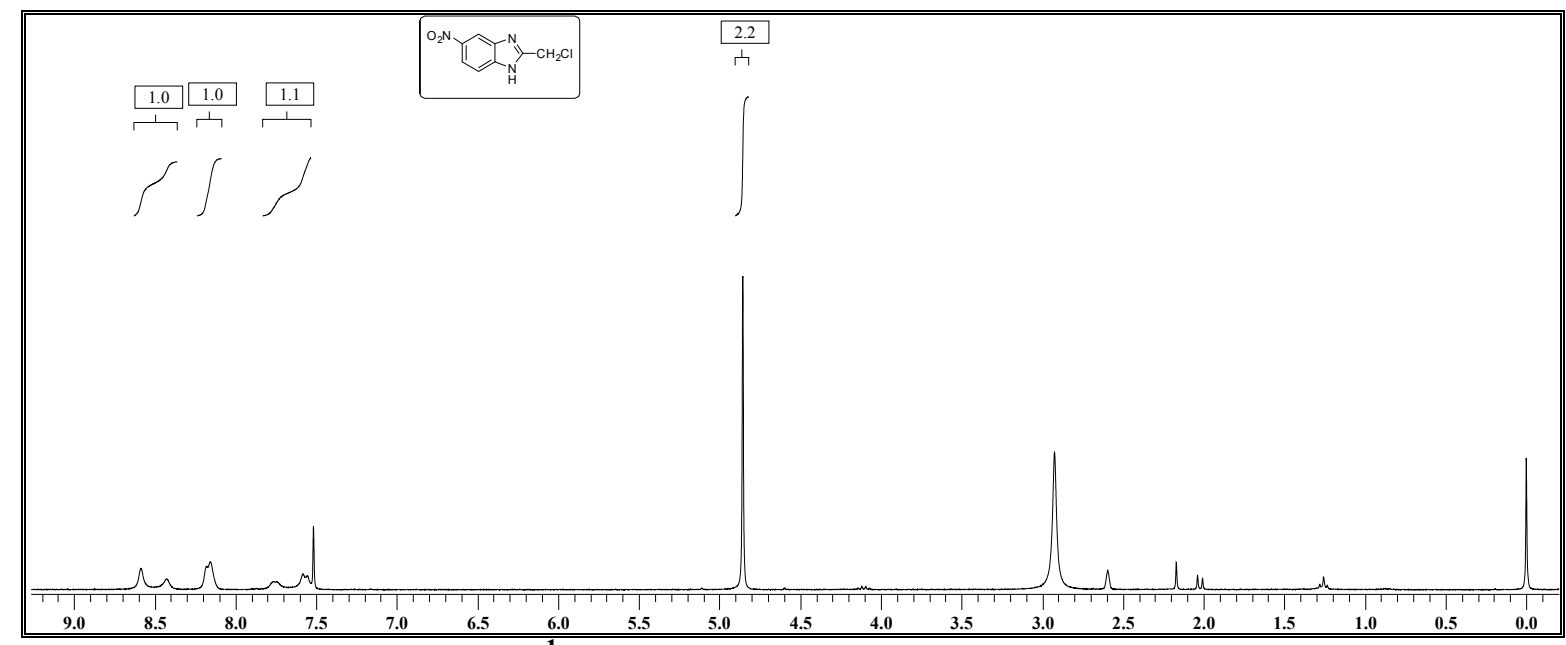

${ }^{1} \mathrm{H}$ NMR of compound $3 \mathrm{~b}$

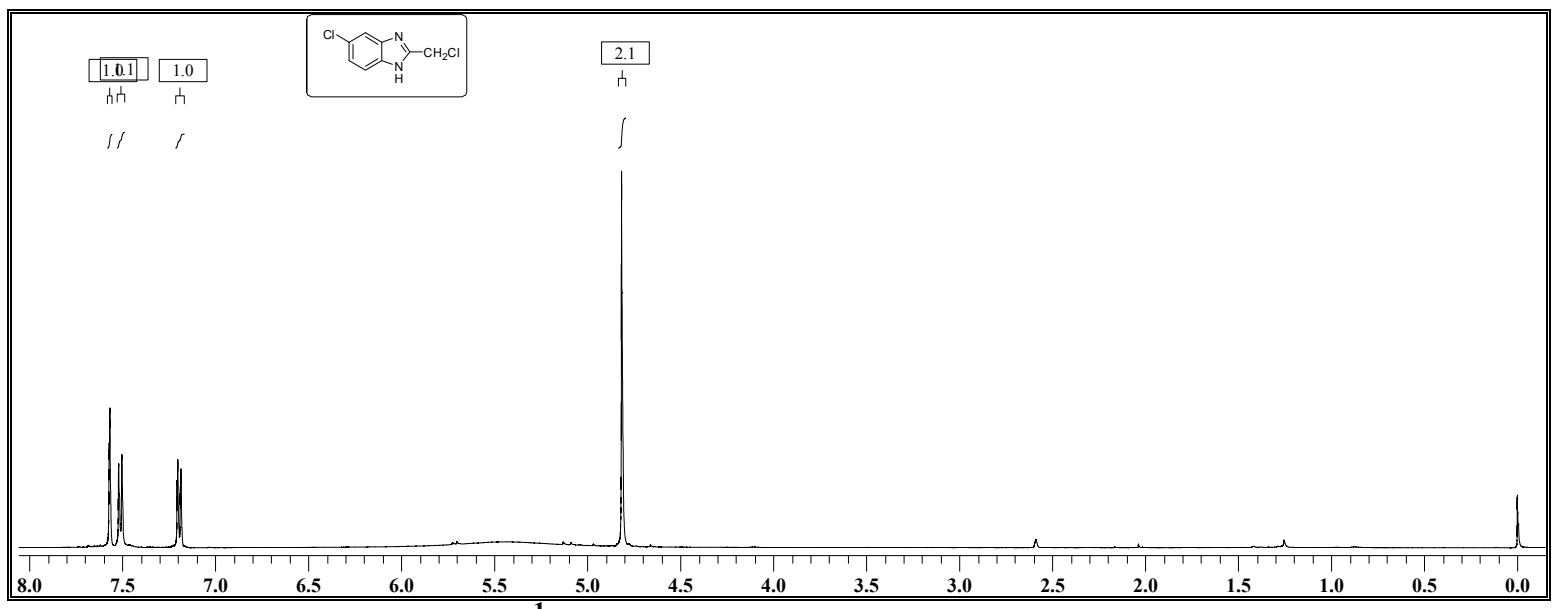

${ }^{1}$ H NMR of compound 3c

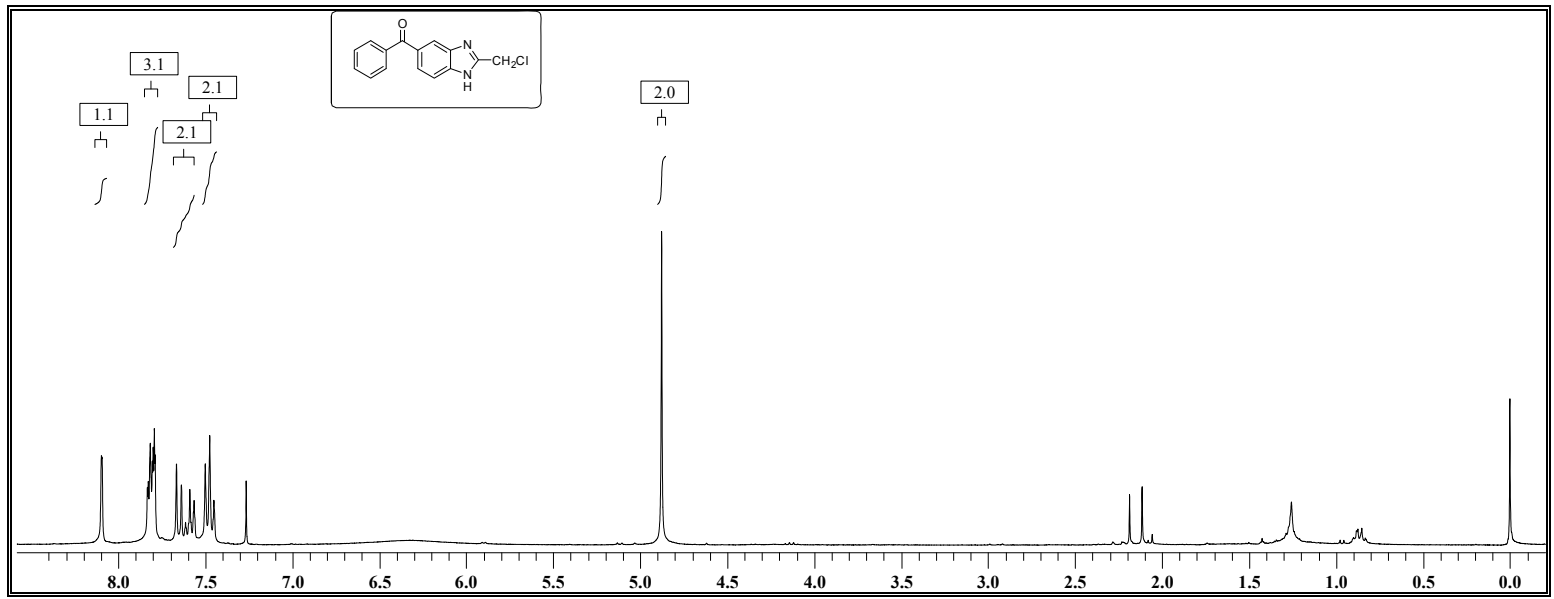


${ }^{1}$ H NMR of compound 3d

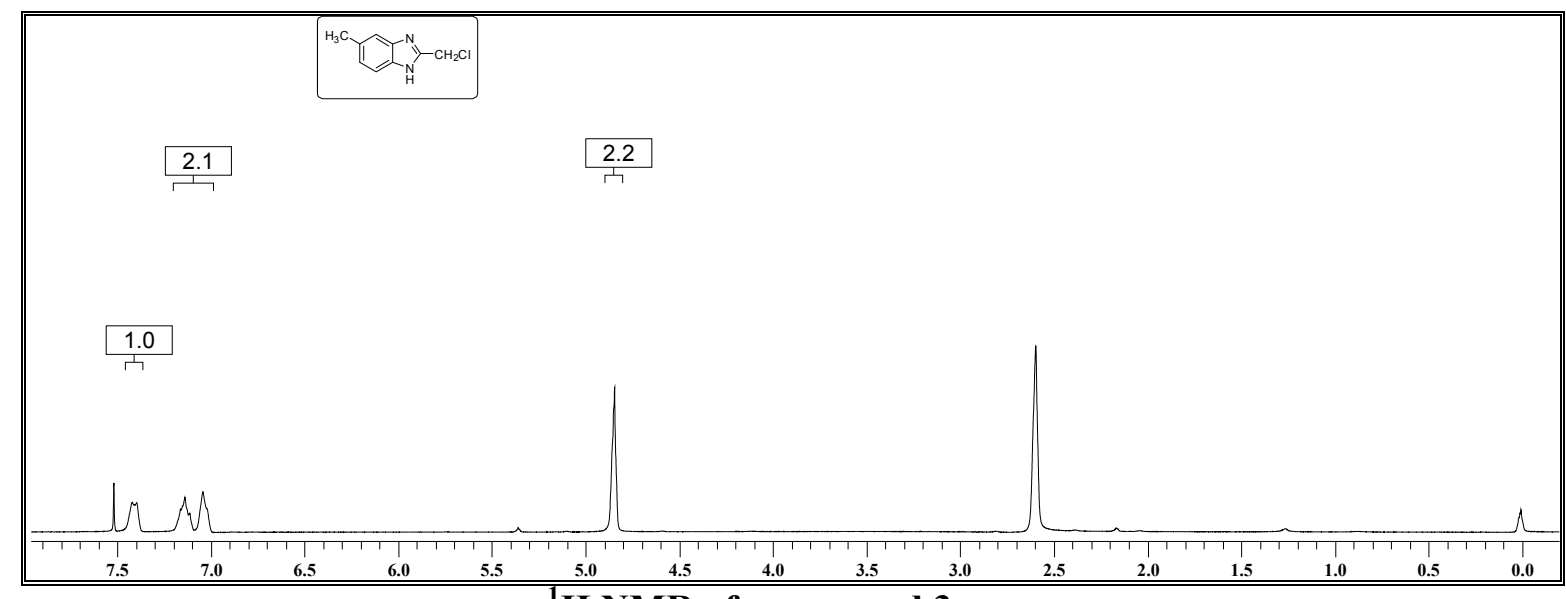

${ }^{1} \mathrm{H}$ NMR of compound $3 \mathrm{e}$

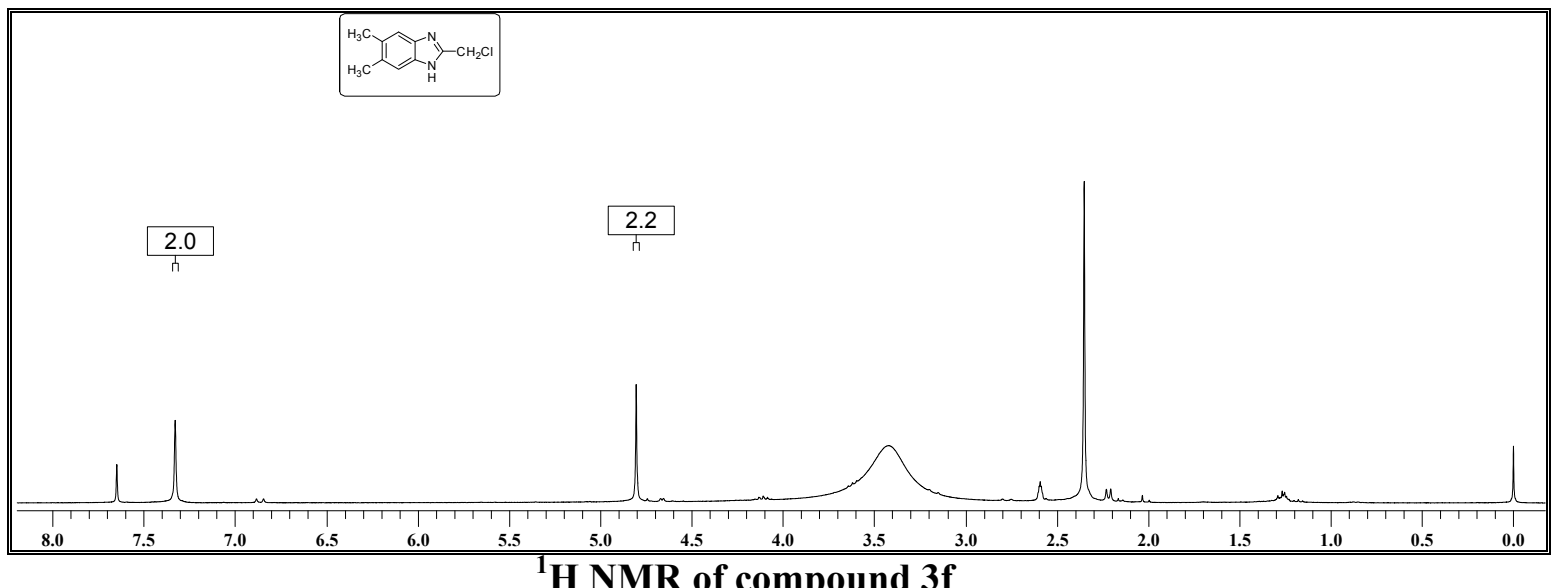

${ }^{1} H$ NMR of compound $3 f$ 

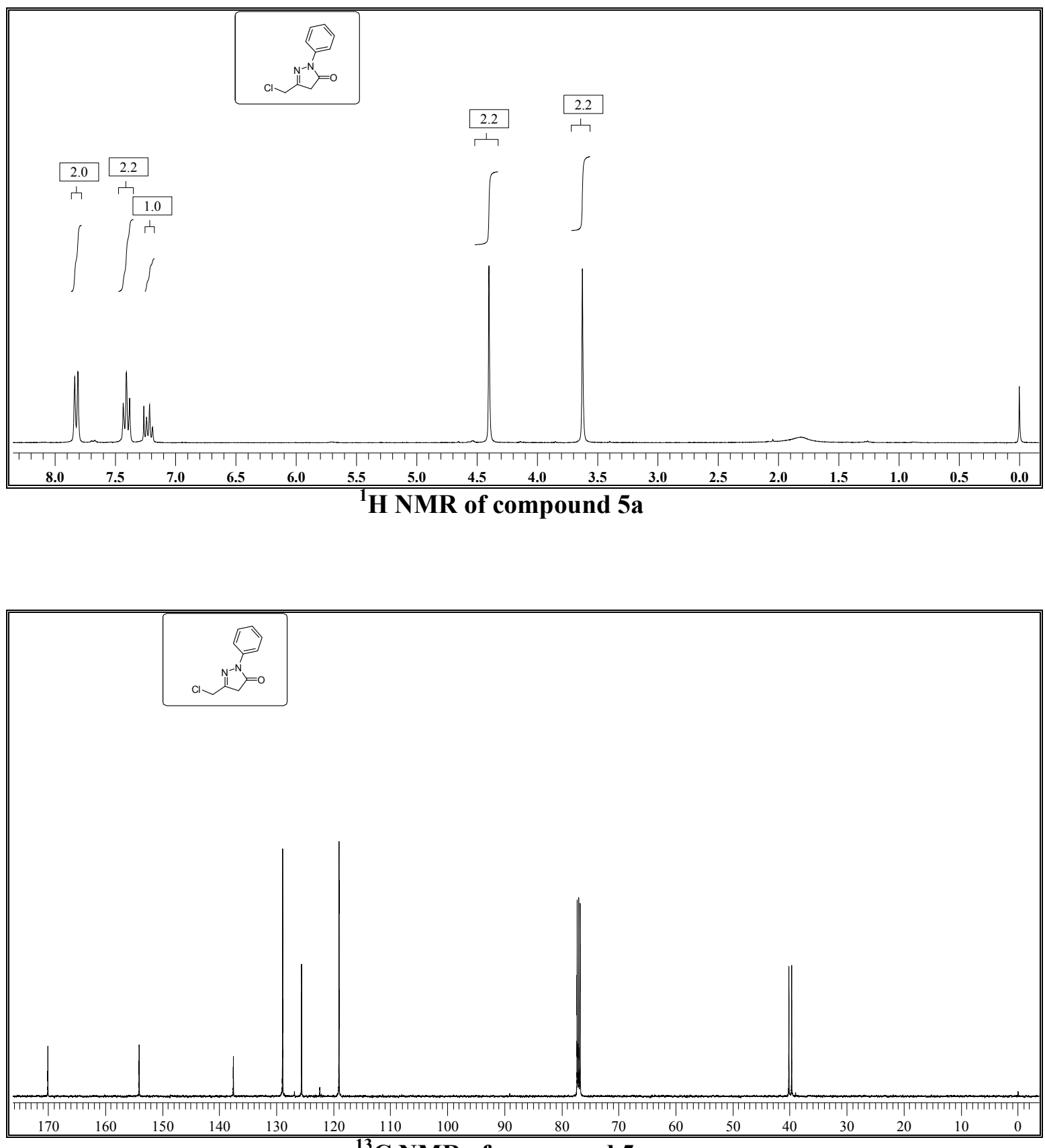

${ }^{13} \mathrm{C}$ NMR of compound $5 \mathrm{a}$ 


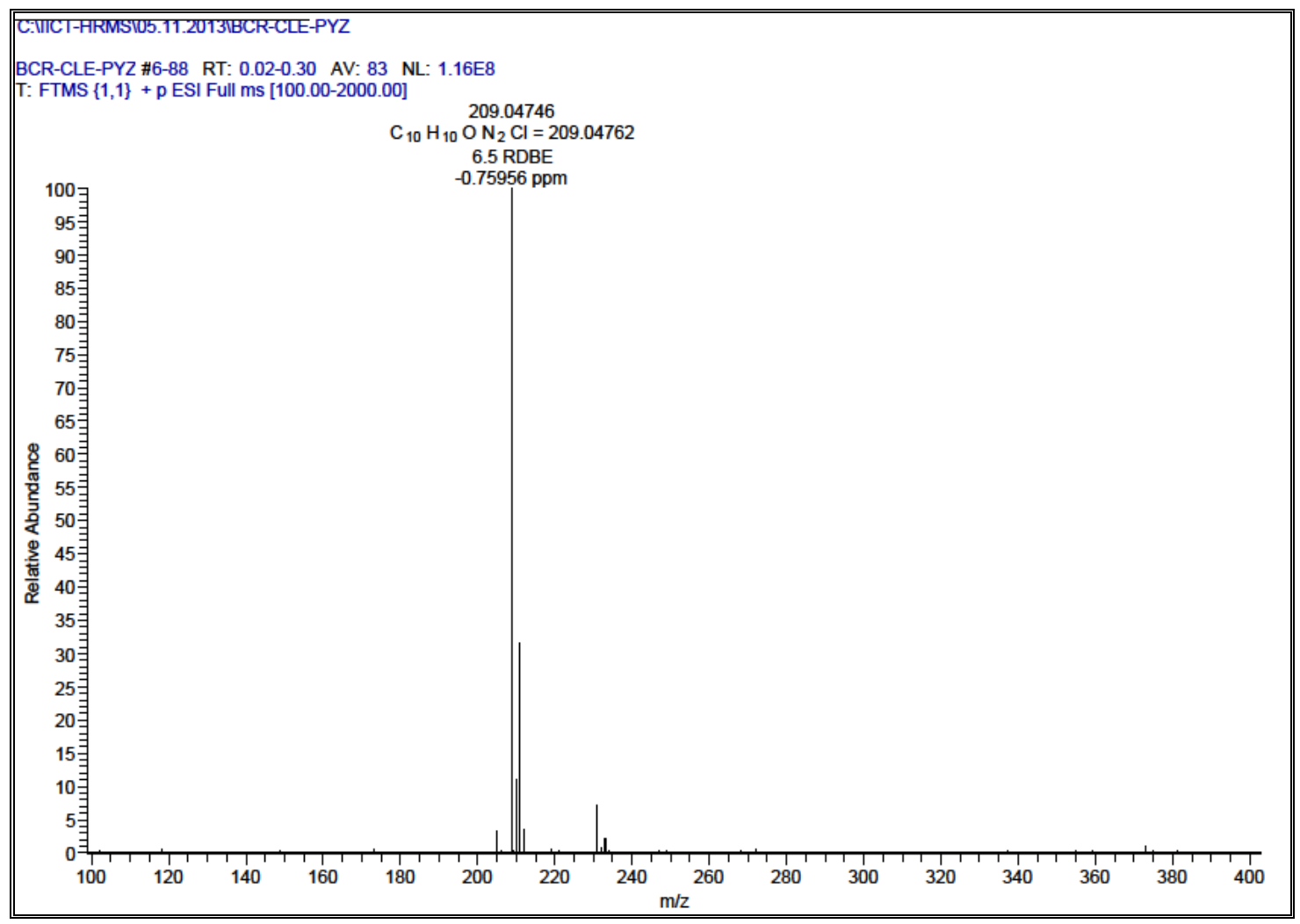

HRMS of compound 5a

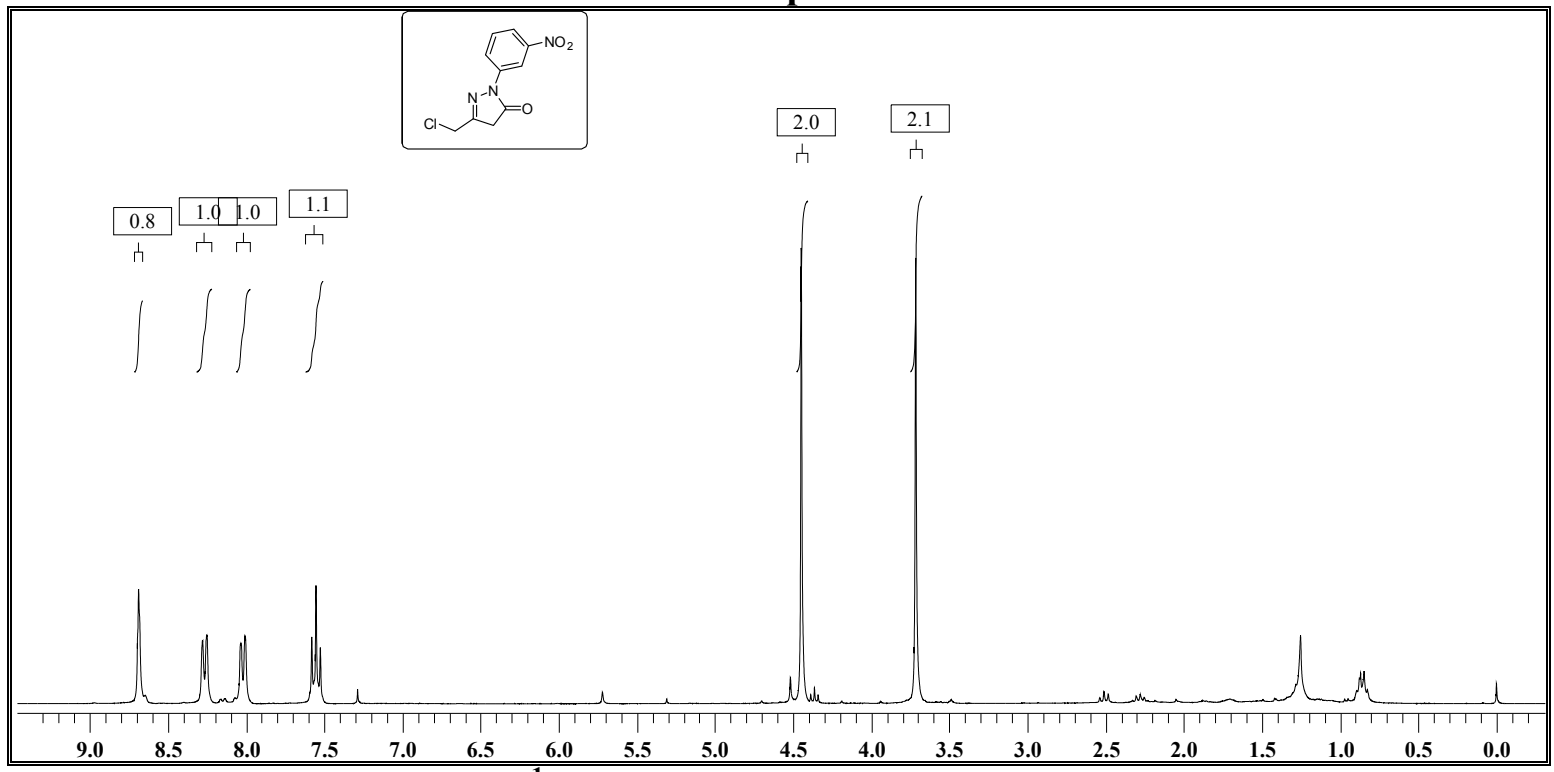

${ }^{1} \mathrm{H}$ NMR of compound $5 \mathrm{~b}$ 

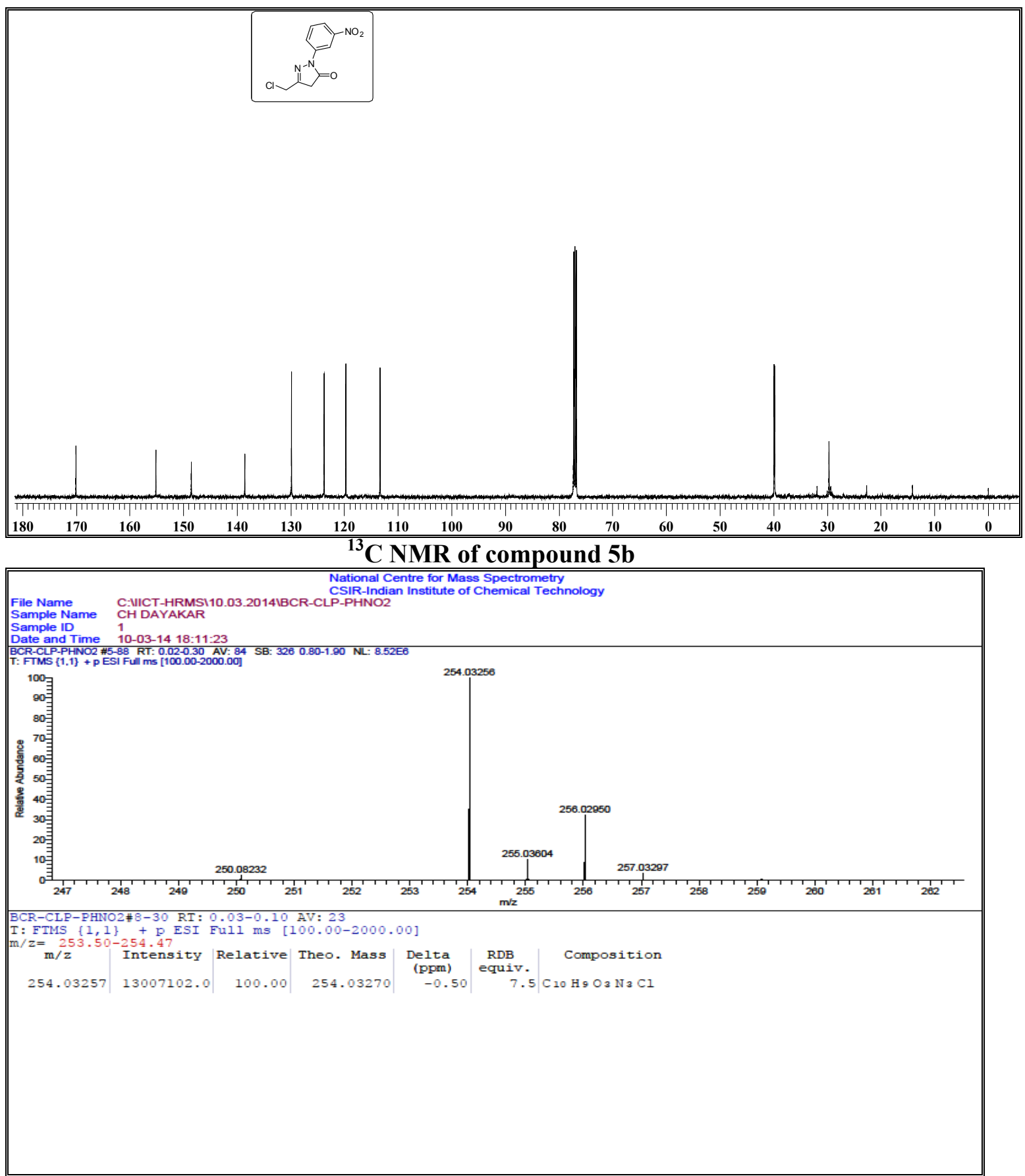

HRMS of compound $5 b$ 

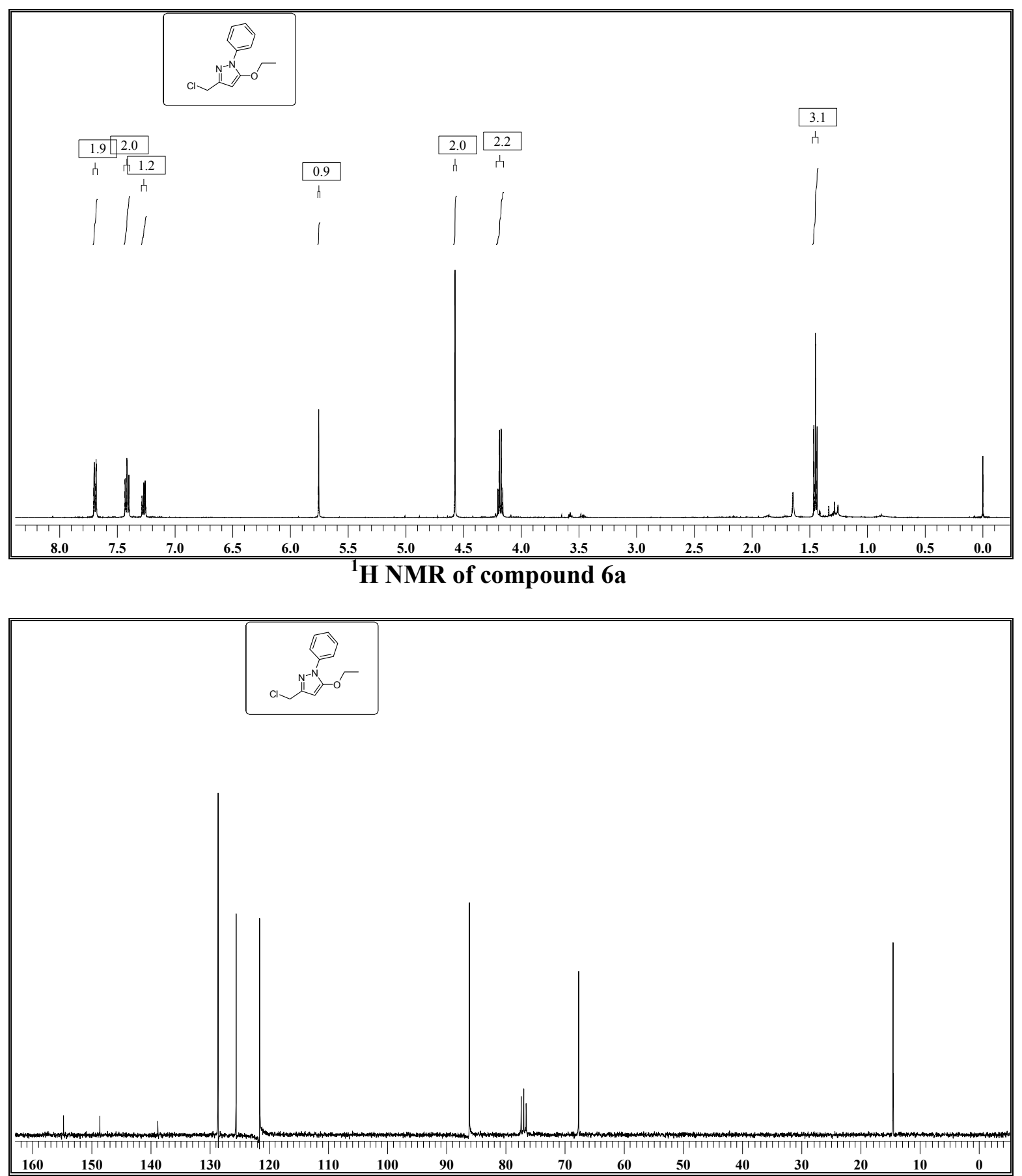

${ }^{13} \mathrm{C}$ NMR of compound $6 \mathrm{a}$ 


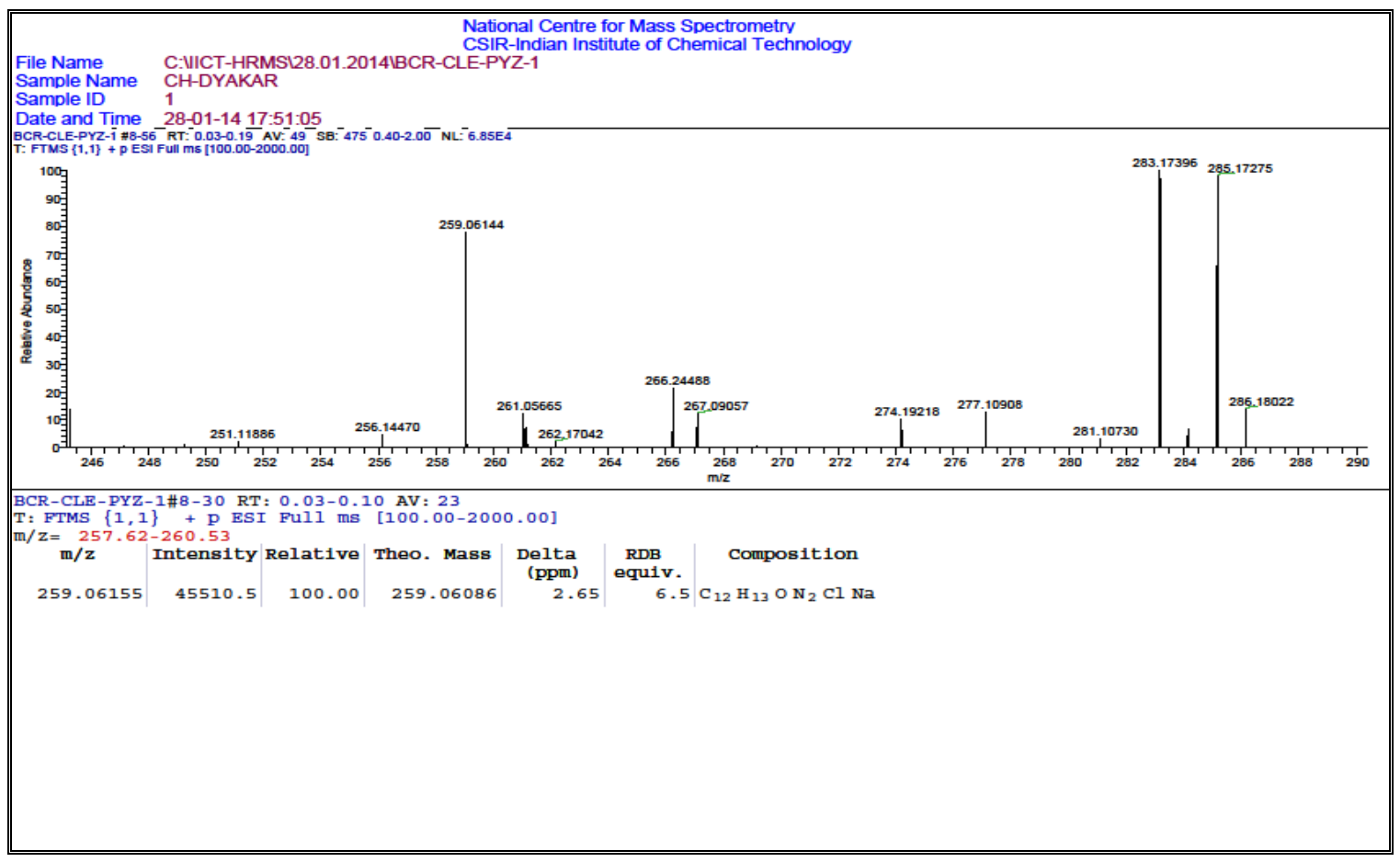

HRMS of compound 6a

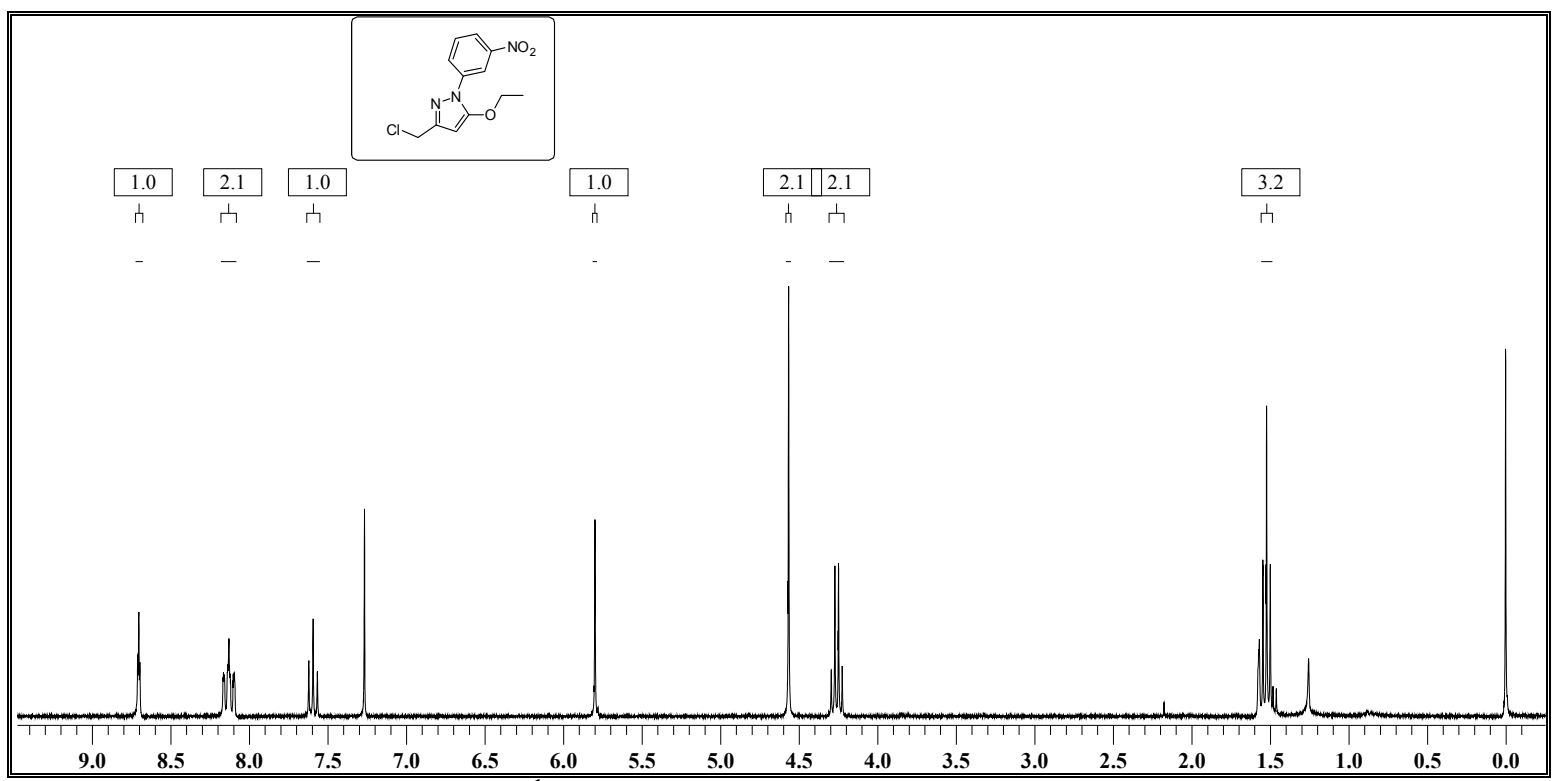

${ }^{1} H$ NMR of compound $6 \mathrm{~b}$ 


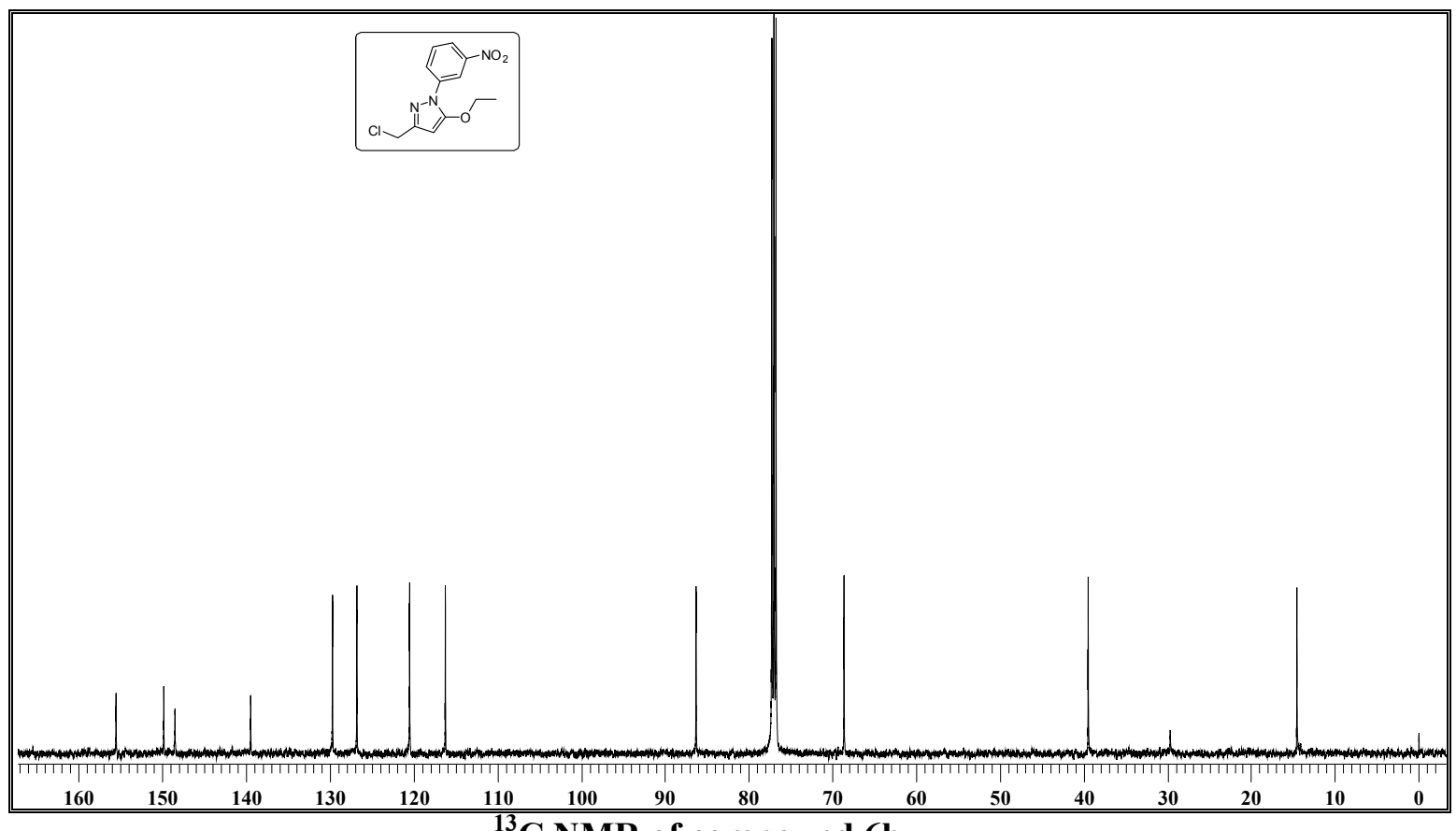

${ }^{13} \mathrm{C}$ NMR of compound $6 \mathrm{~b}$

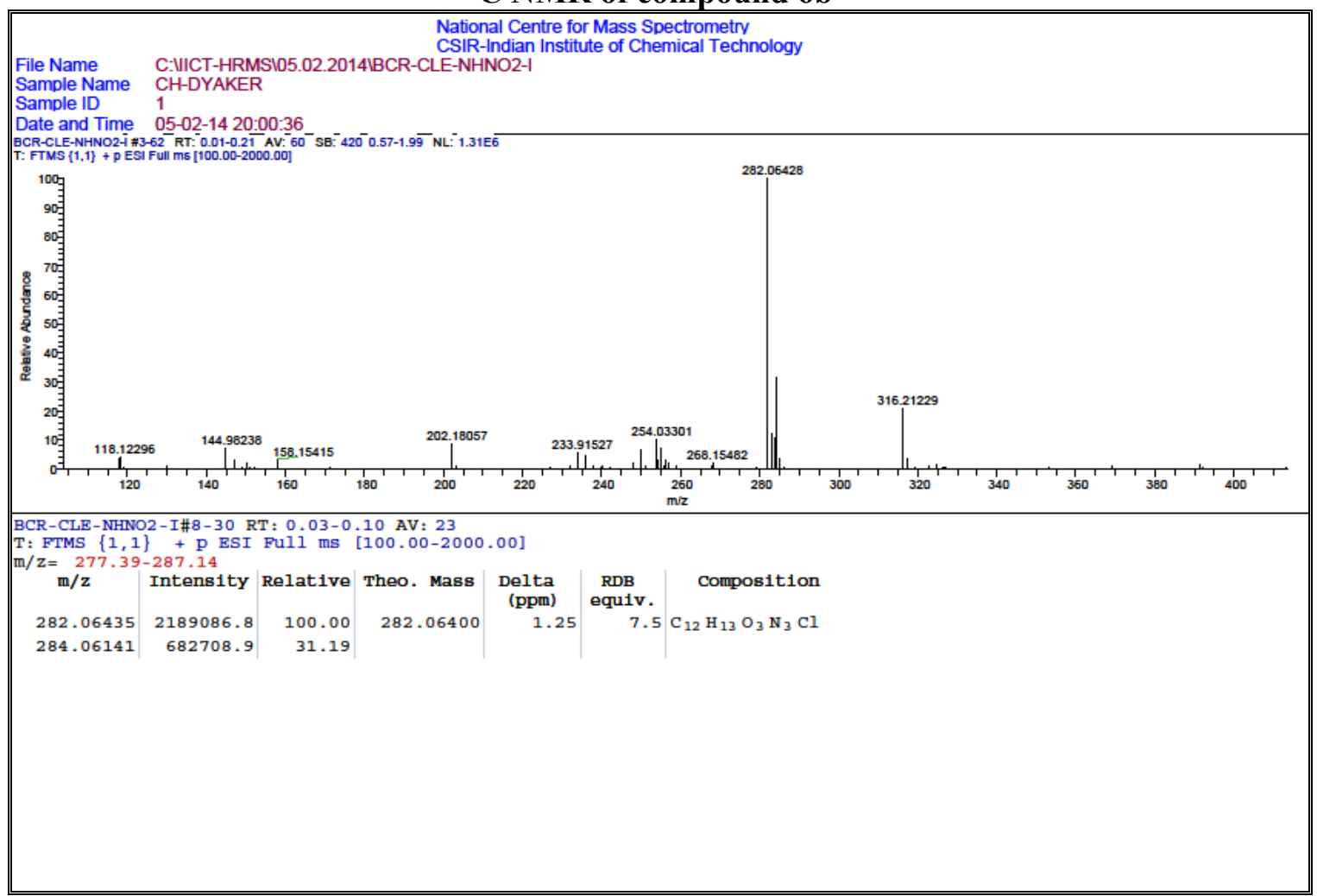

HRMS of compound $6 \mathrm{~b}$ 


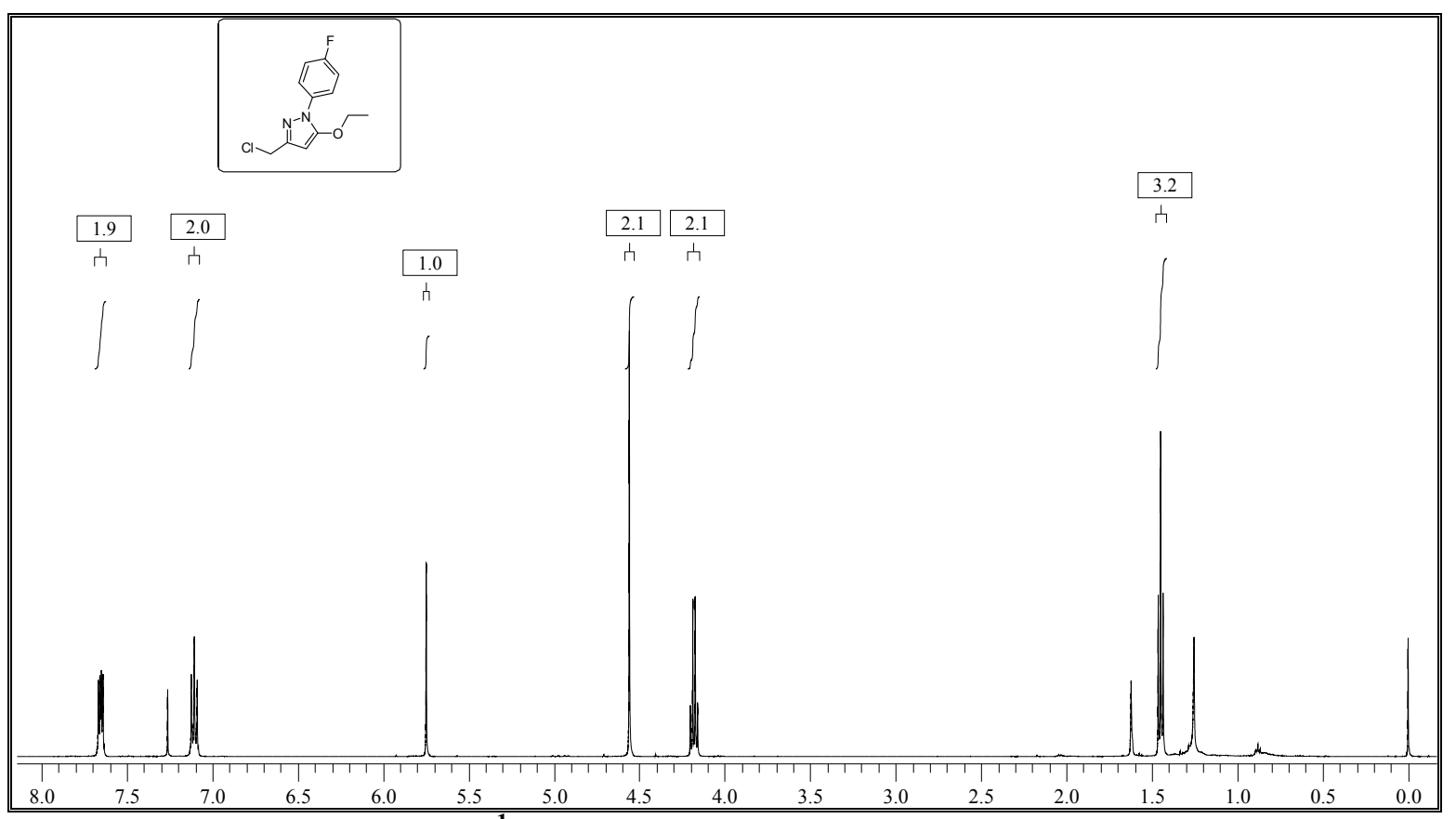

${ }^{1} \mathrm{H}$ NMR of compound $6 \mathrm{c}$

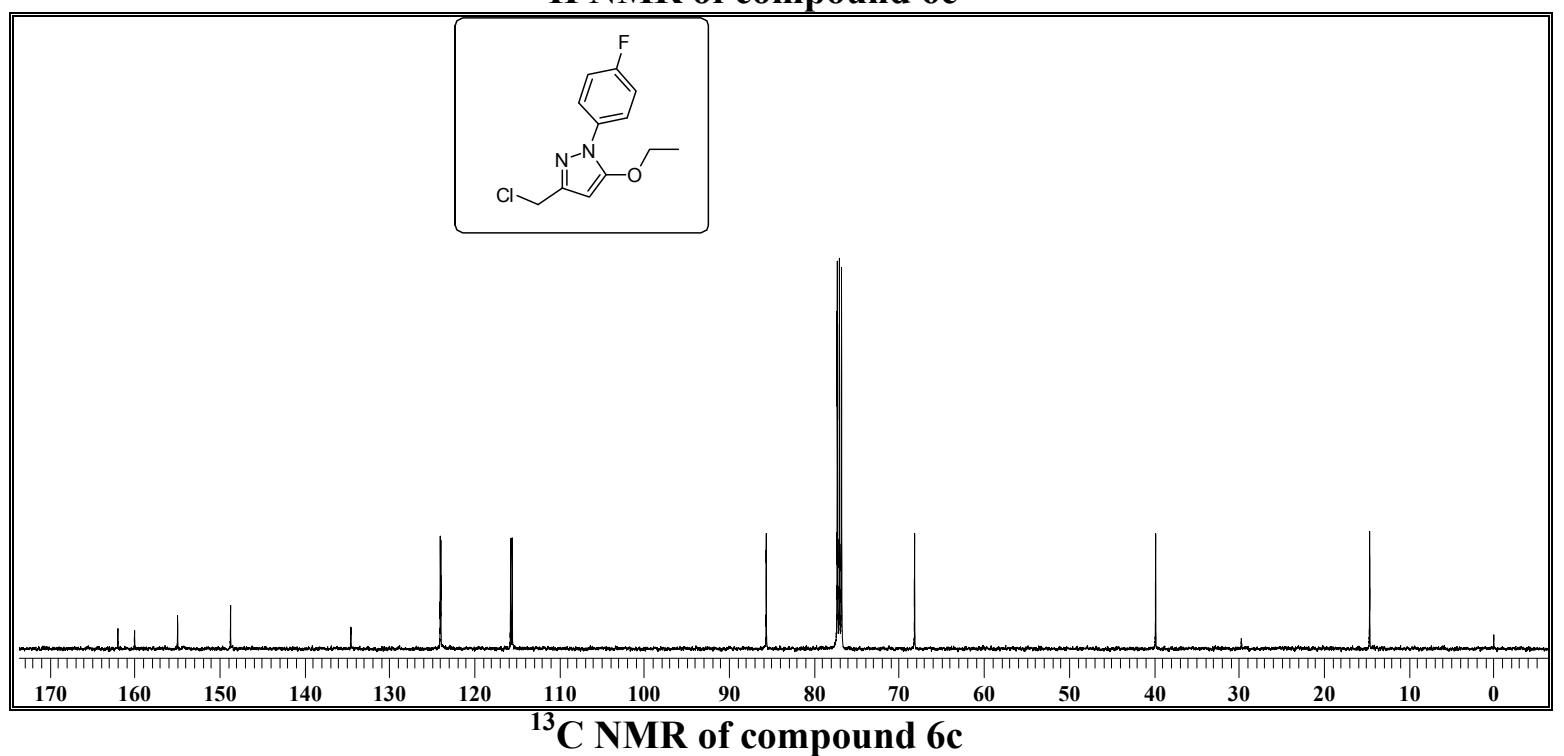




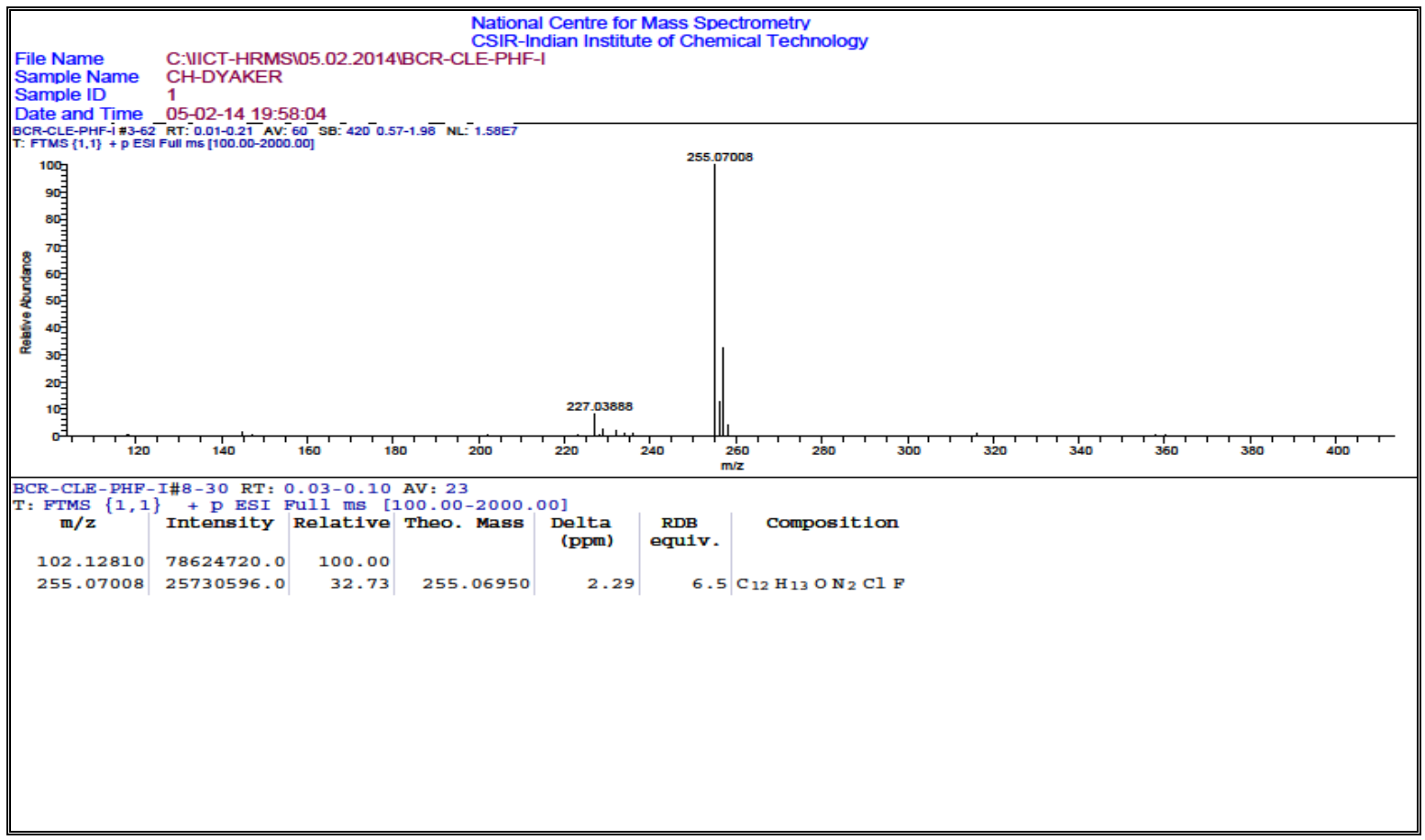

HRMS of compound 6c

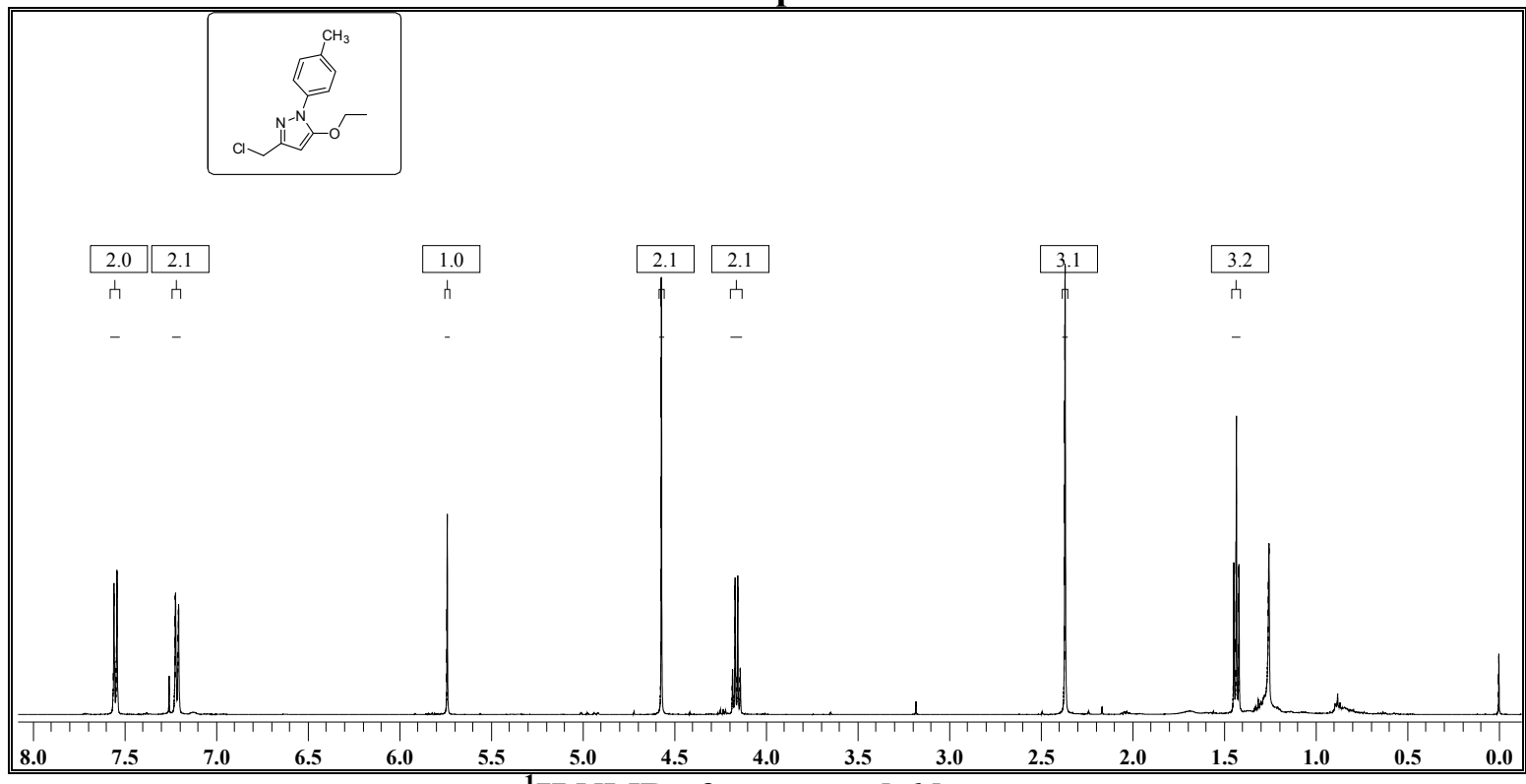

${ }^{1} H$ NMR of compound $6 d$ 


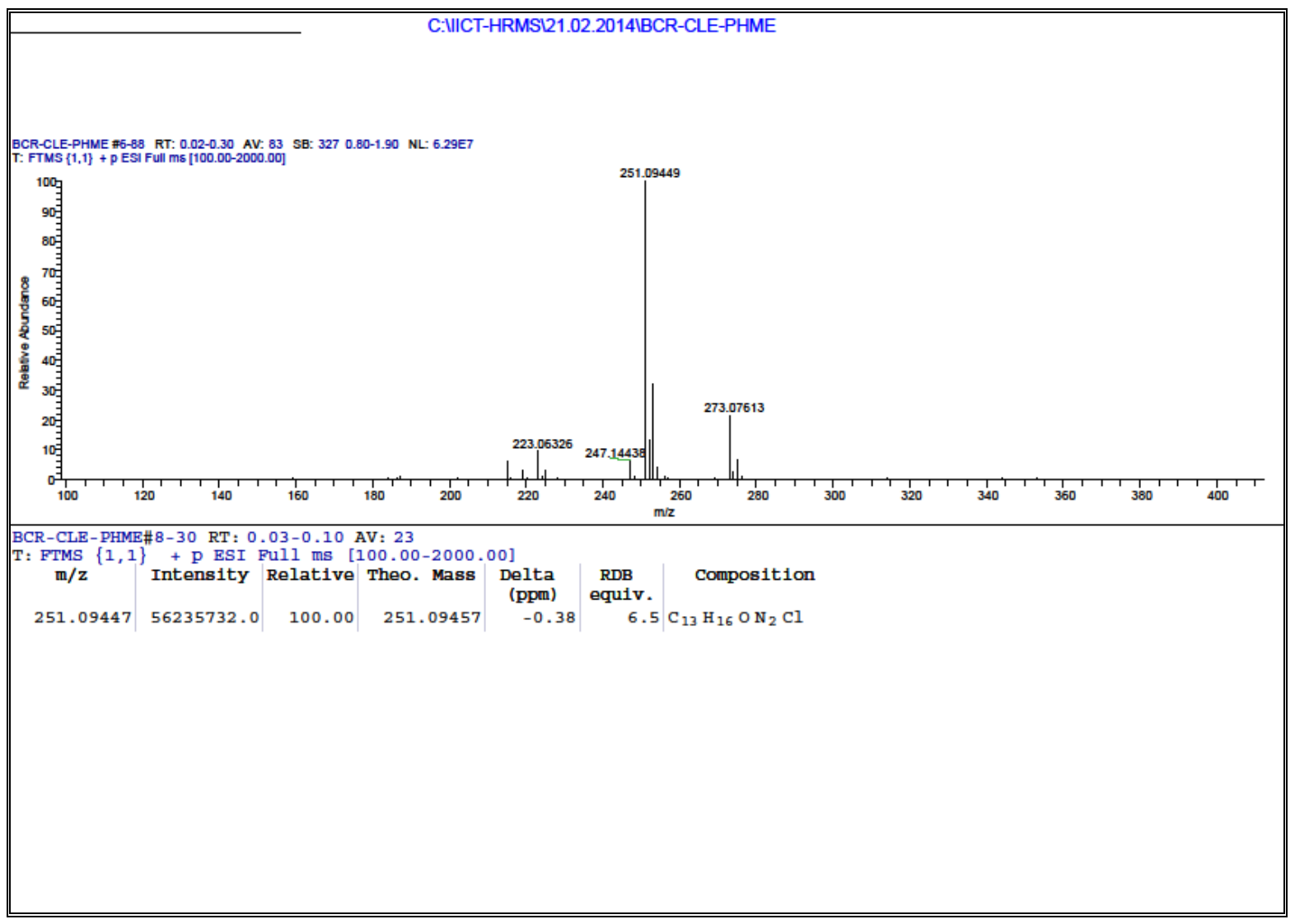

HRMS of compound 6d

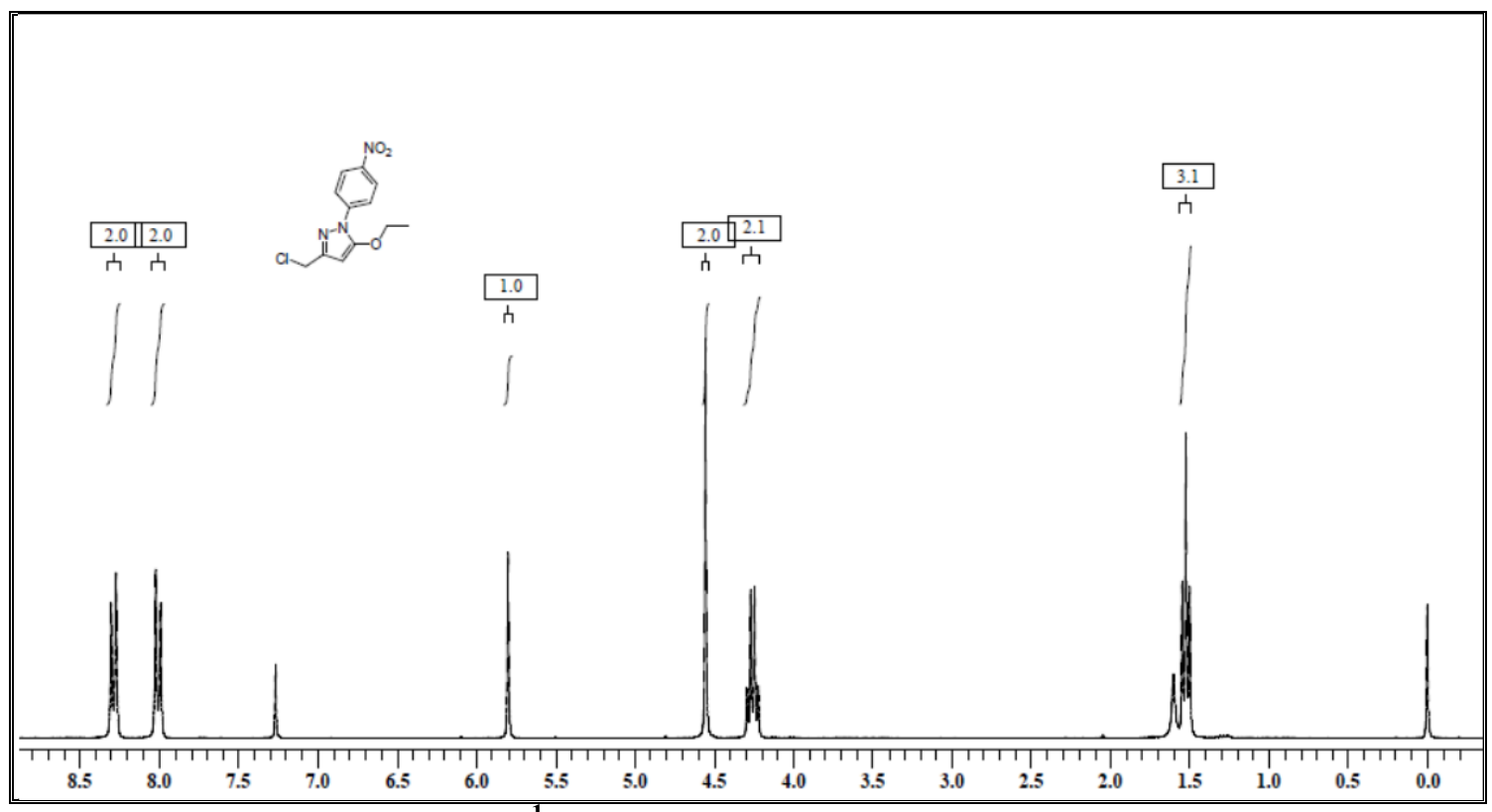

${ }^{1} \mathrm{H}$ NMR of compound $6 \mathrm{e}$ 

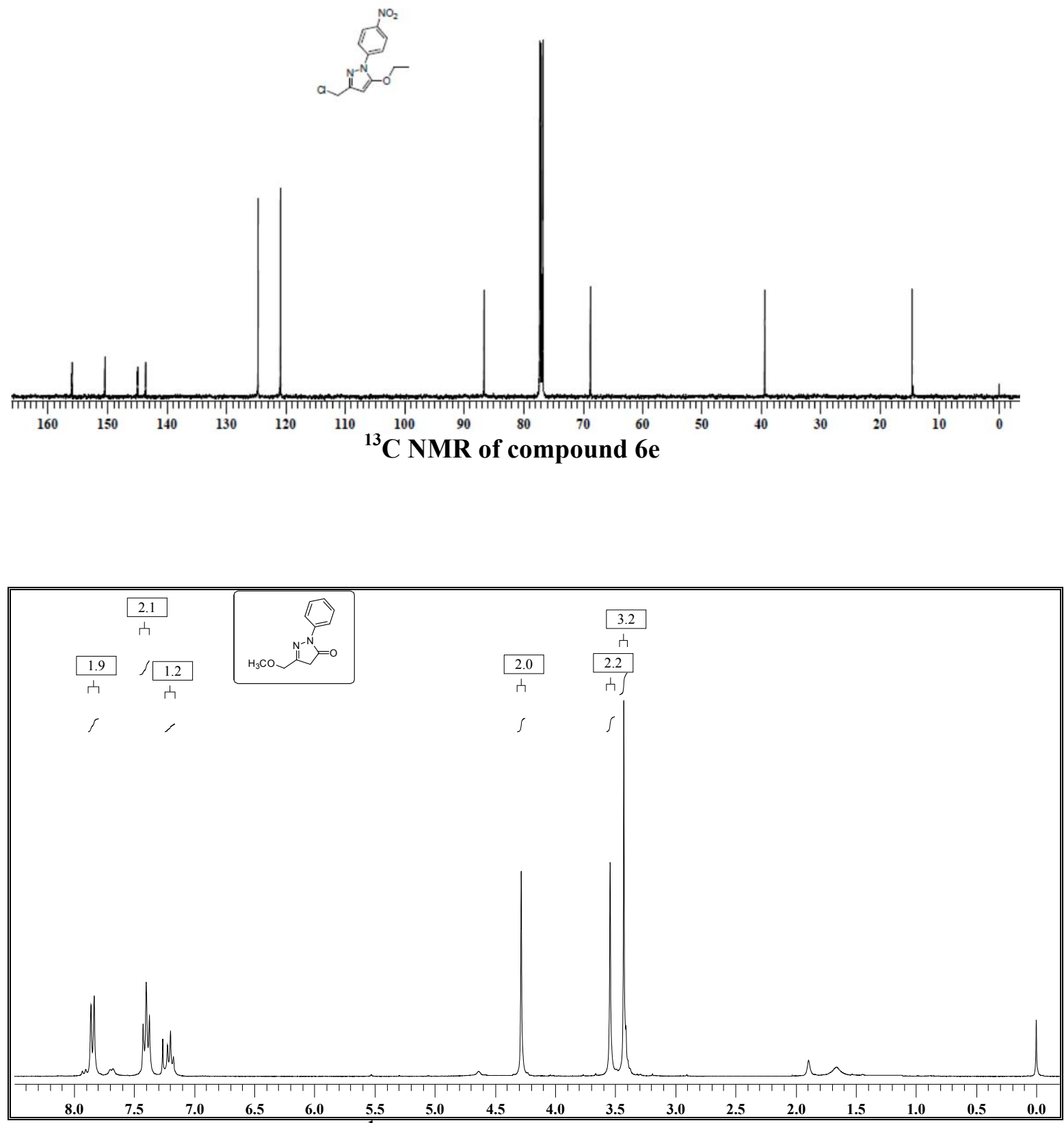

${ }^{1}$ H NMR of compound $7 \mathbf{a}$ 

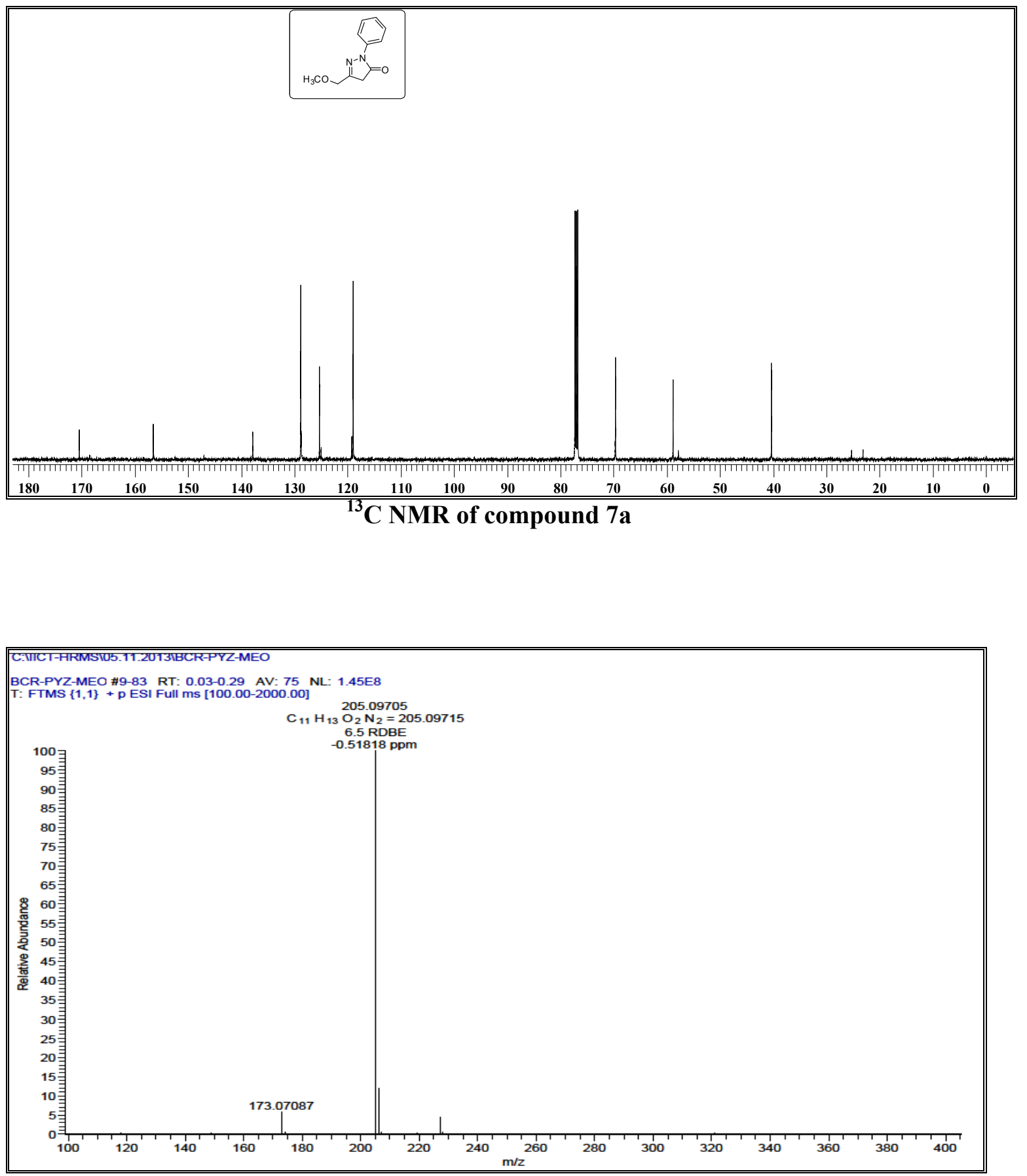

HRMS of compound 7a 


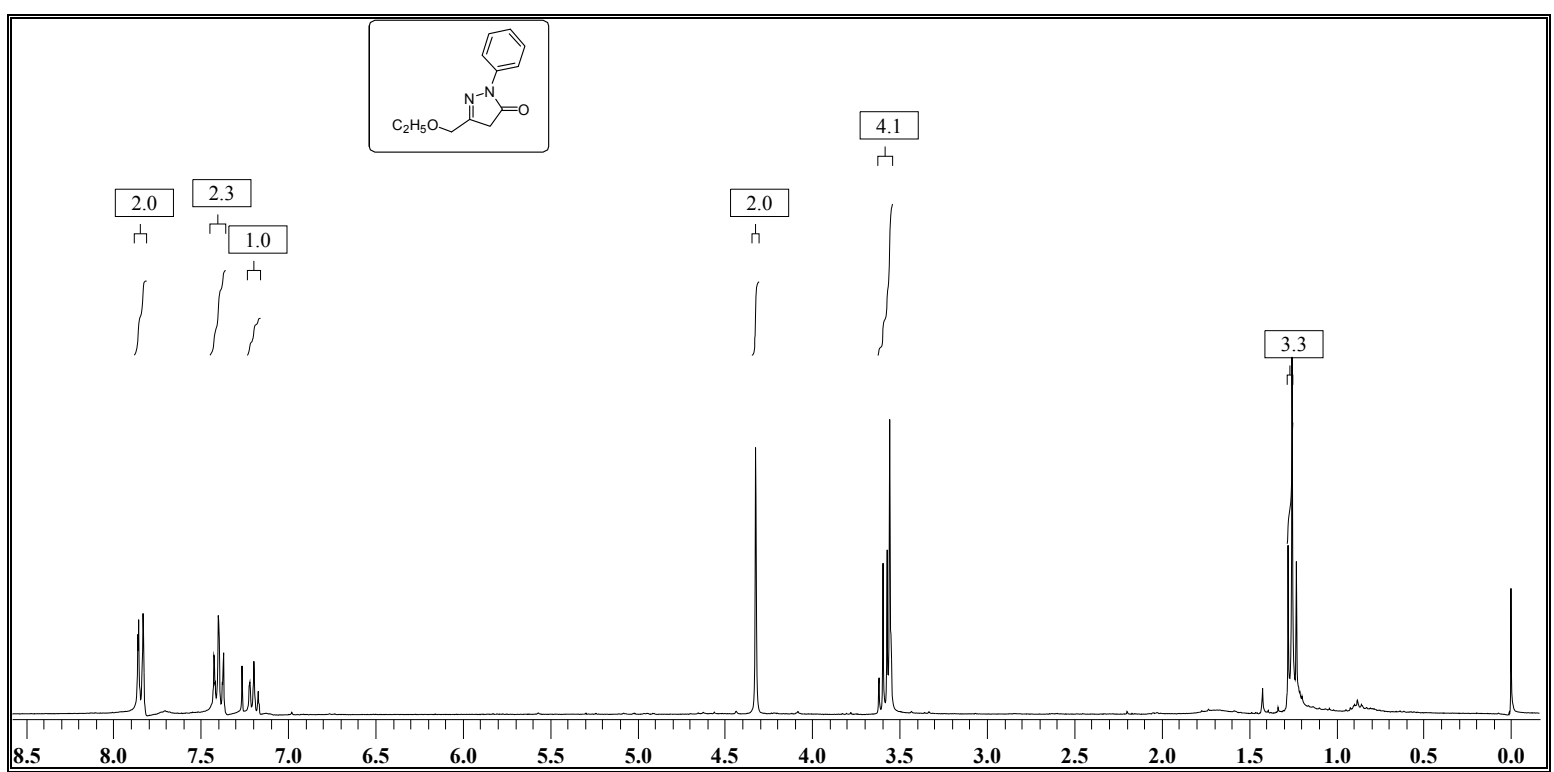

${ }^{1} \mathrm{H}$ NMR of compound $7 \mathrm{~b}$

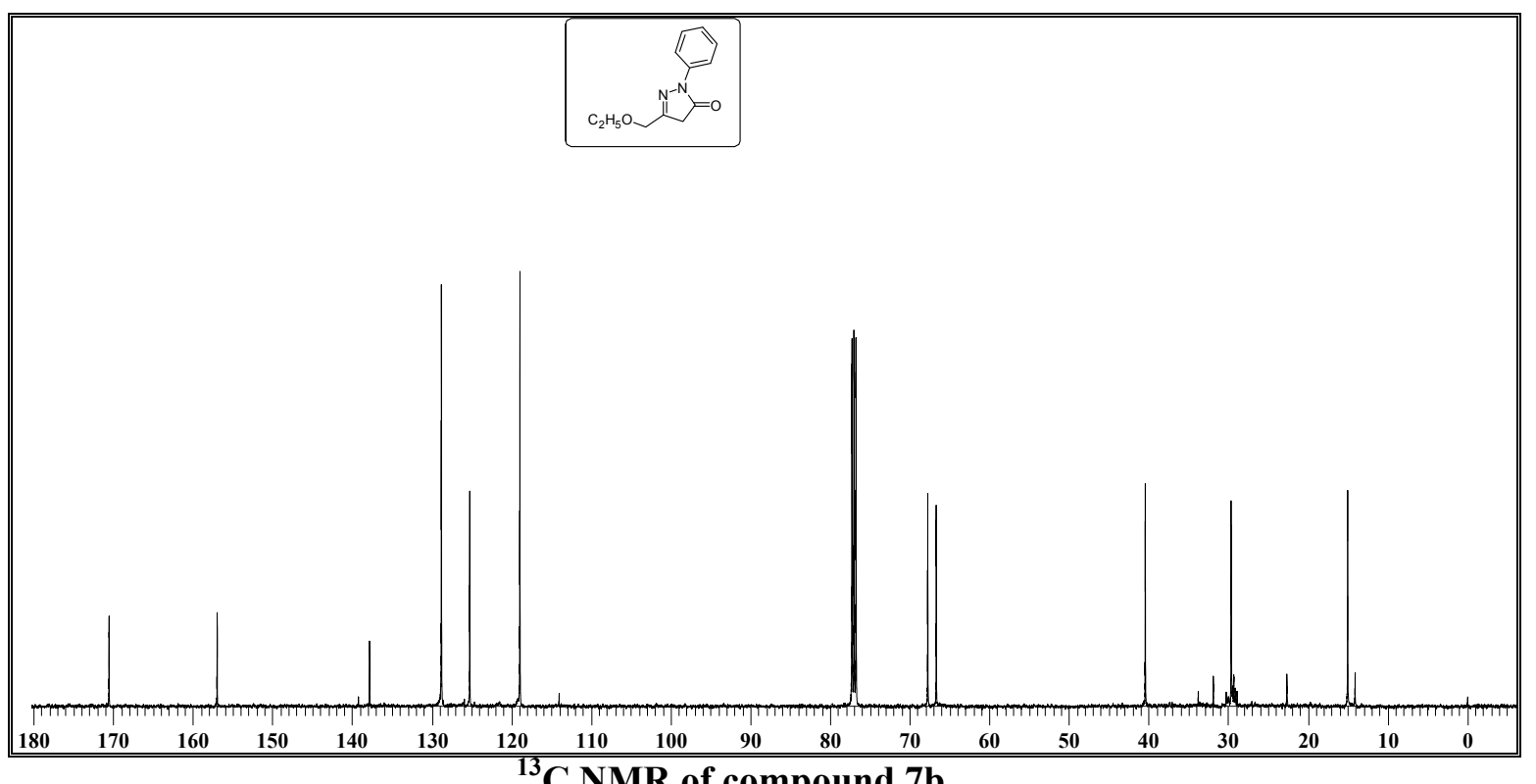

${ }^{13} \mathrm{C}$ NMR of compound $7 \mathrm{~b}$ 


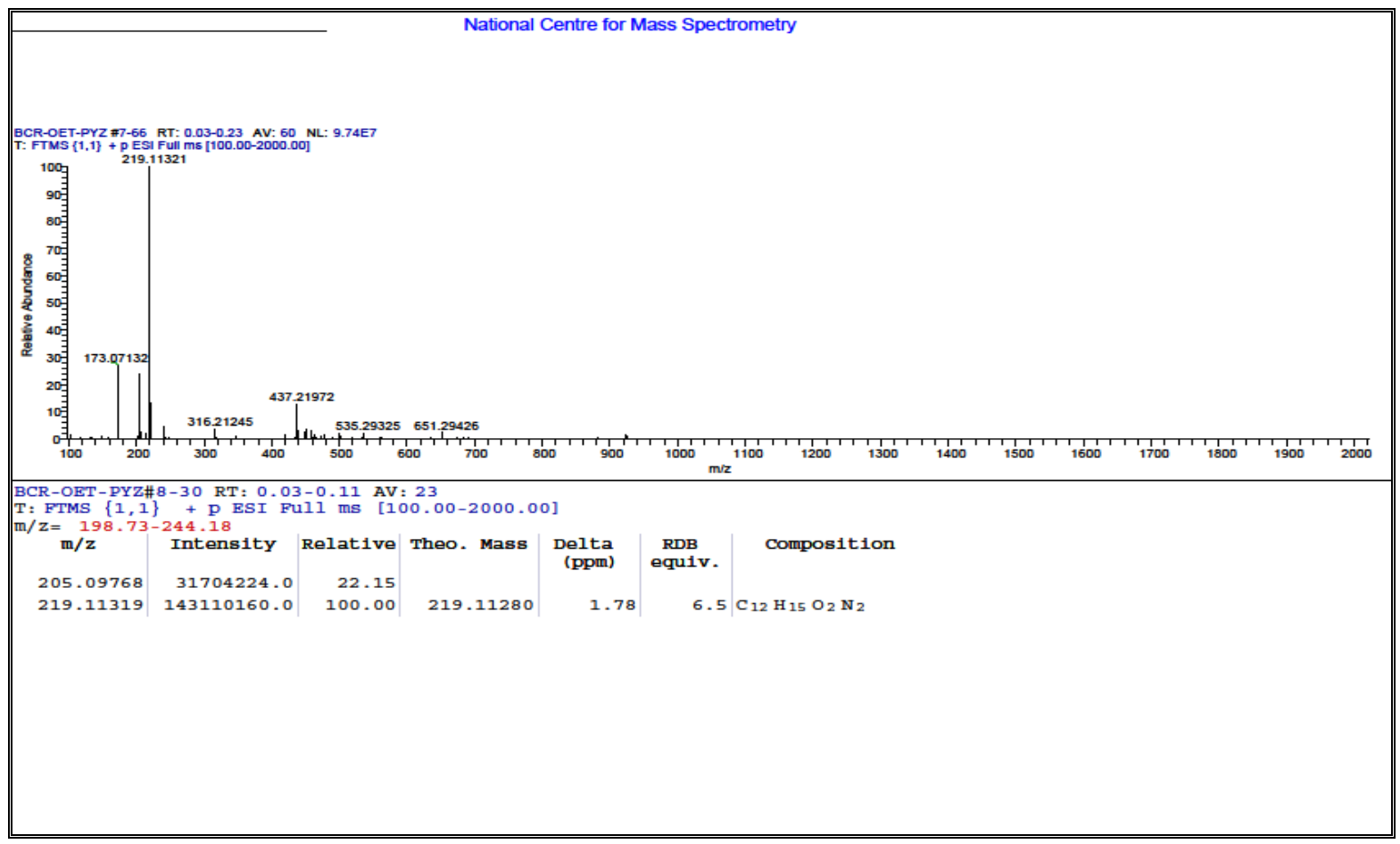

HRMS of compound $7 b$

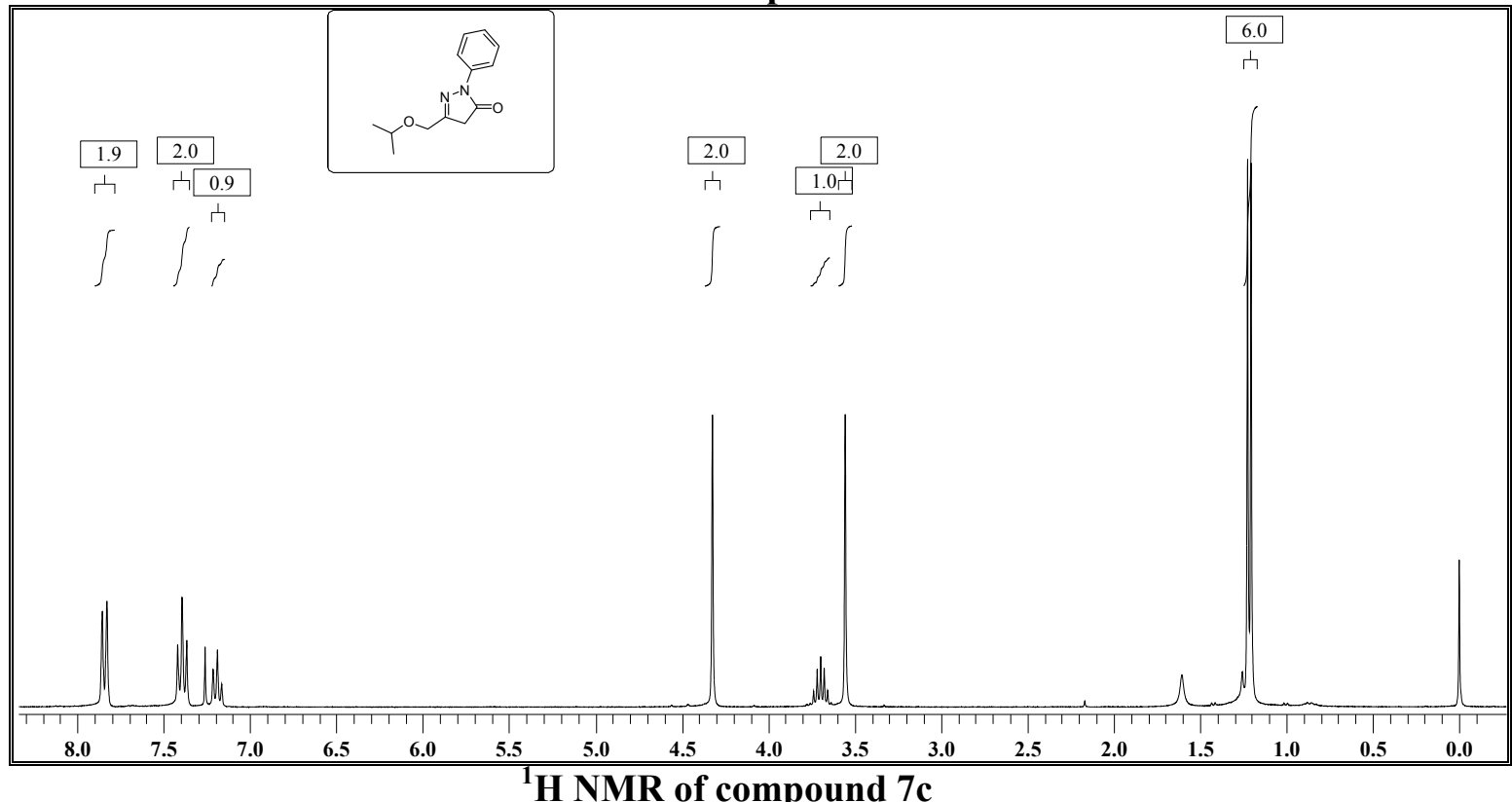



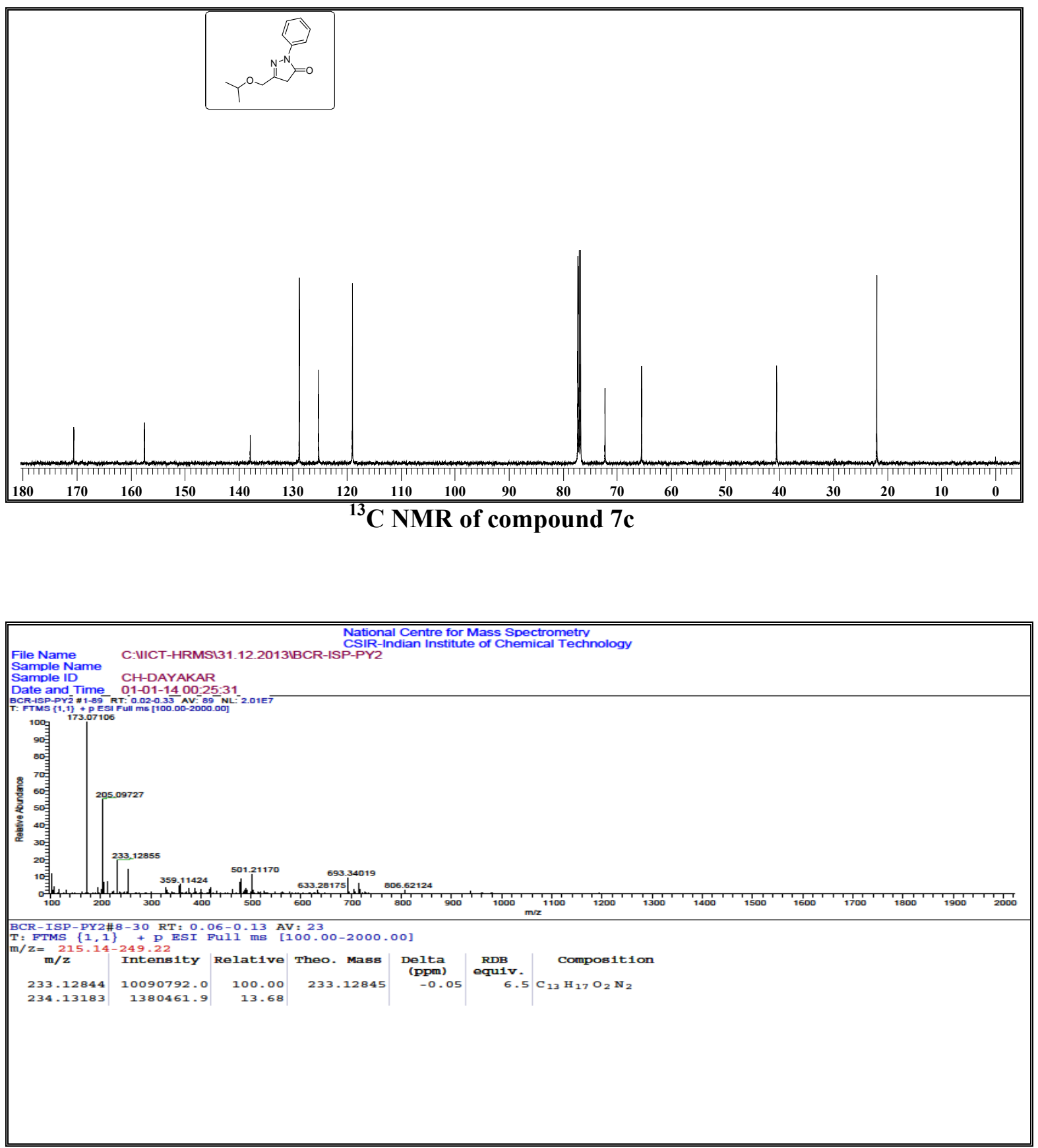

HRMS of compound 7c 


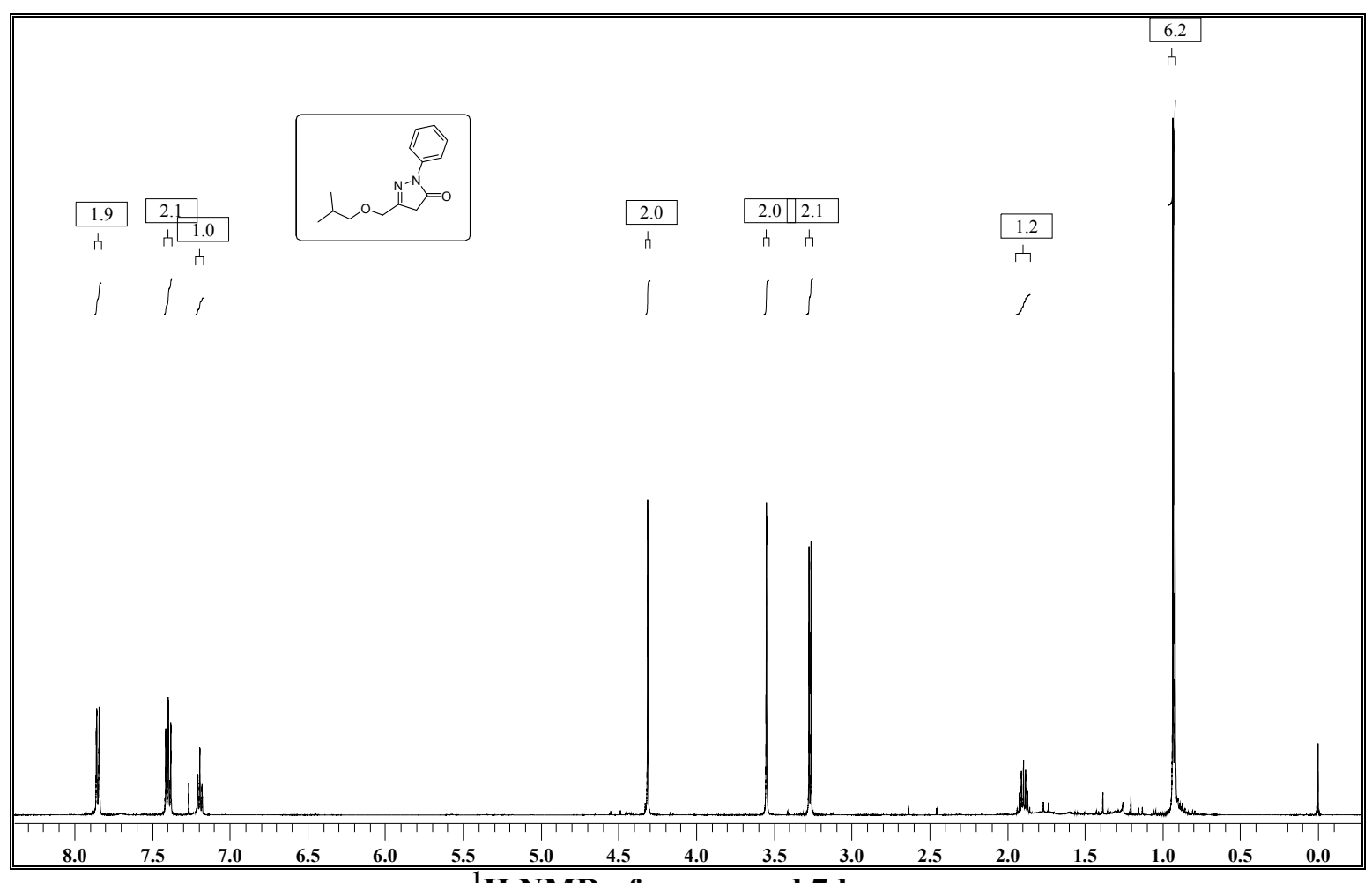

${ }^{1} \mathrm{H}$ NMR of compound 7d

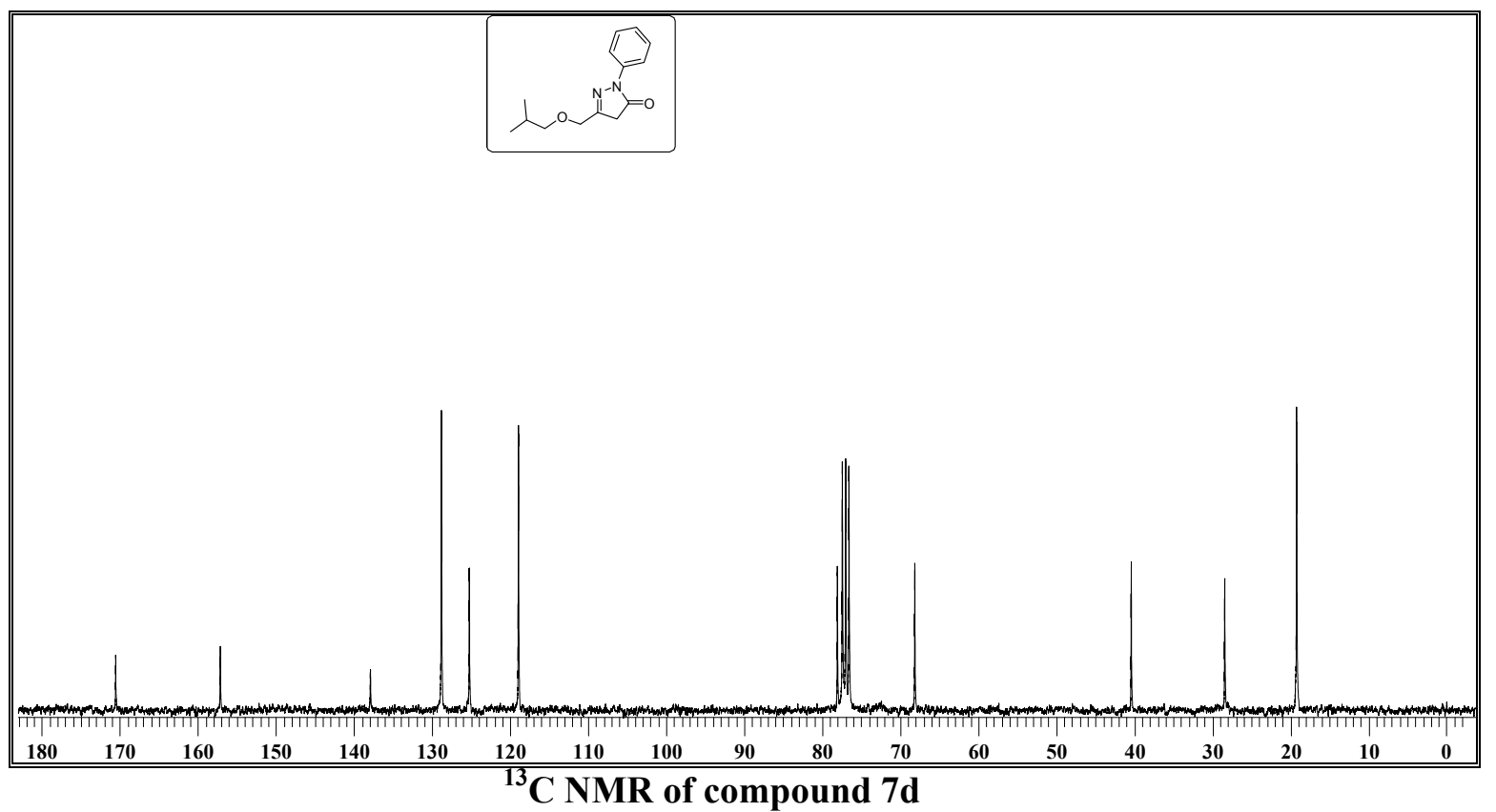




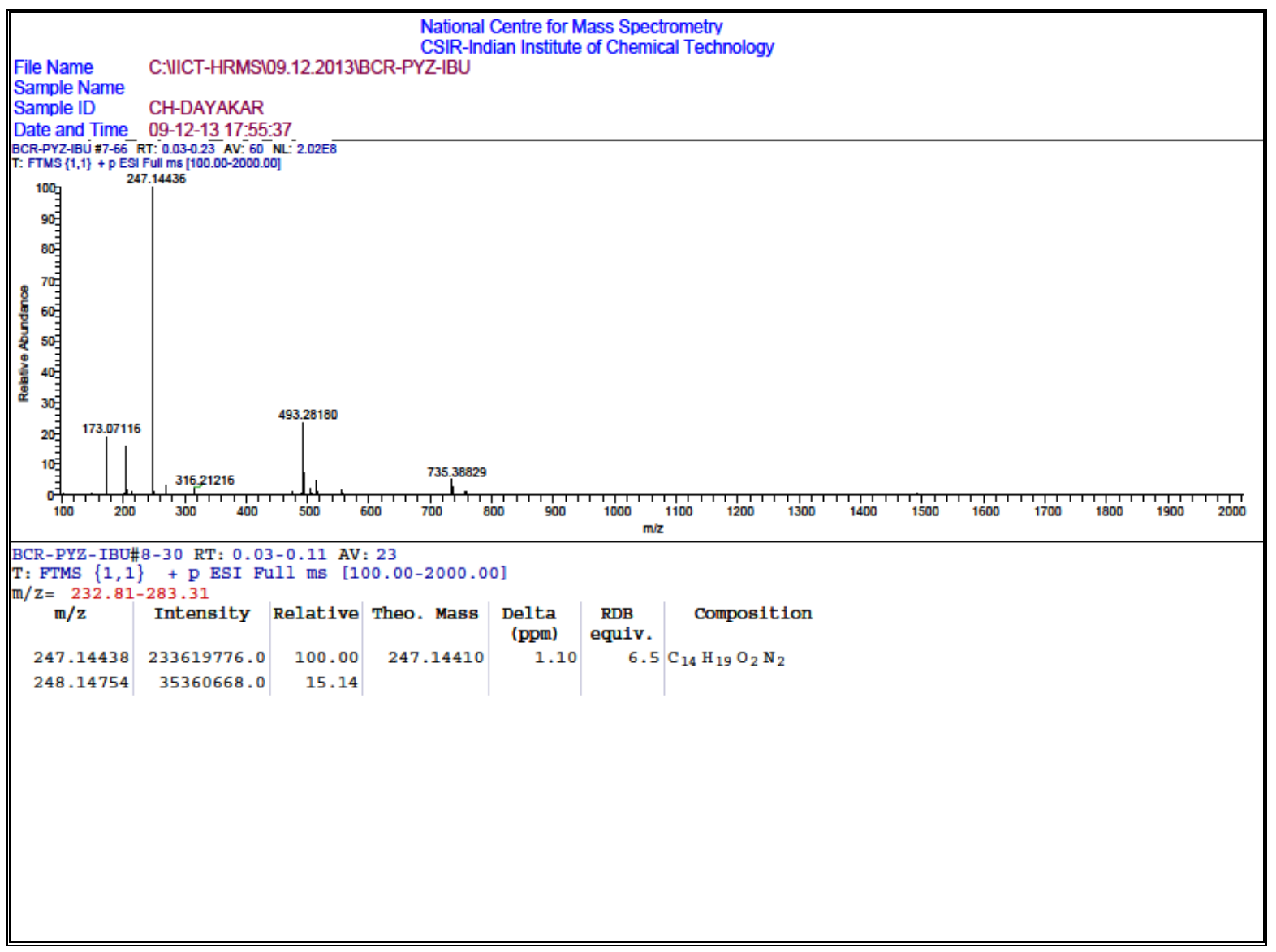

HRMS of compound 7d

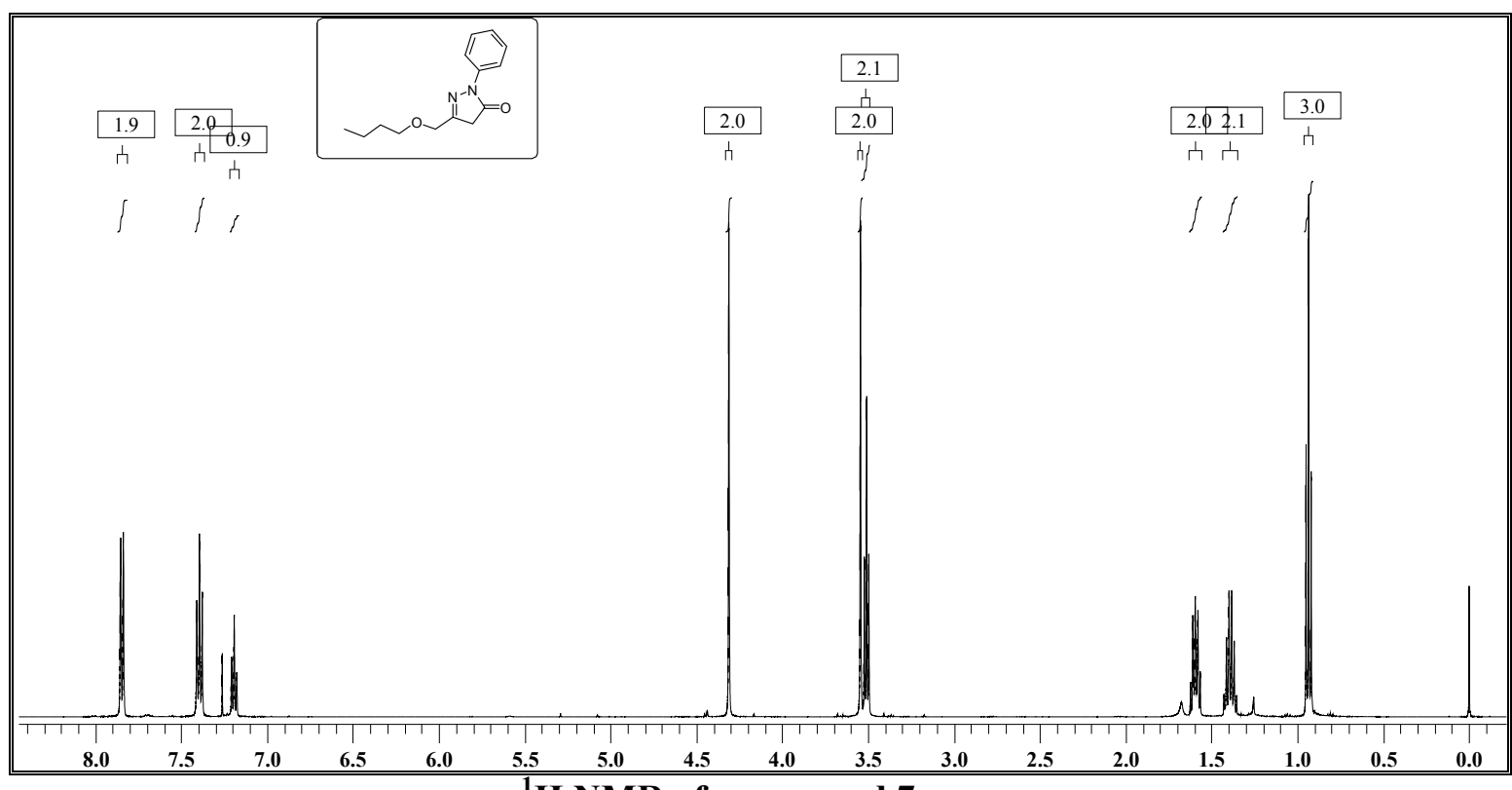

${ }^{1} \mathrm{H}$ NMR of compound $7 \mathrm{e}$ 

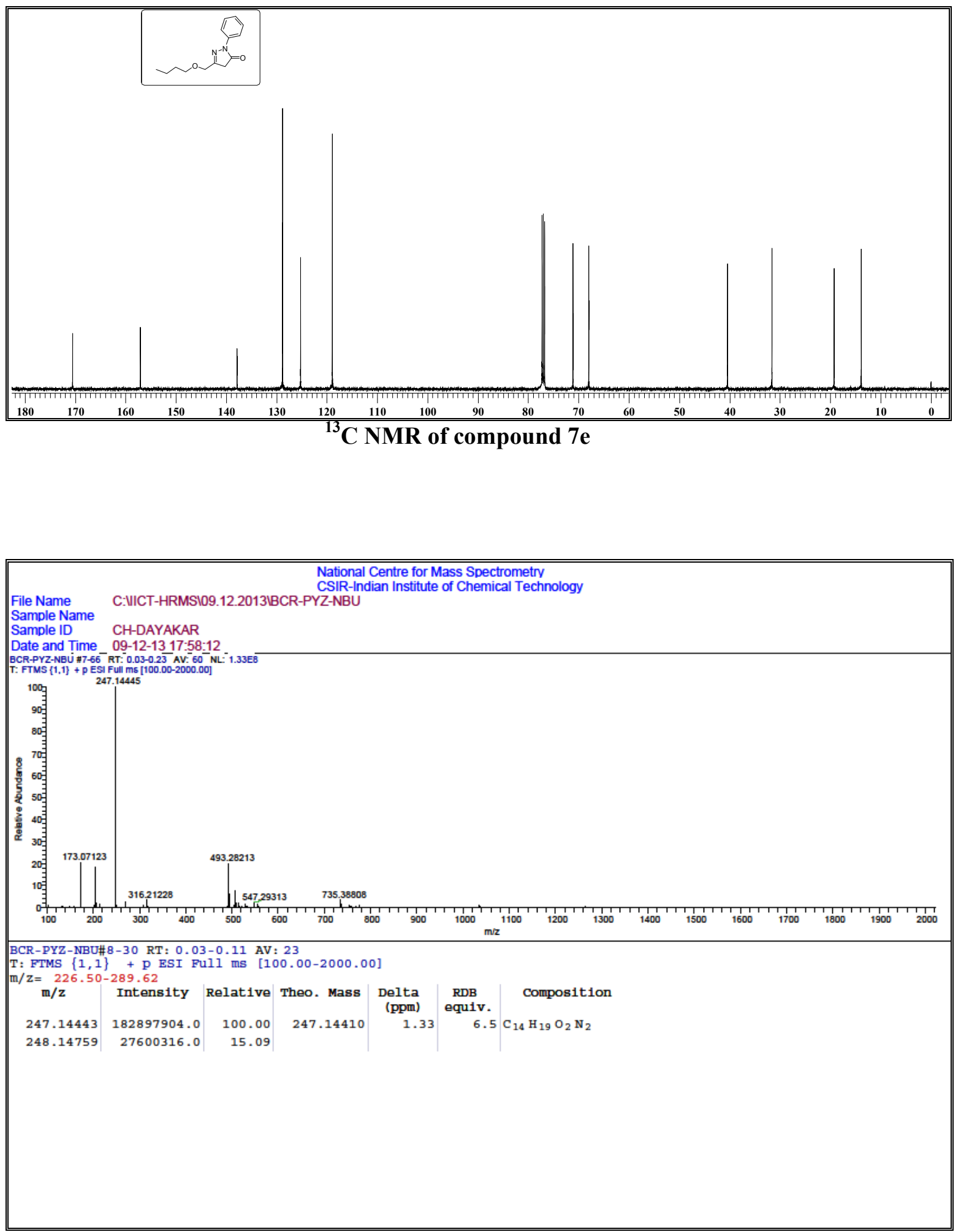

HRMS of compound 7e 

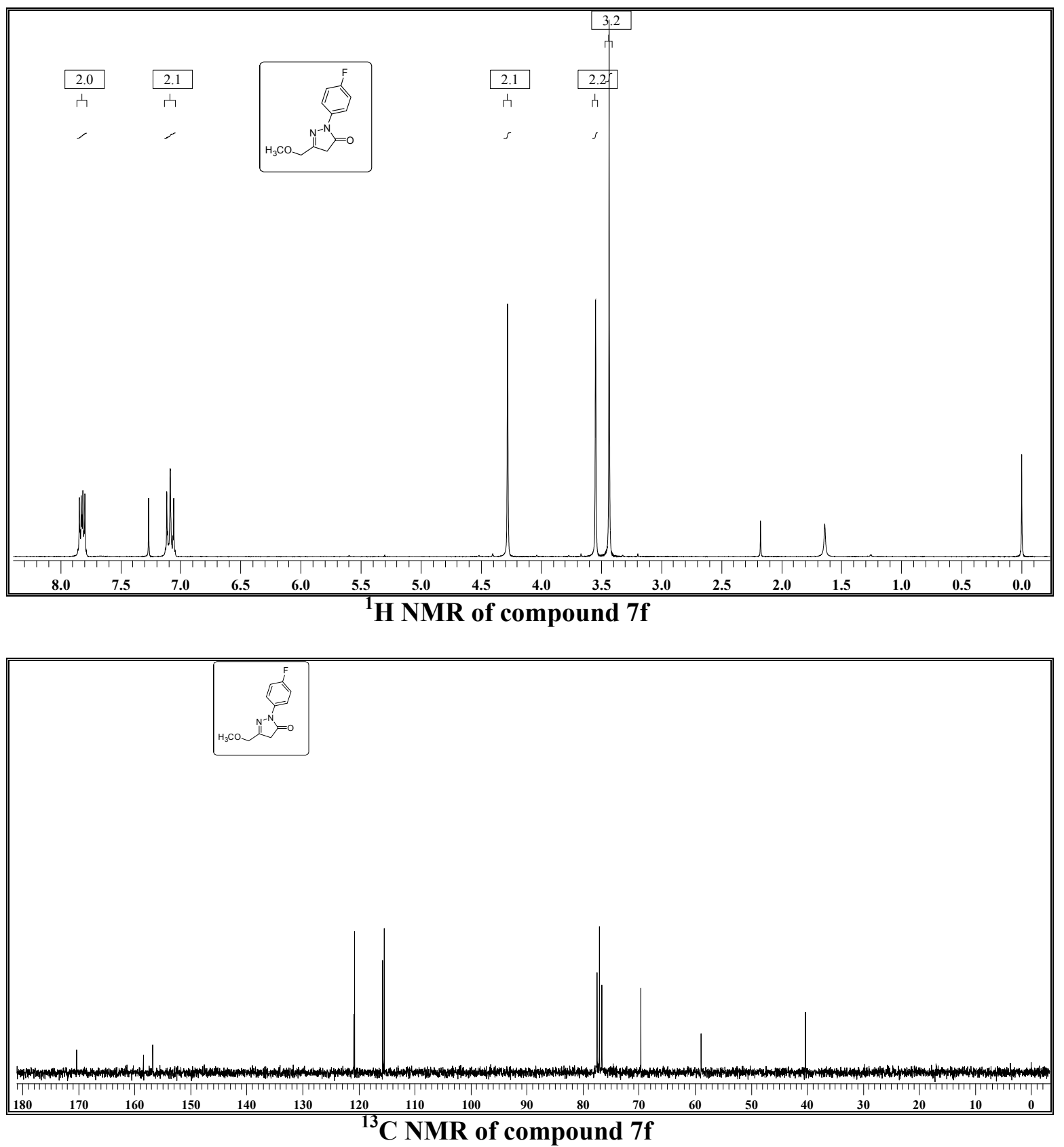


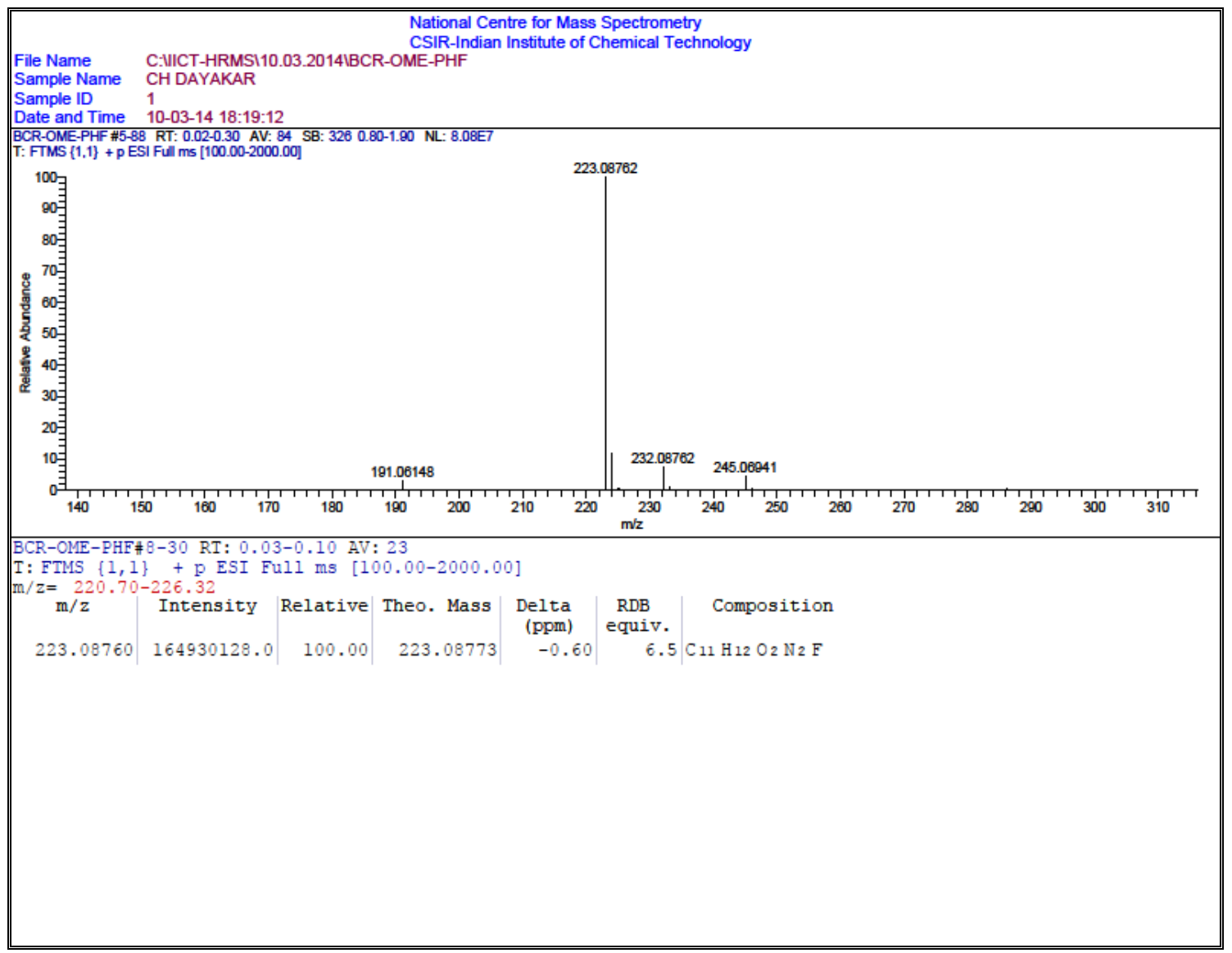

HRMS of compound 7f

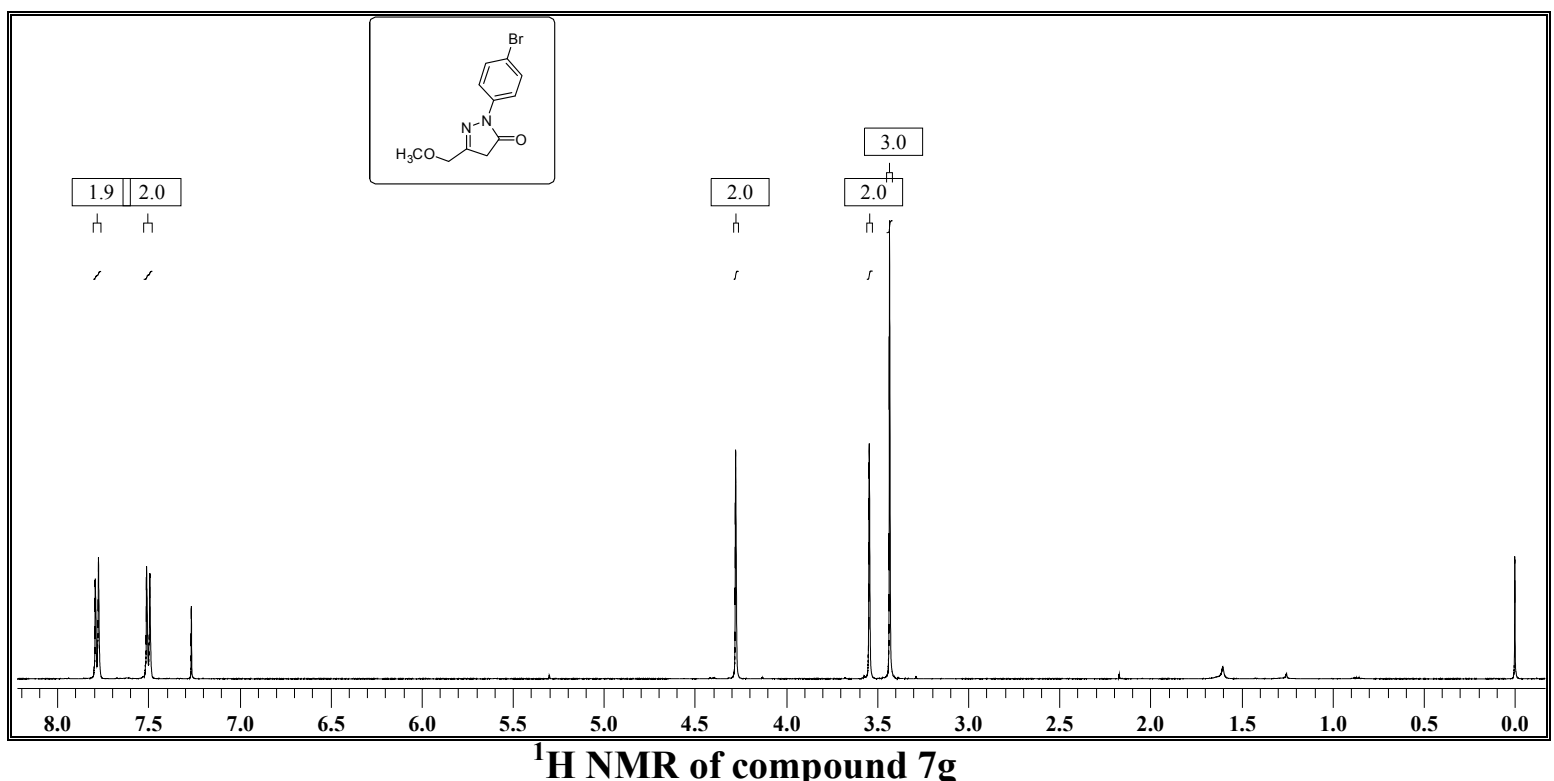



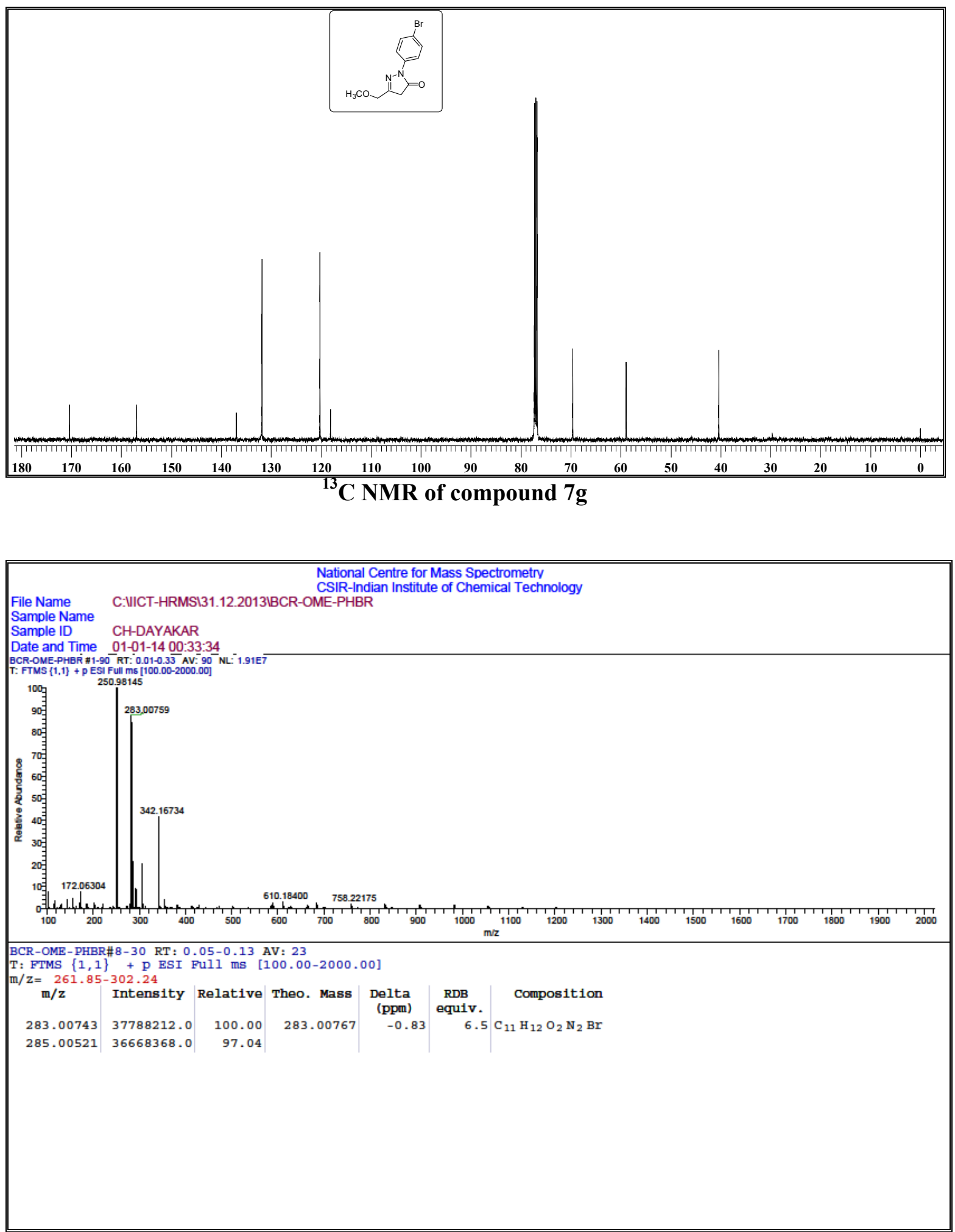

HRMS of compound $7 \mathrm{~g}$ 


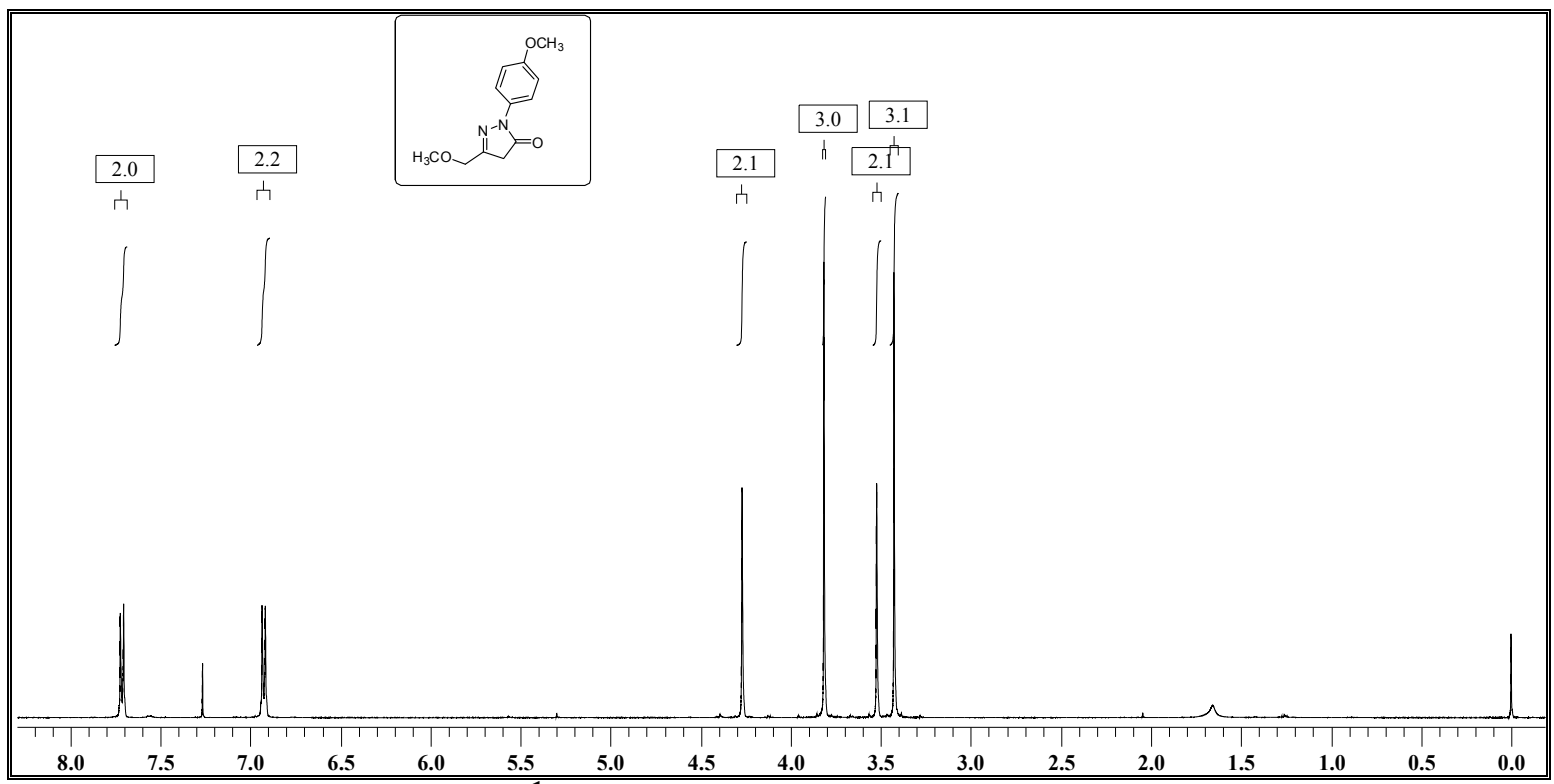

${ }^{1} \mathrm{H}$ NMR of compound $7 \mathrm{~h}$

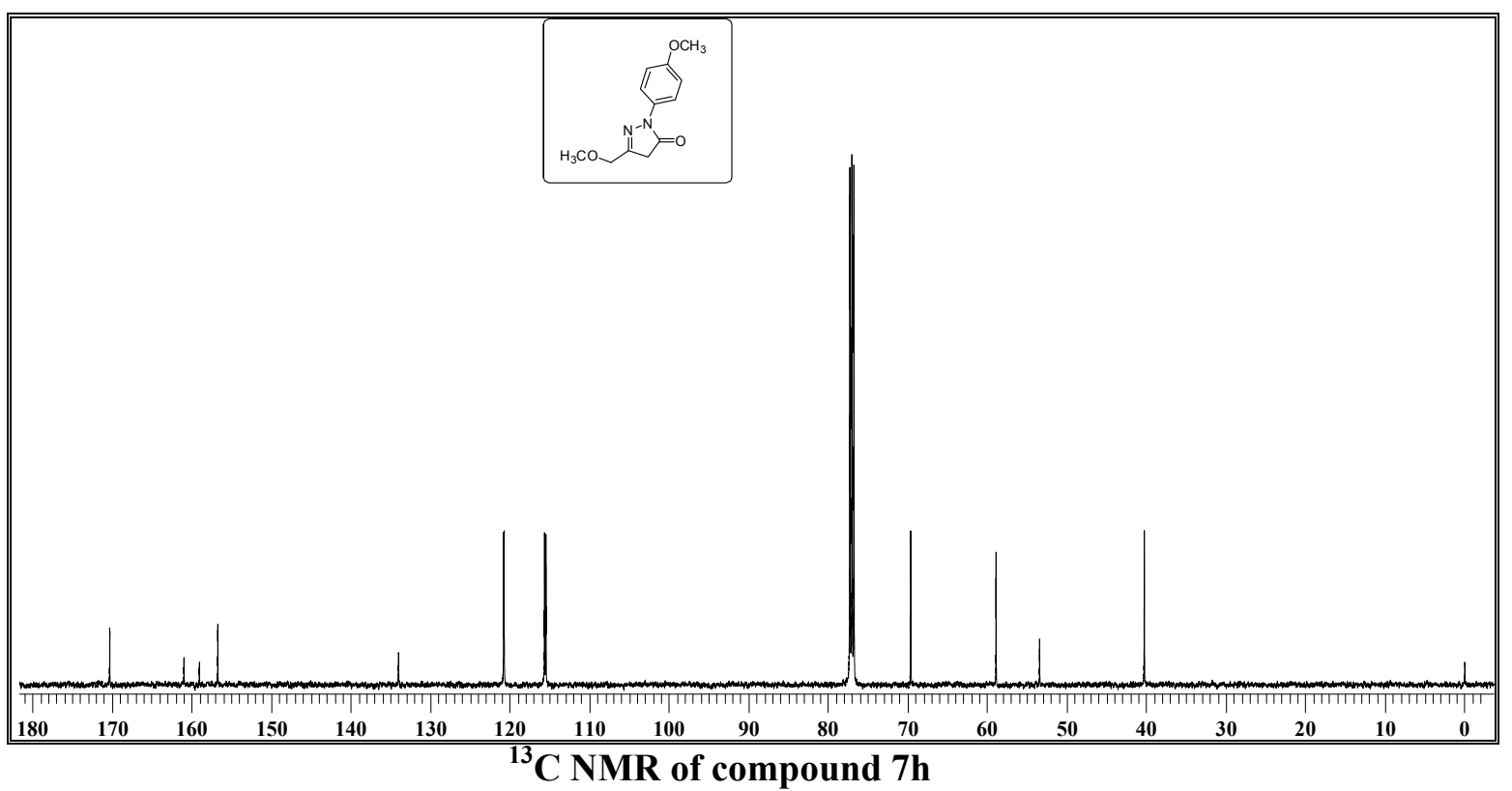




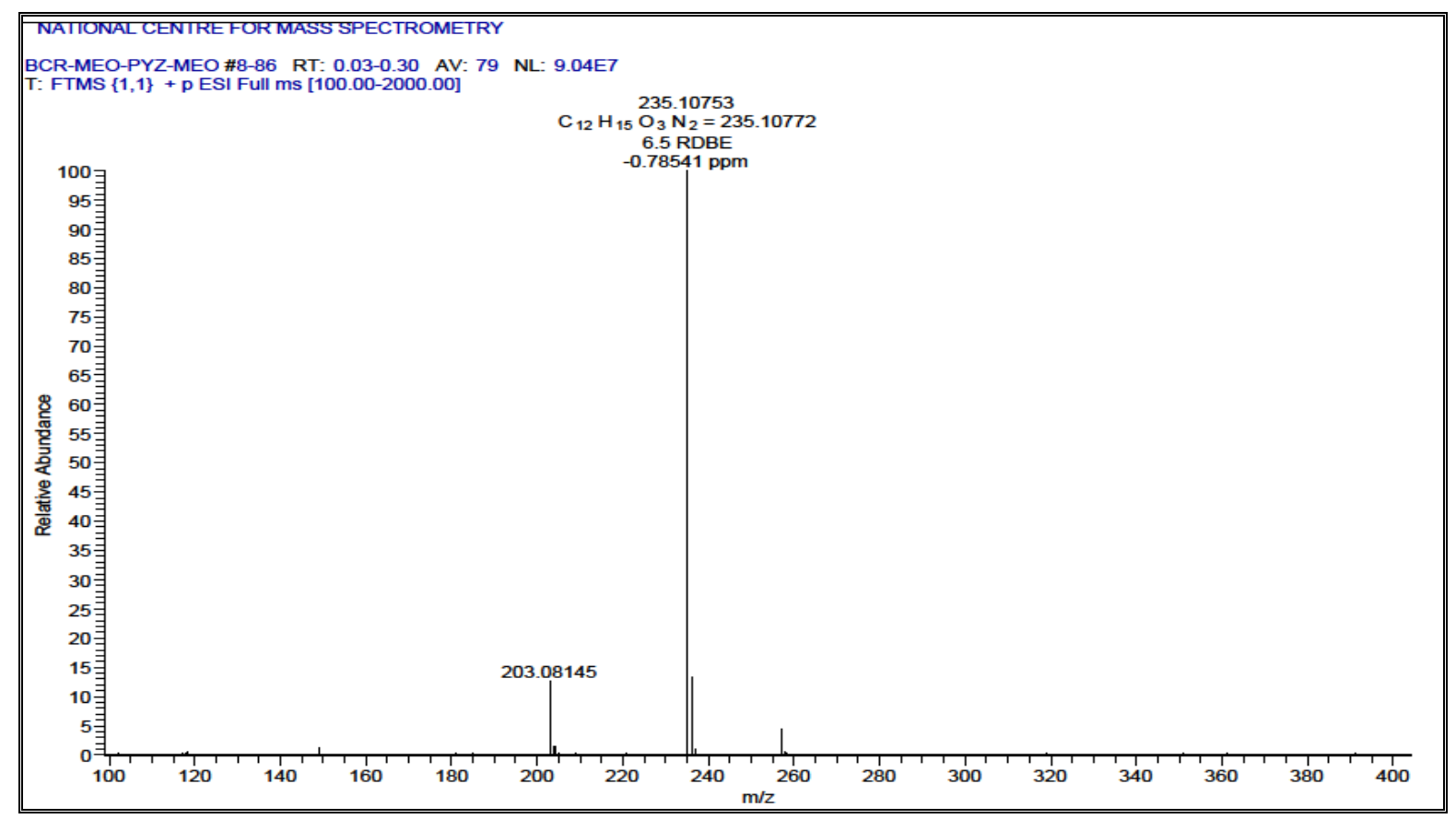

HRMS of compound $7 \mathrm{~h}$

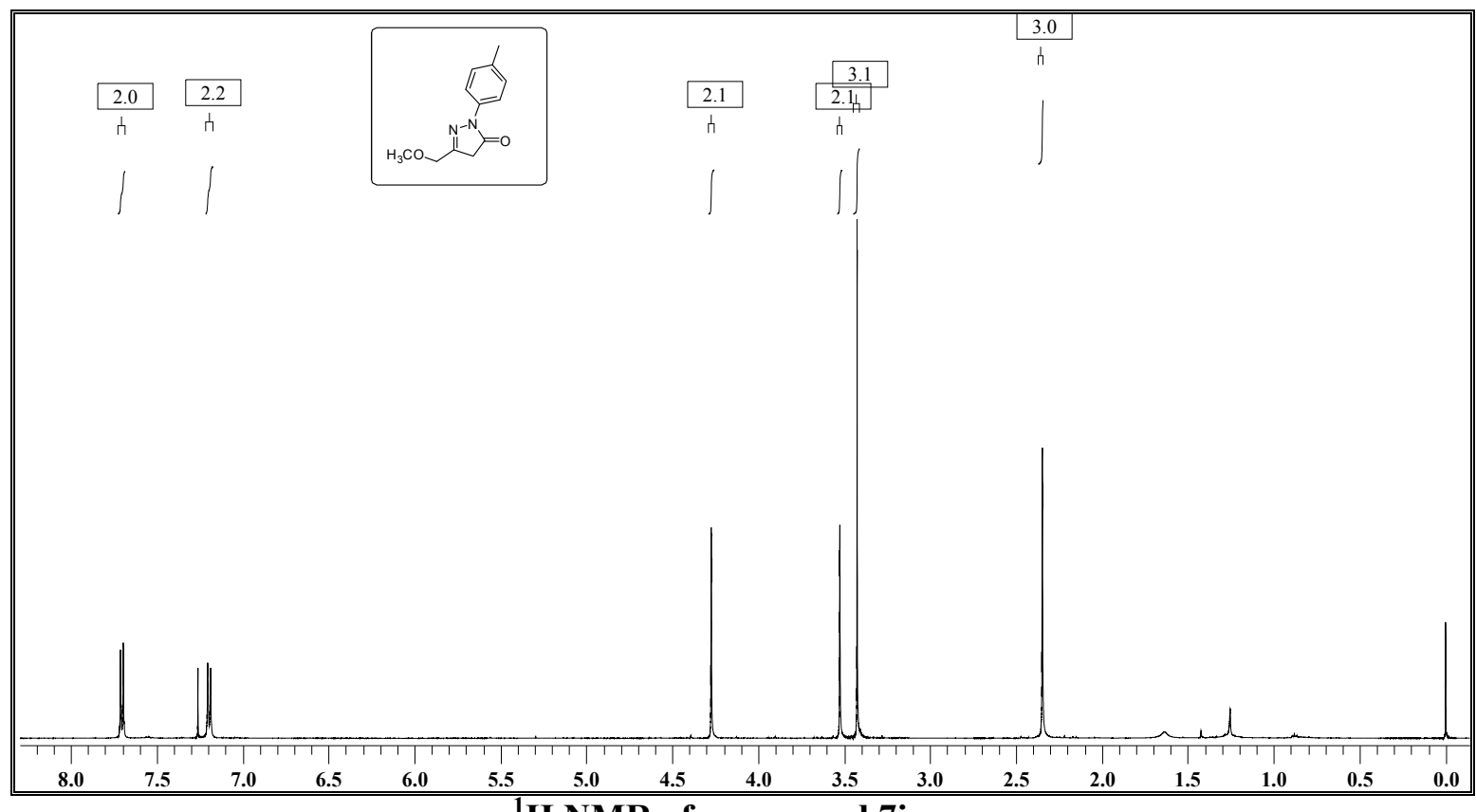

${ }^{1} H$ NMR of compound $7 i$ 

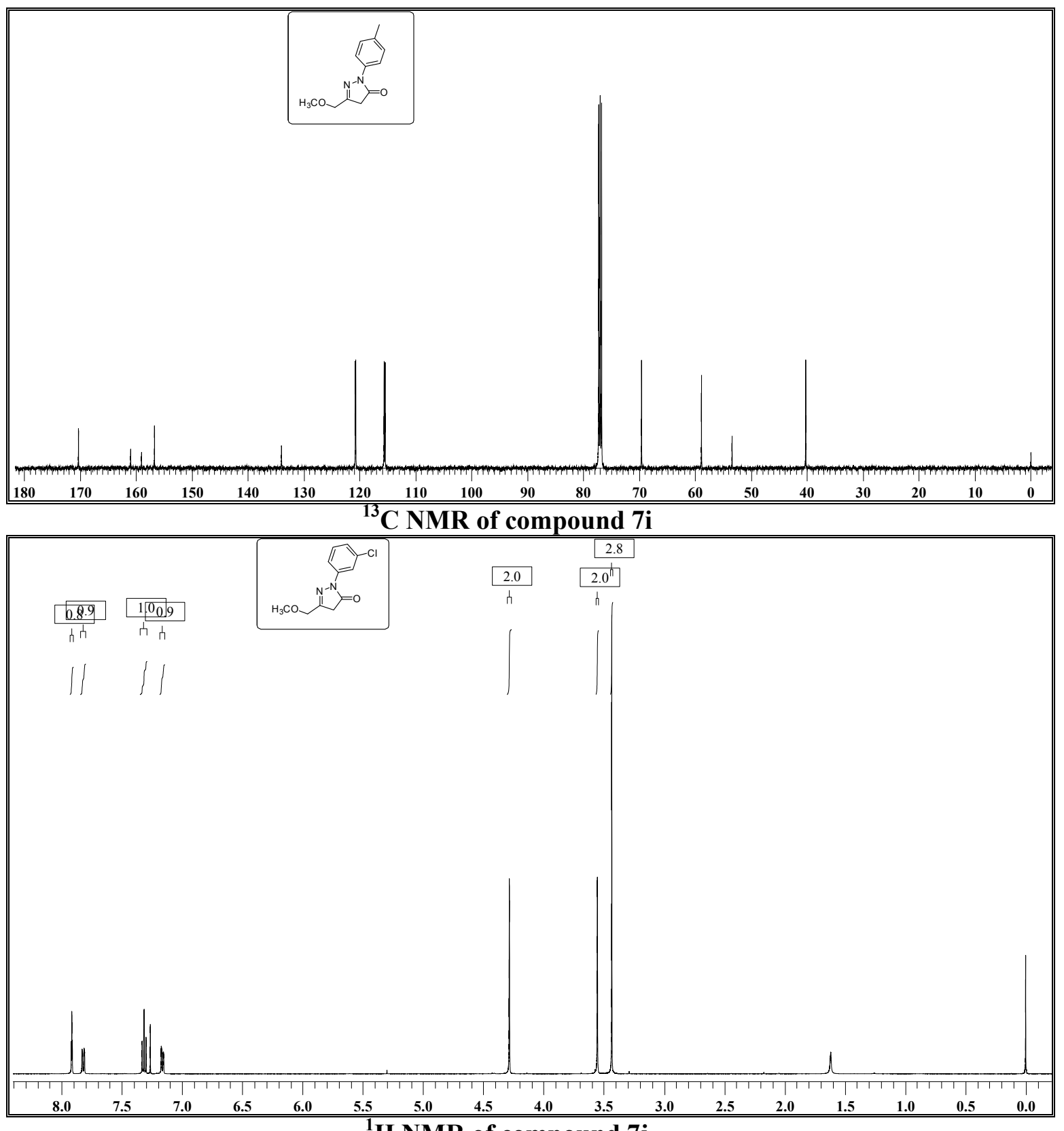

${ }^{1} \mathrm{H}$ NMR of compound $7 \mathbf{j}$ 

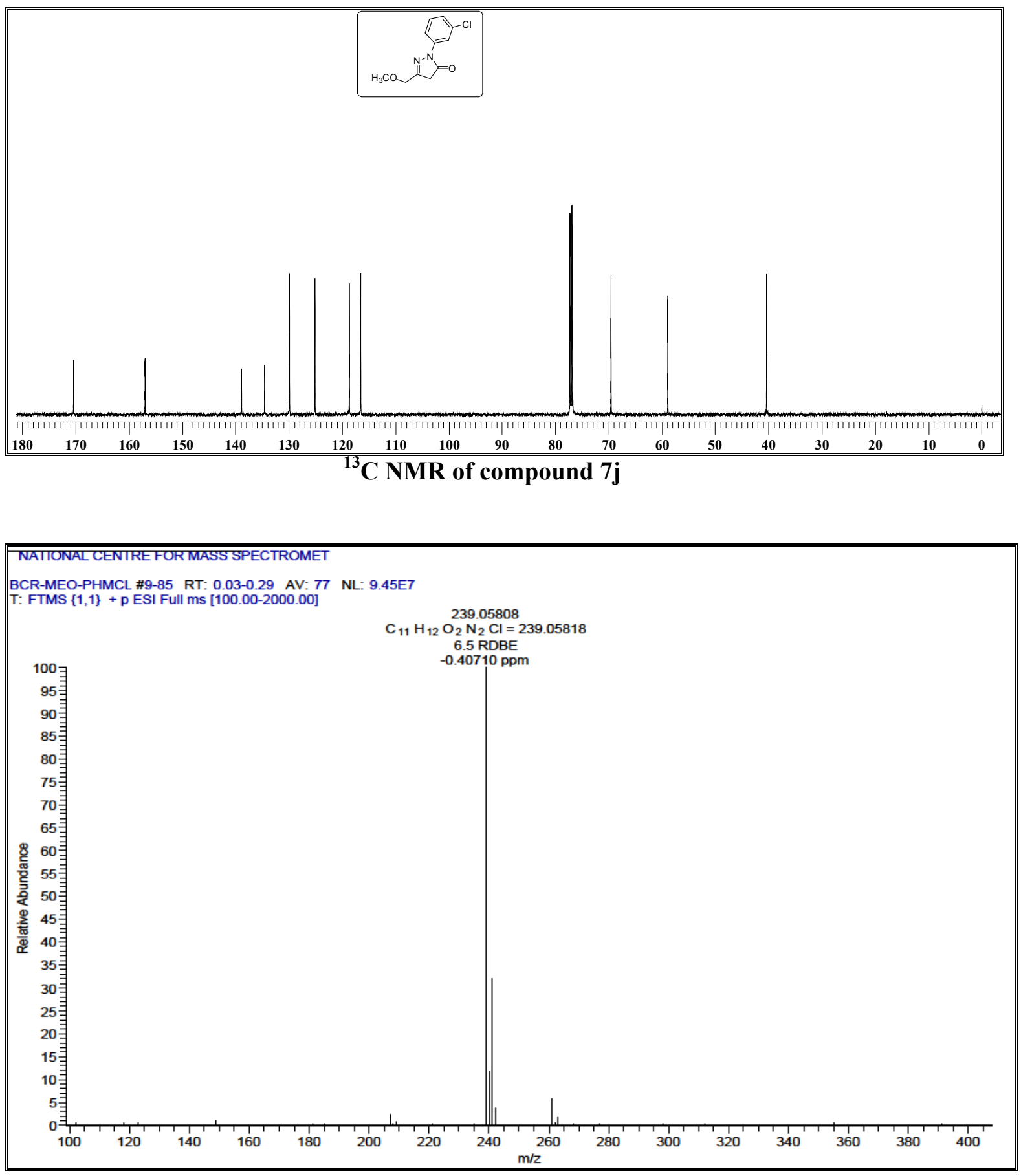

HRMS of compound 7j 


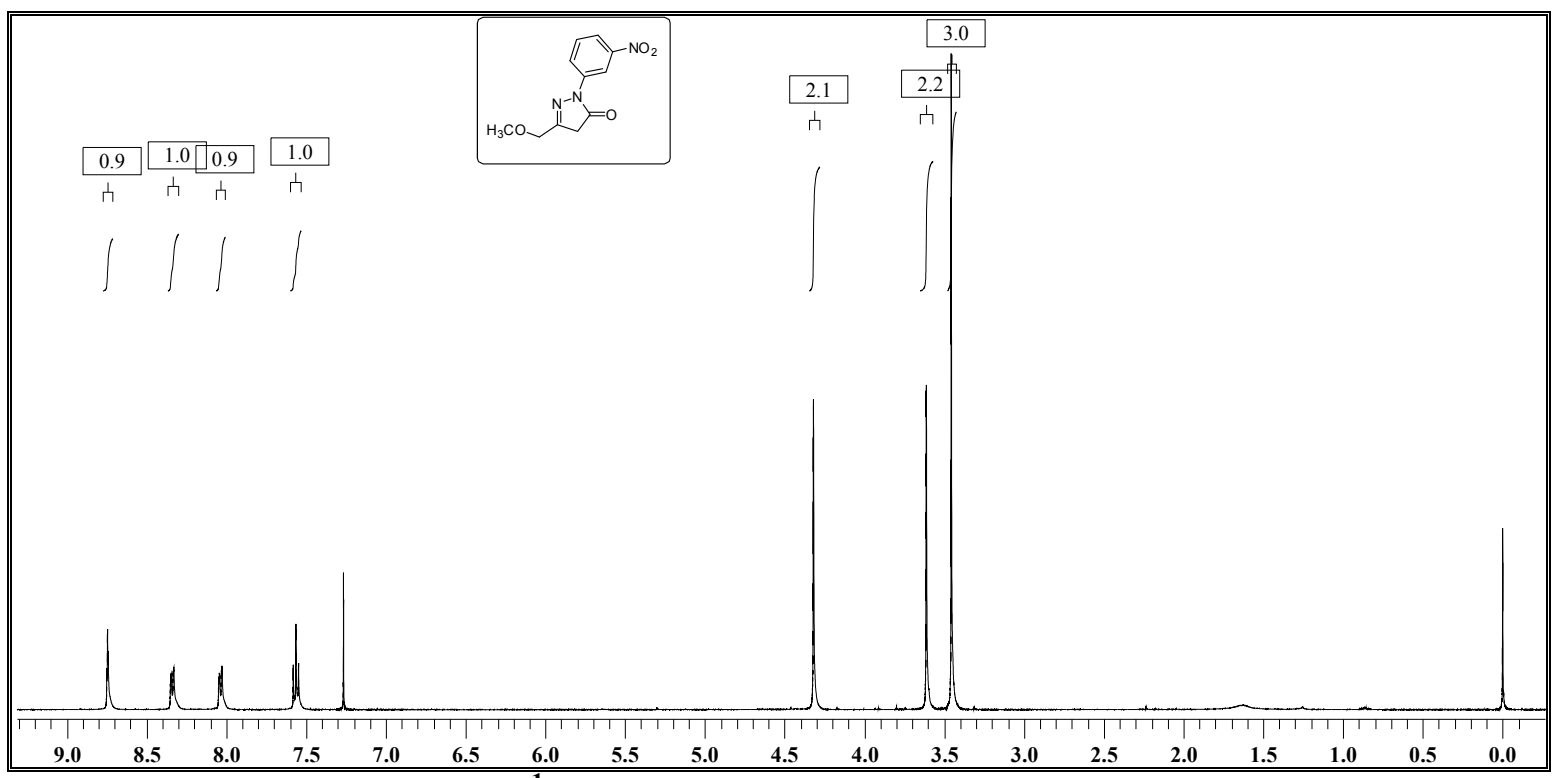

${ }^{1} H$ NMR of compound $7 k$

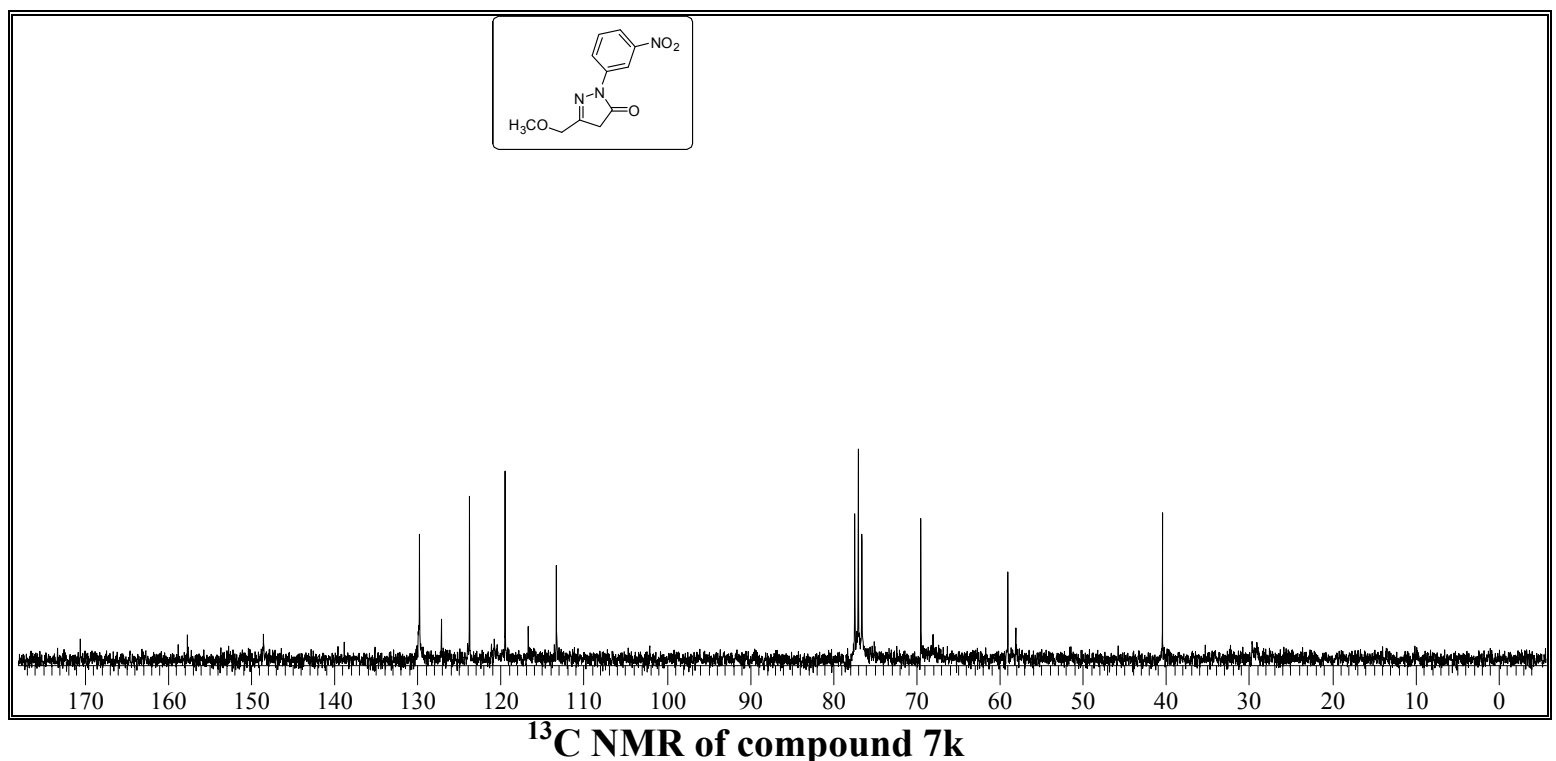




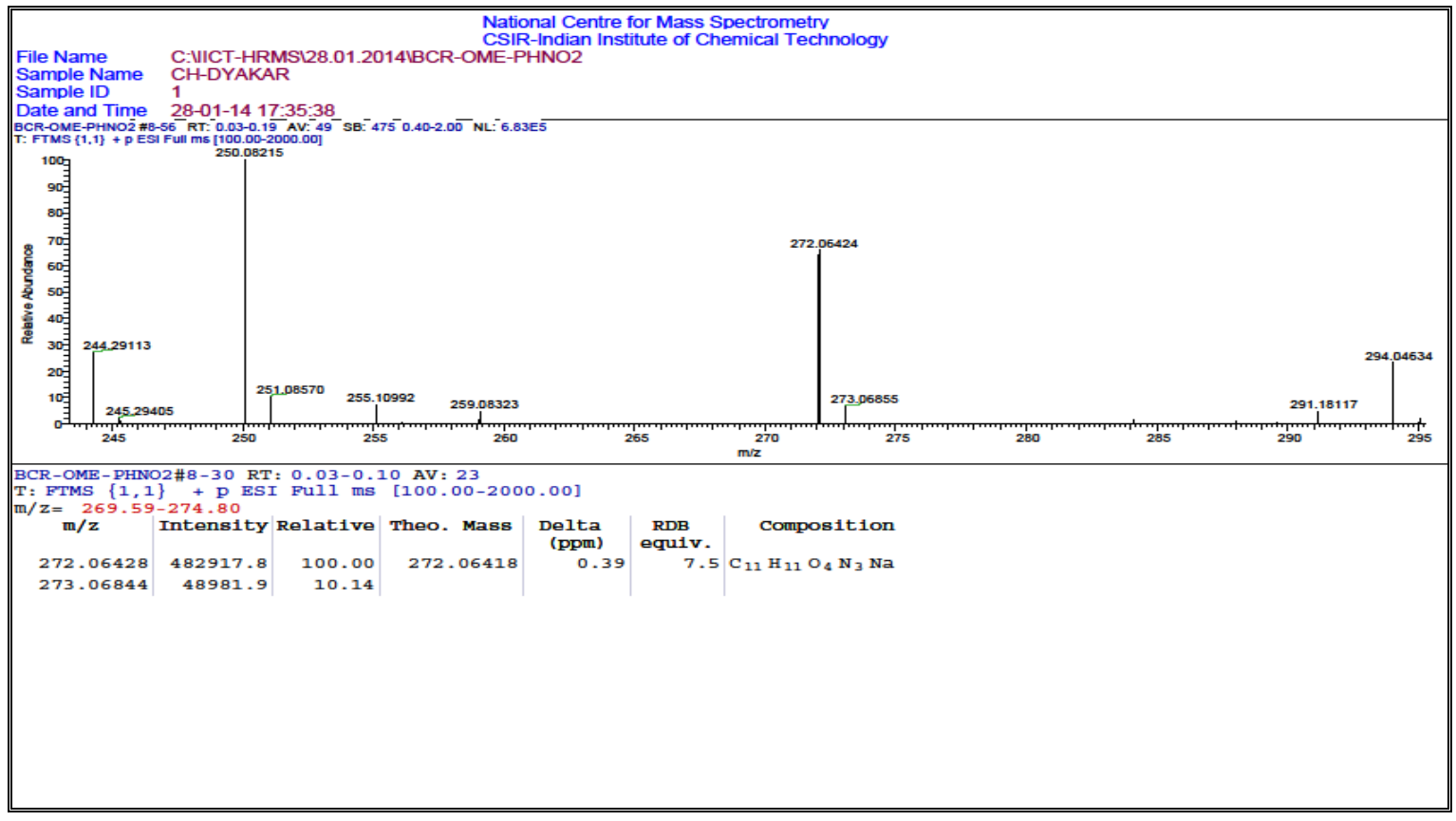

HRMS of compound $7 k$

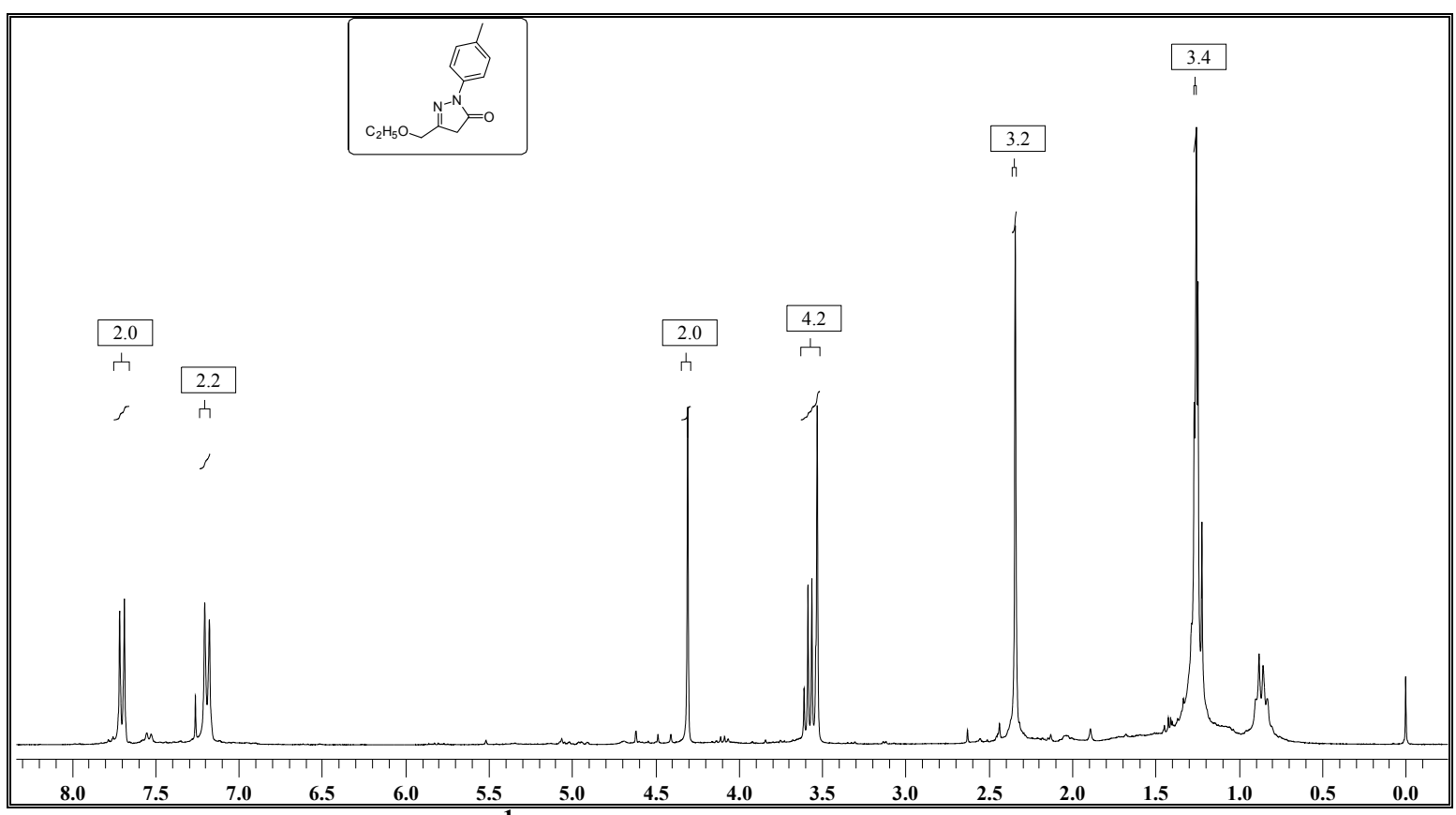

${ }^{1} H$ NMR of compound 71 


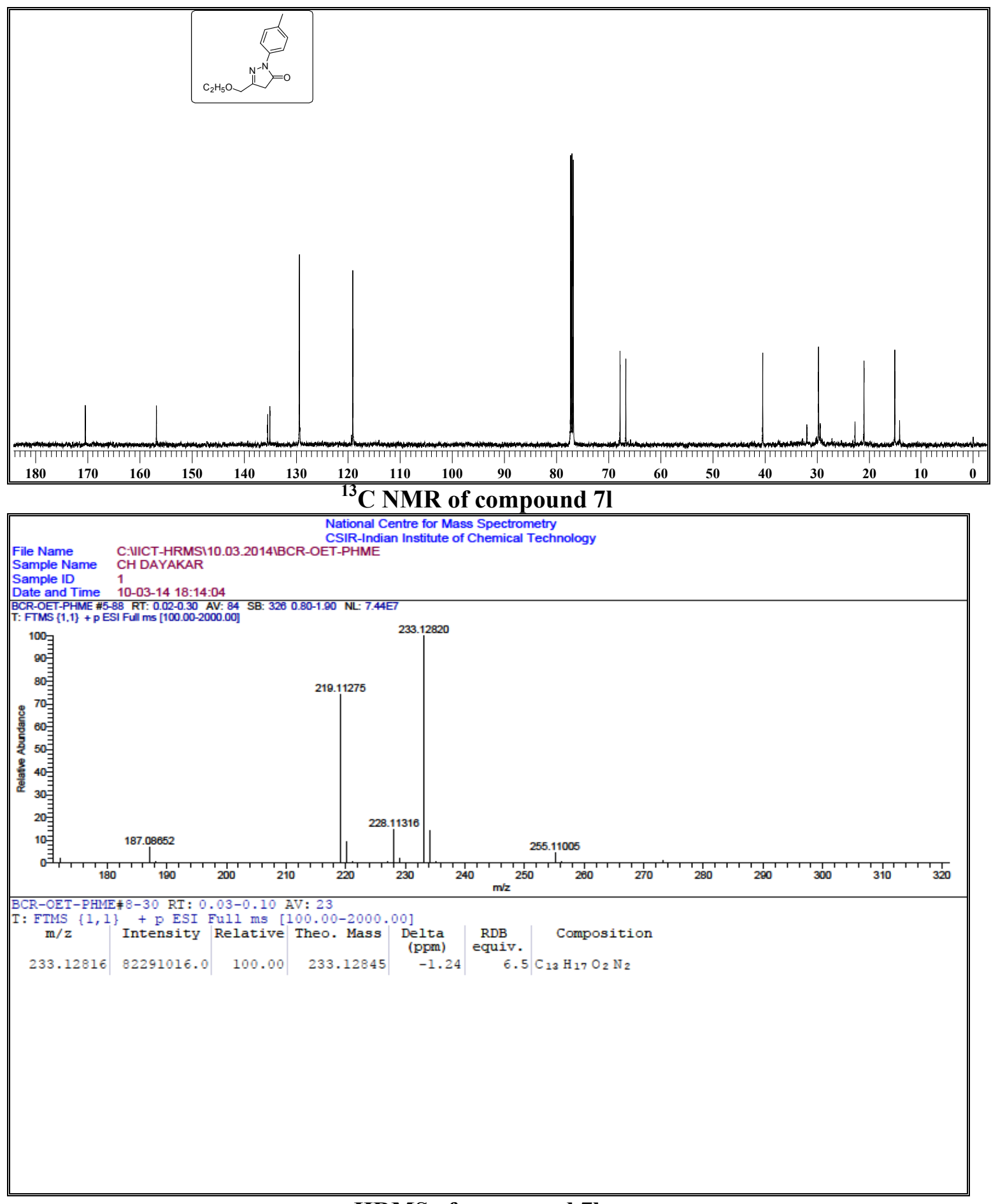

HRMS of compound 7l 


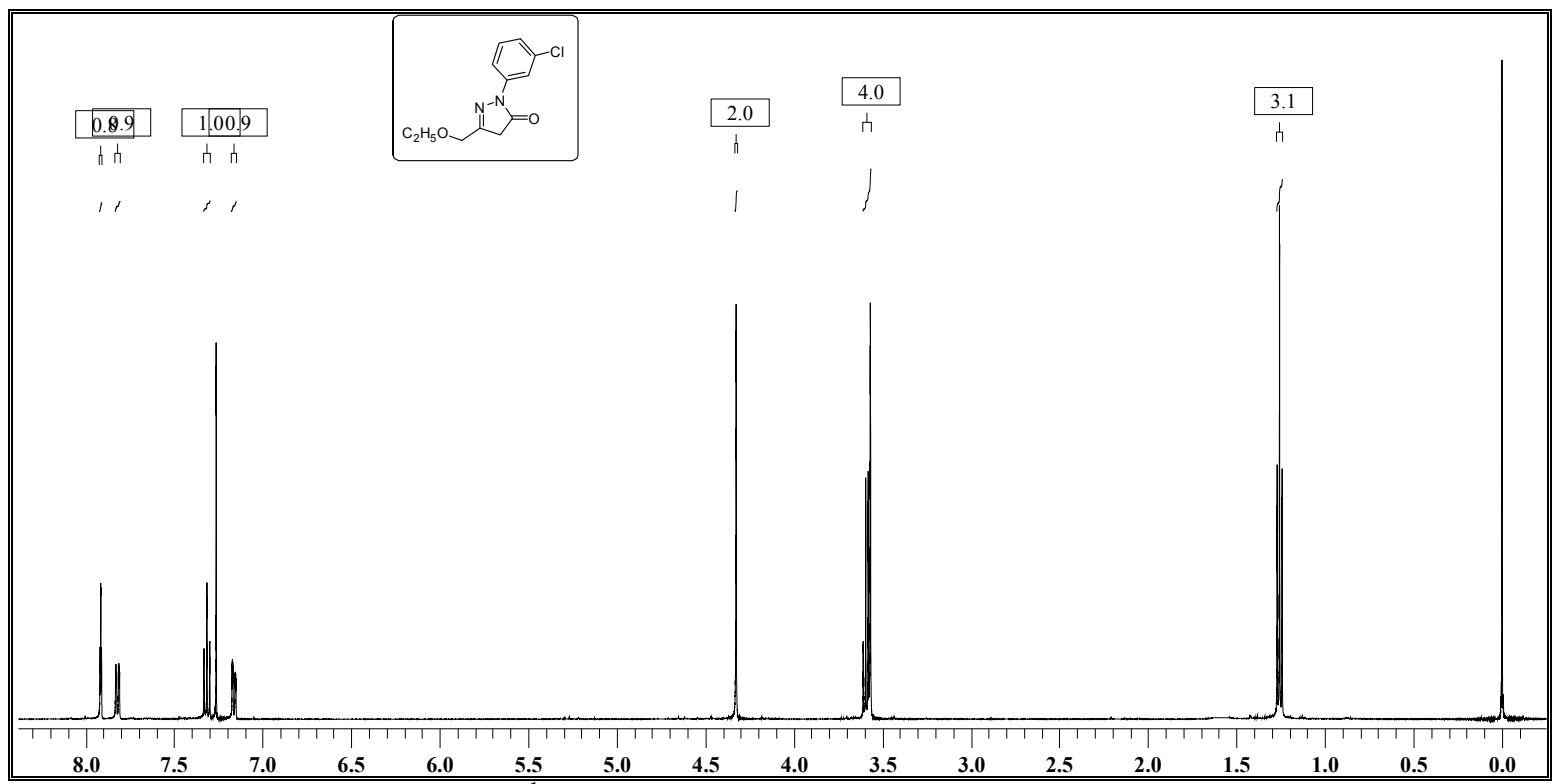

${ }^{1}$ H NMR of compound $7 \mathbf{m}$ 


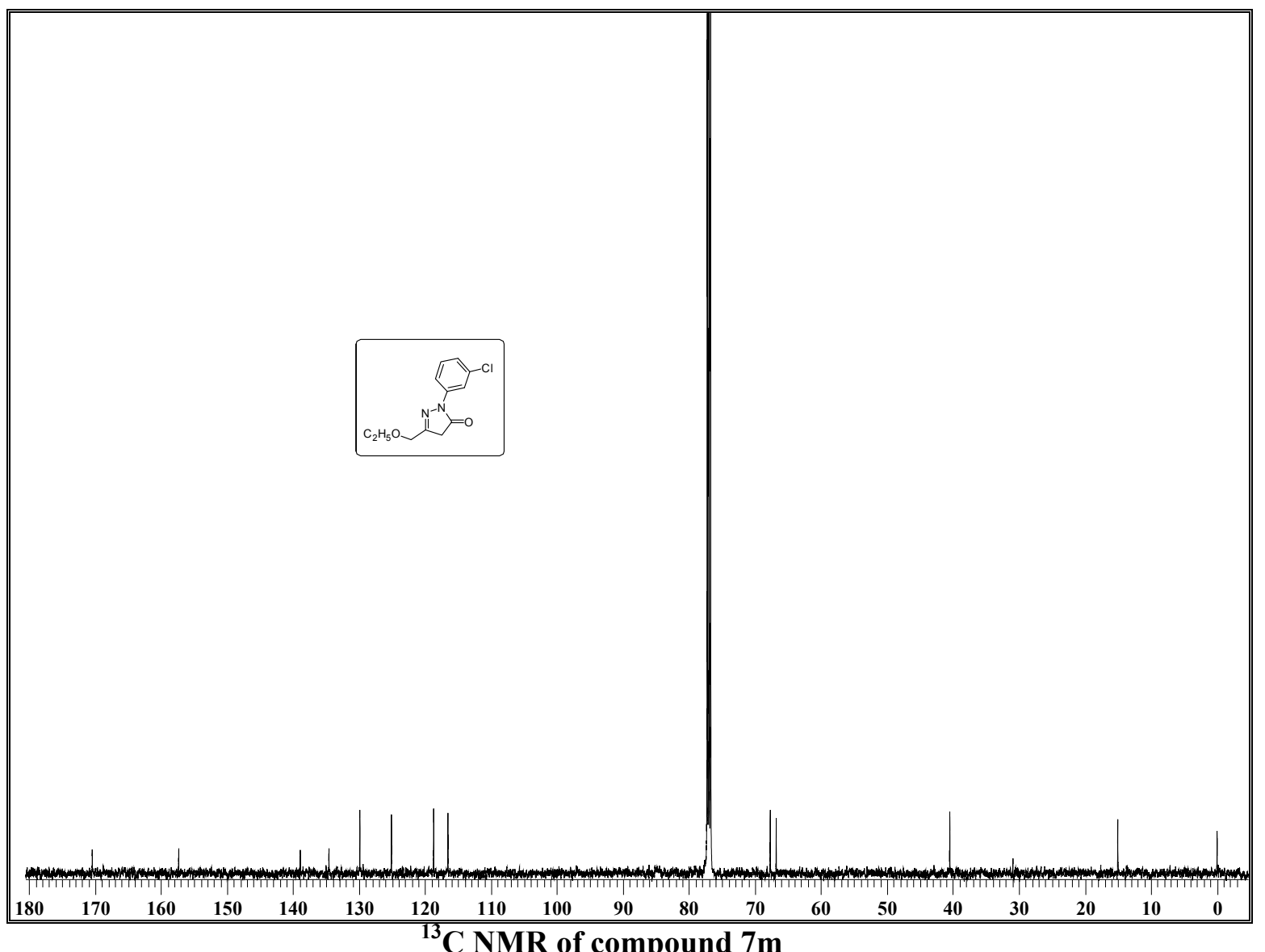




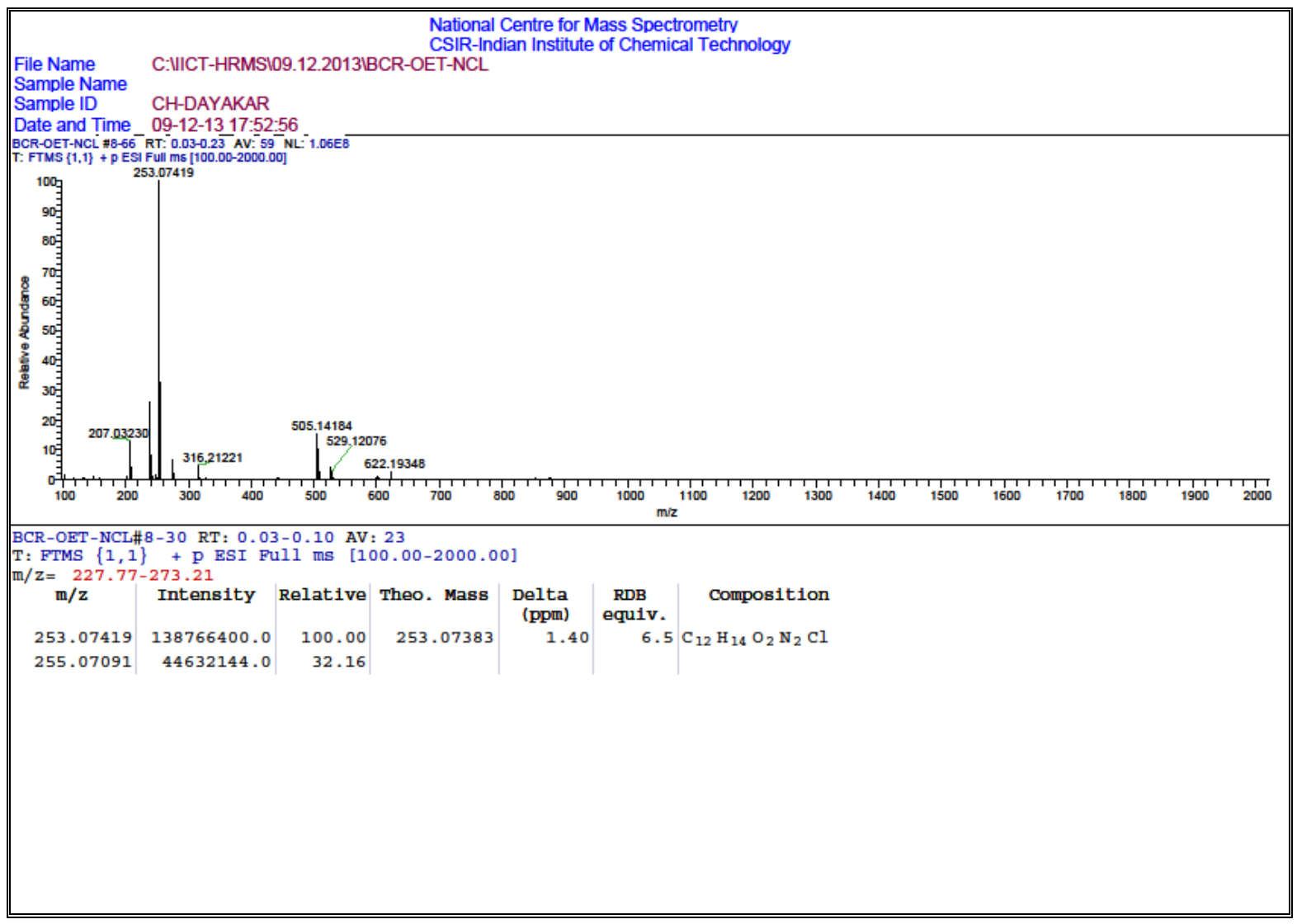

HRMS of compound 7m

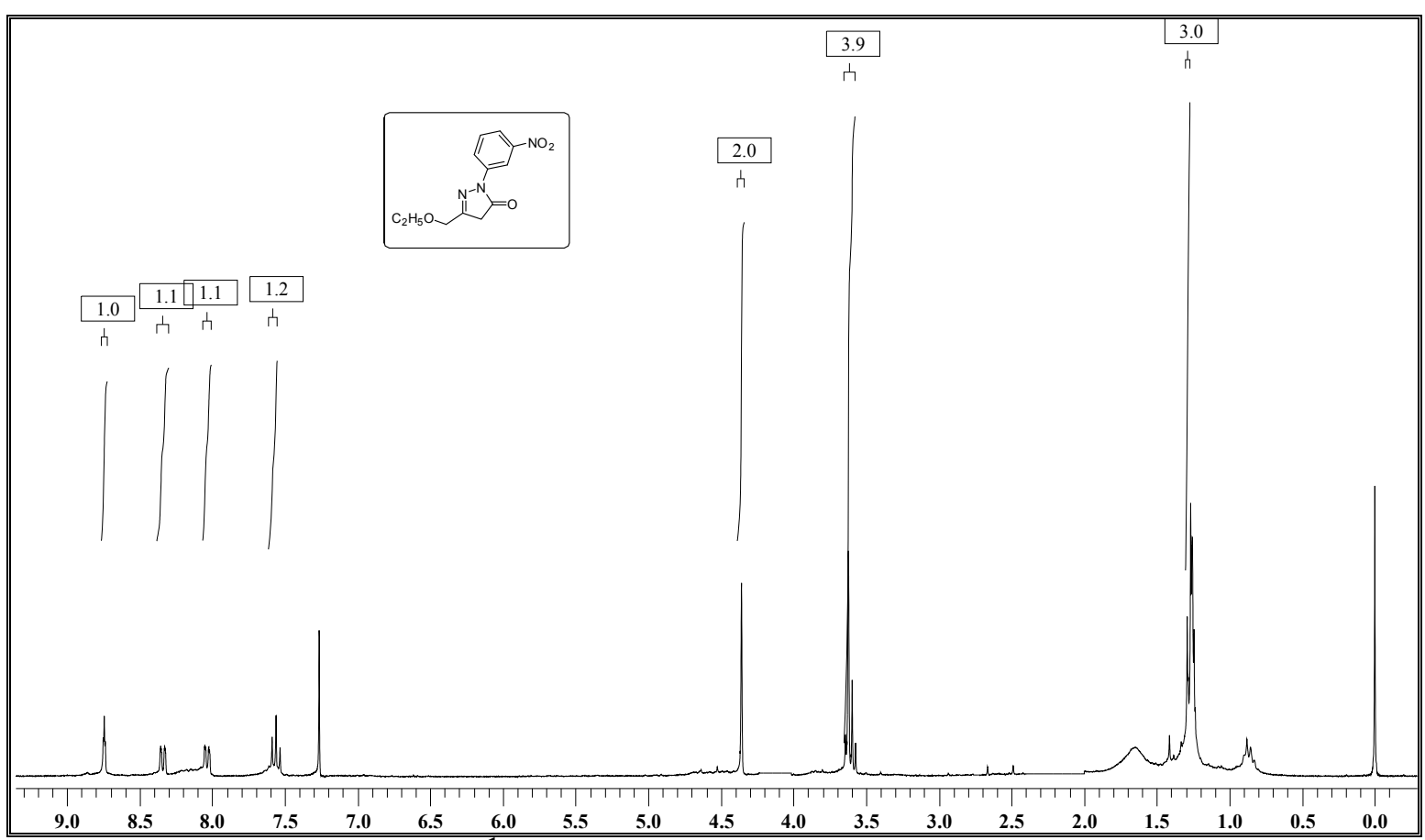

${ }^{1}$ H NMR of compound $7 \mathbf{n}$ 


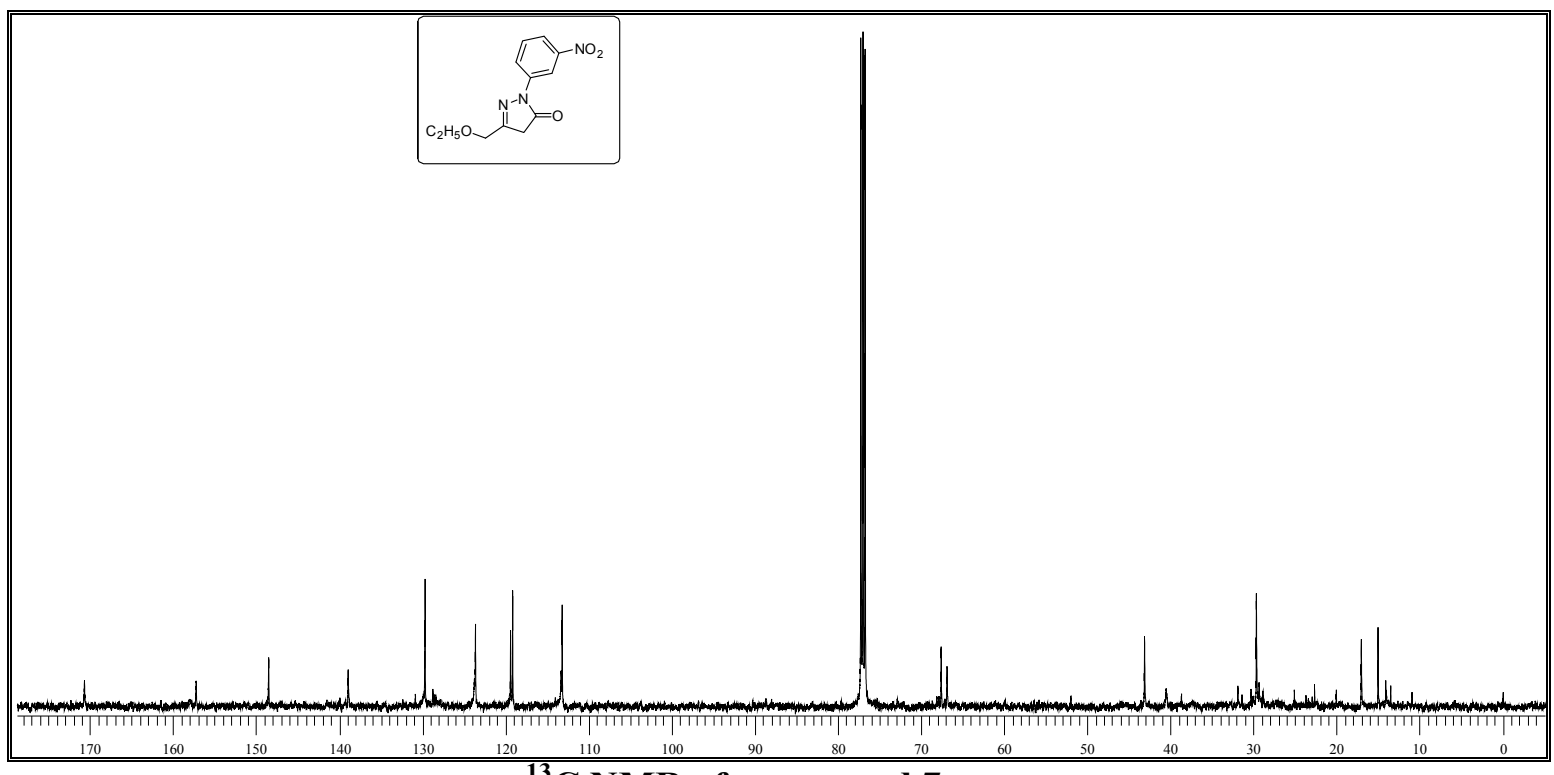

${ }^{13}$ C NMR of compound $7 n$

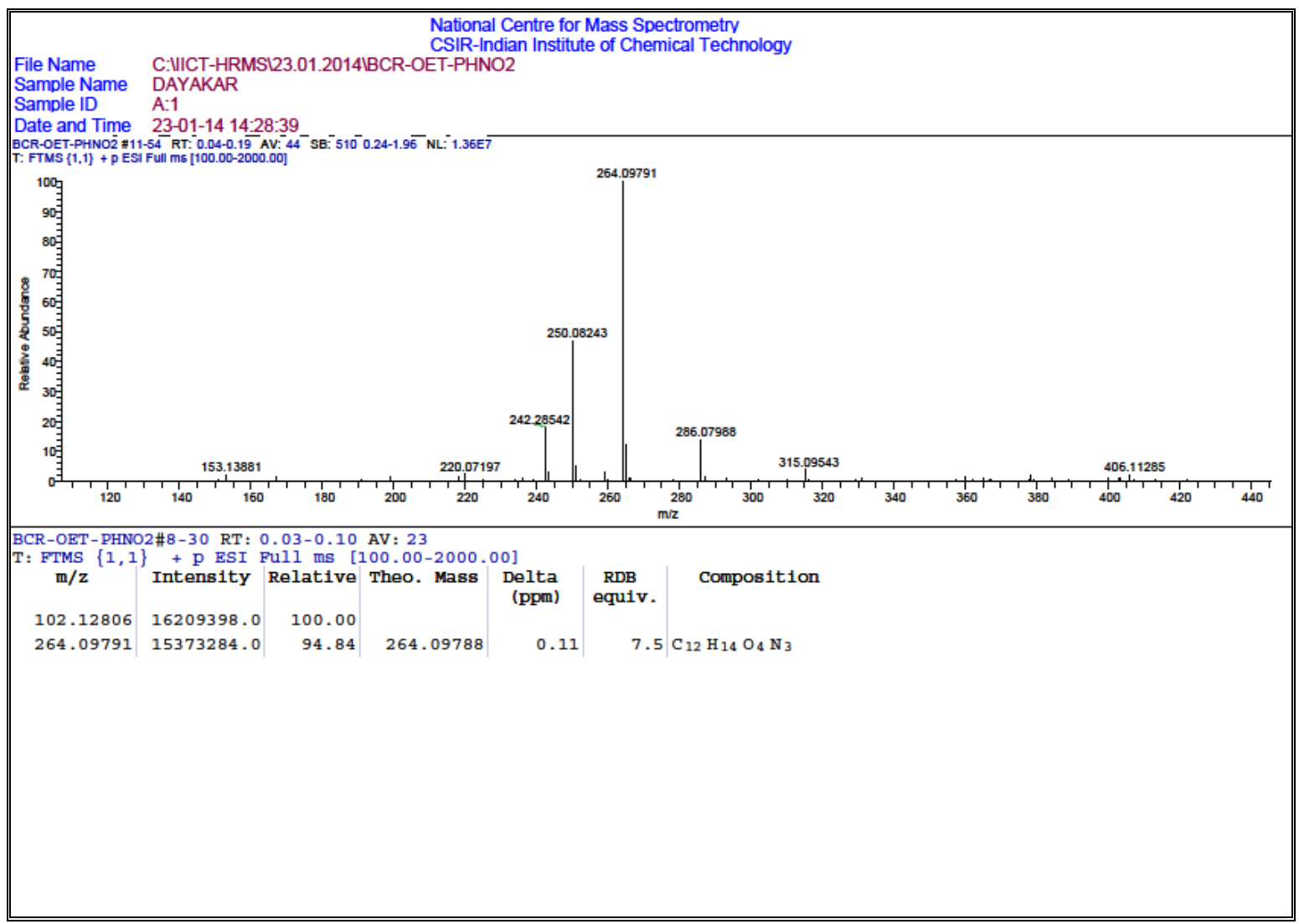

HRMS of compound $7 n$ 


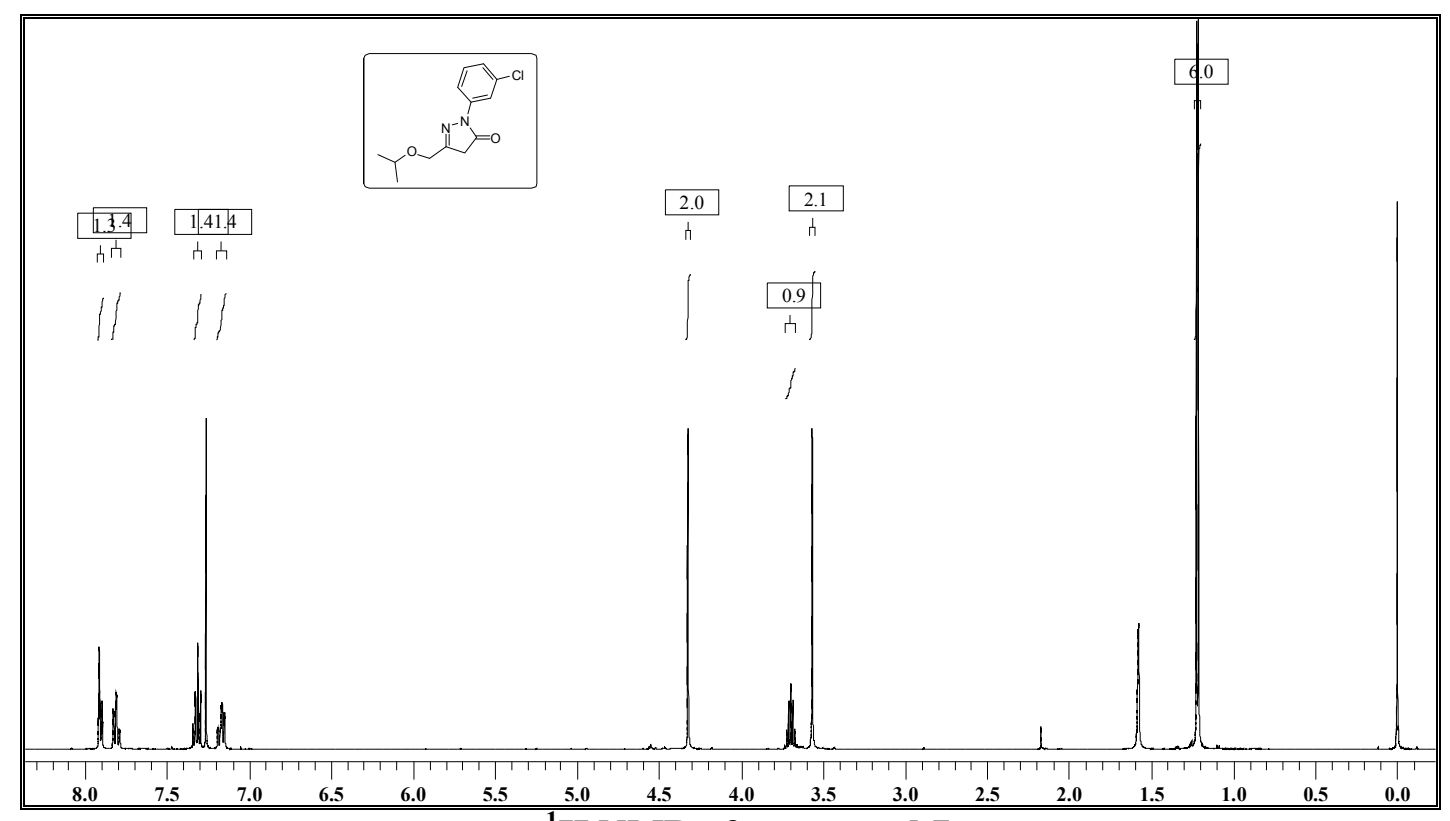

${ }^{1}$ H NMR of compound 7o 


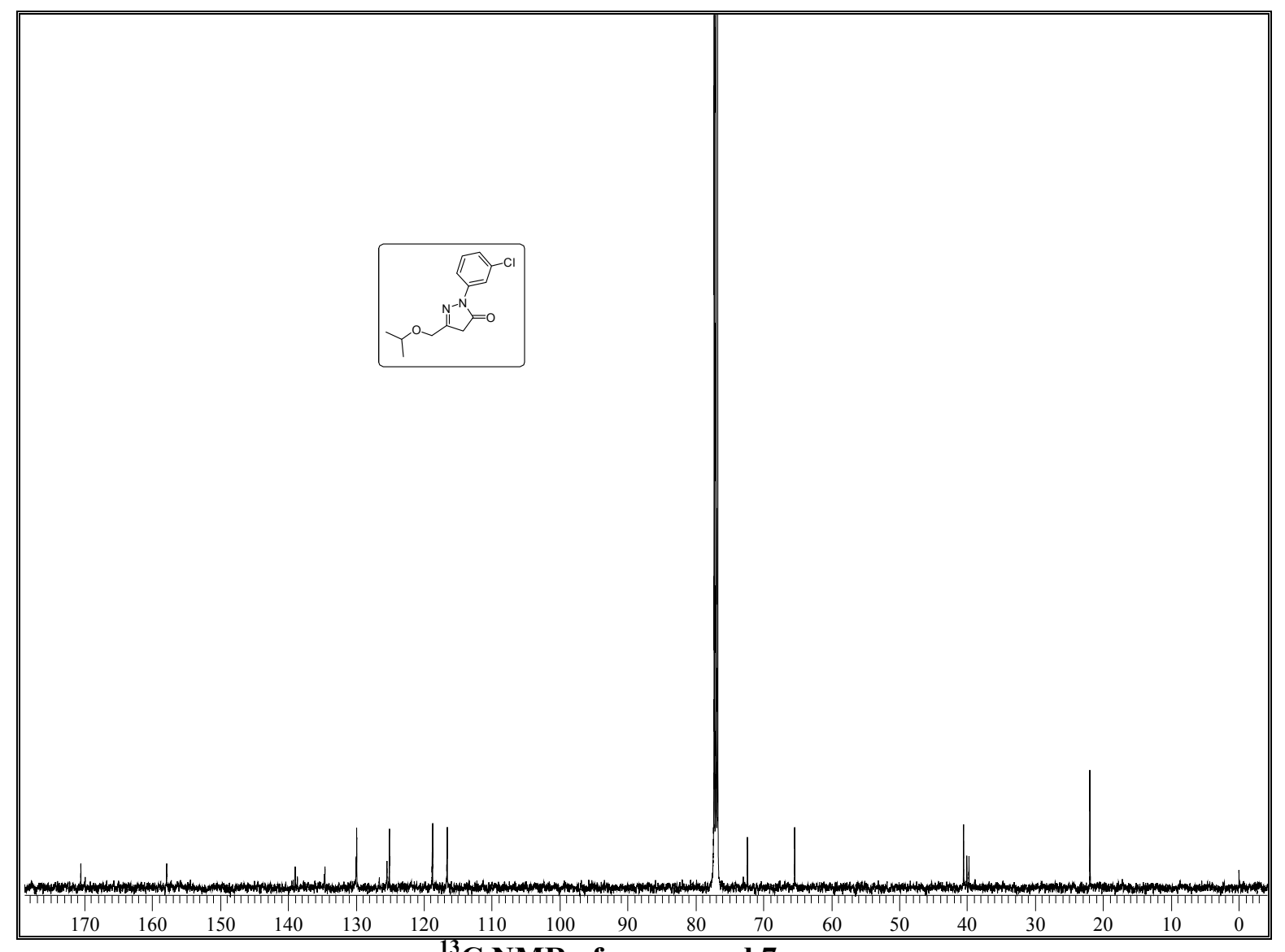

${ }^{13} \mathrm{C}$ NMR of compound $7 \mathrm{o}$ 


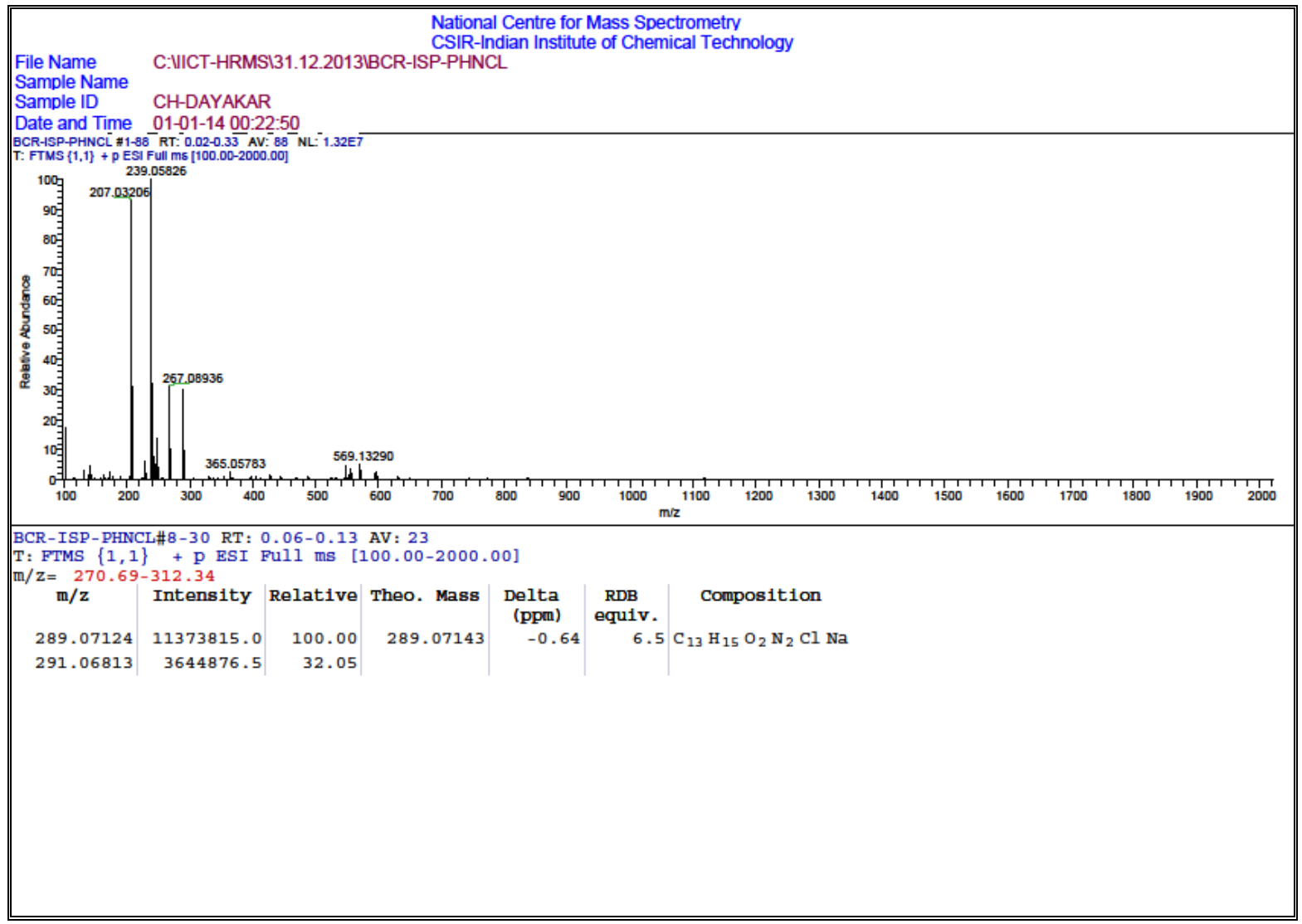

HRMS of compound 7o 


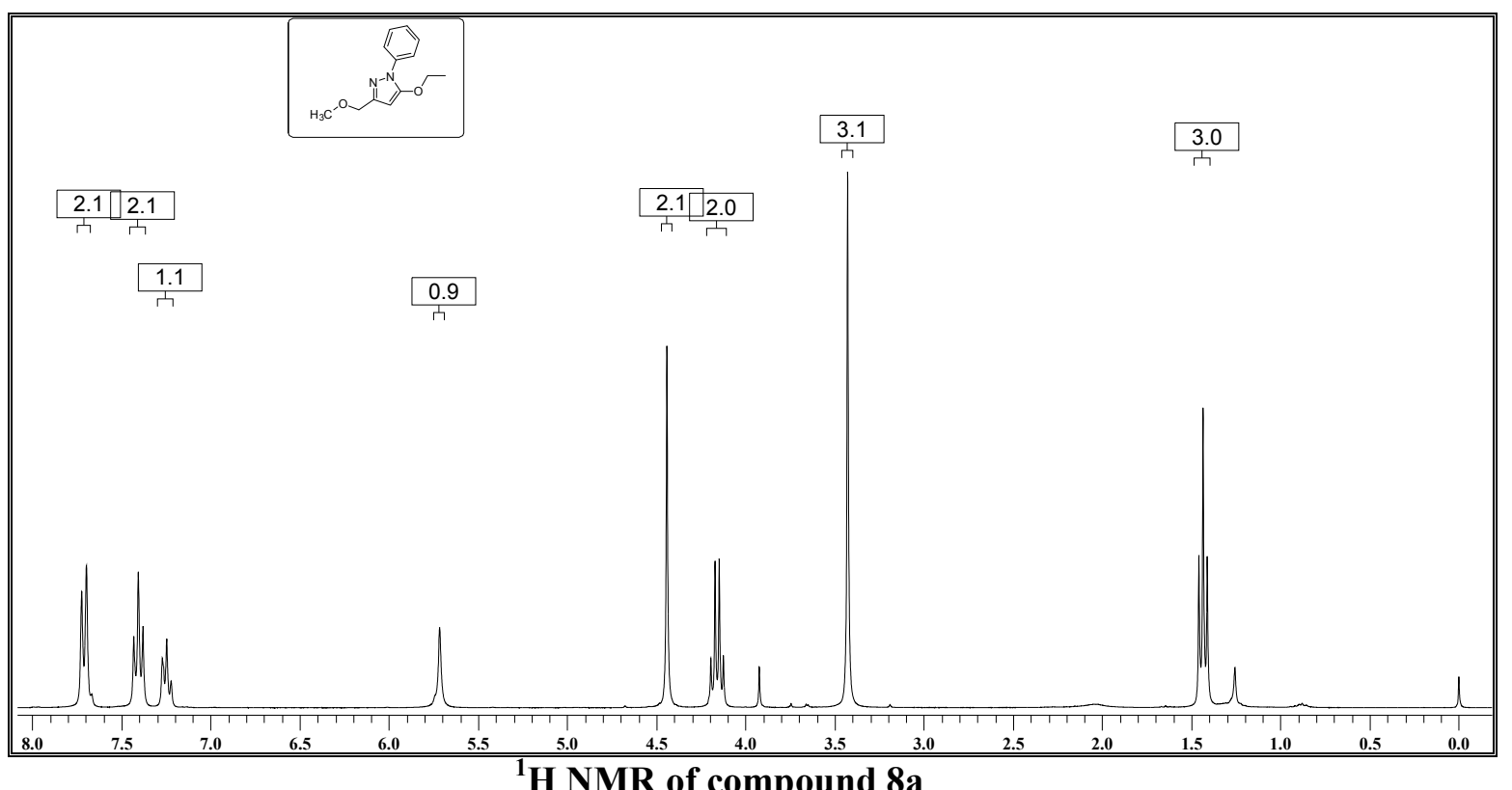

${ }^{1} \mathrm{H}$ NMR of compound 8a

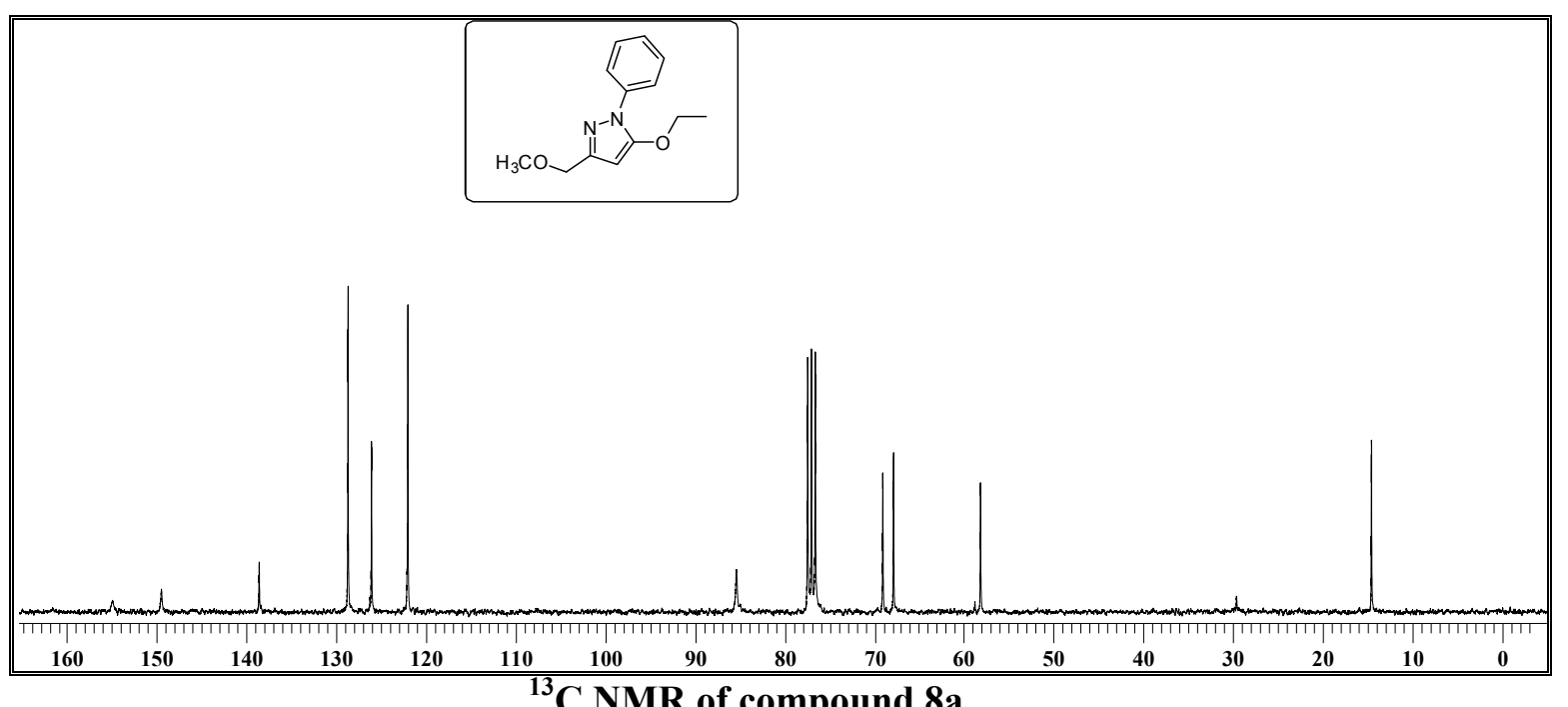




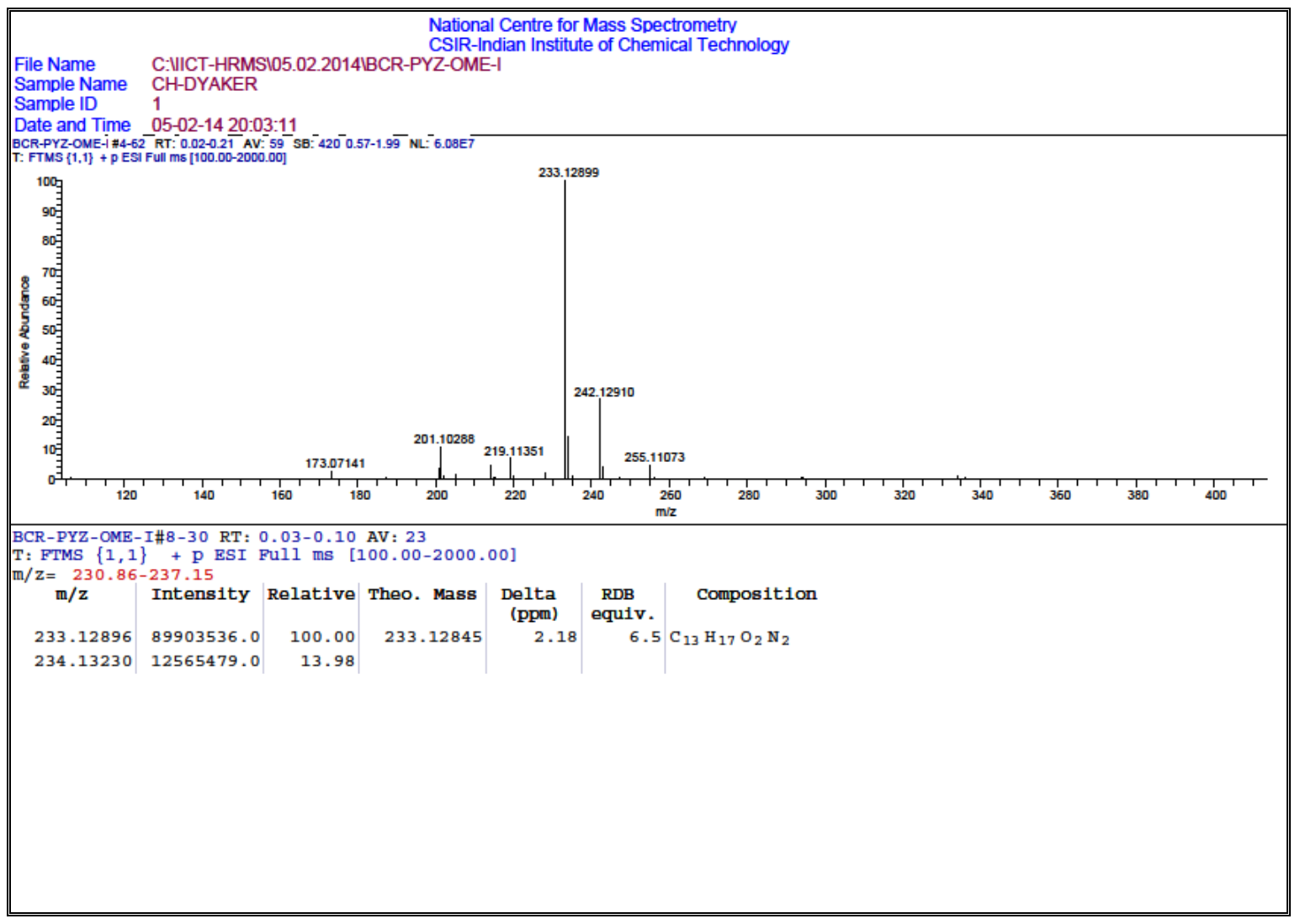

\section{HRMS of compound 8a}

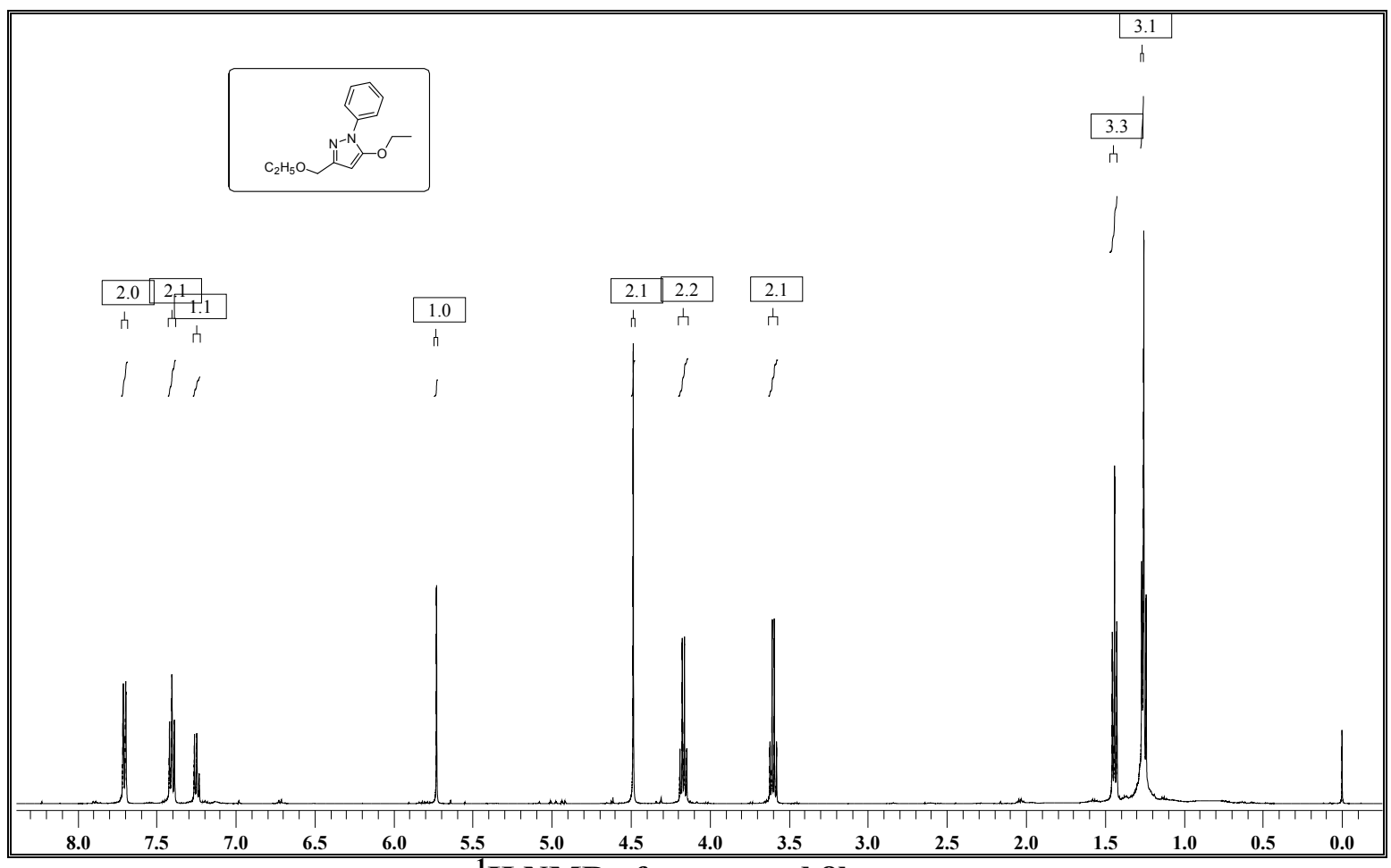

${ }^{1} \mathrm{H}$ NMR of compound $8 \mathrm{~b}$ 

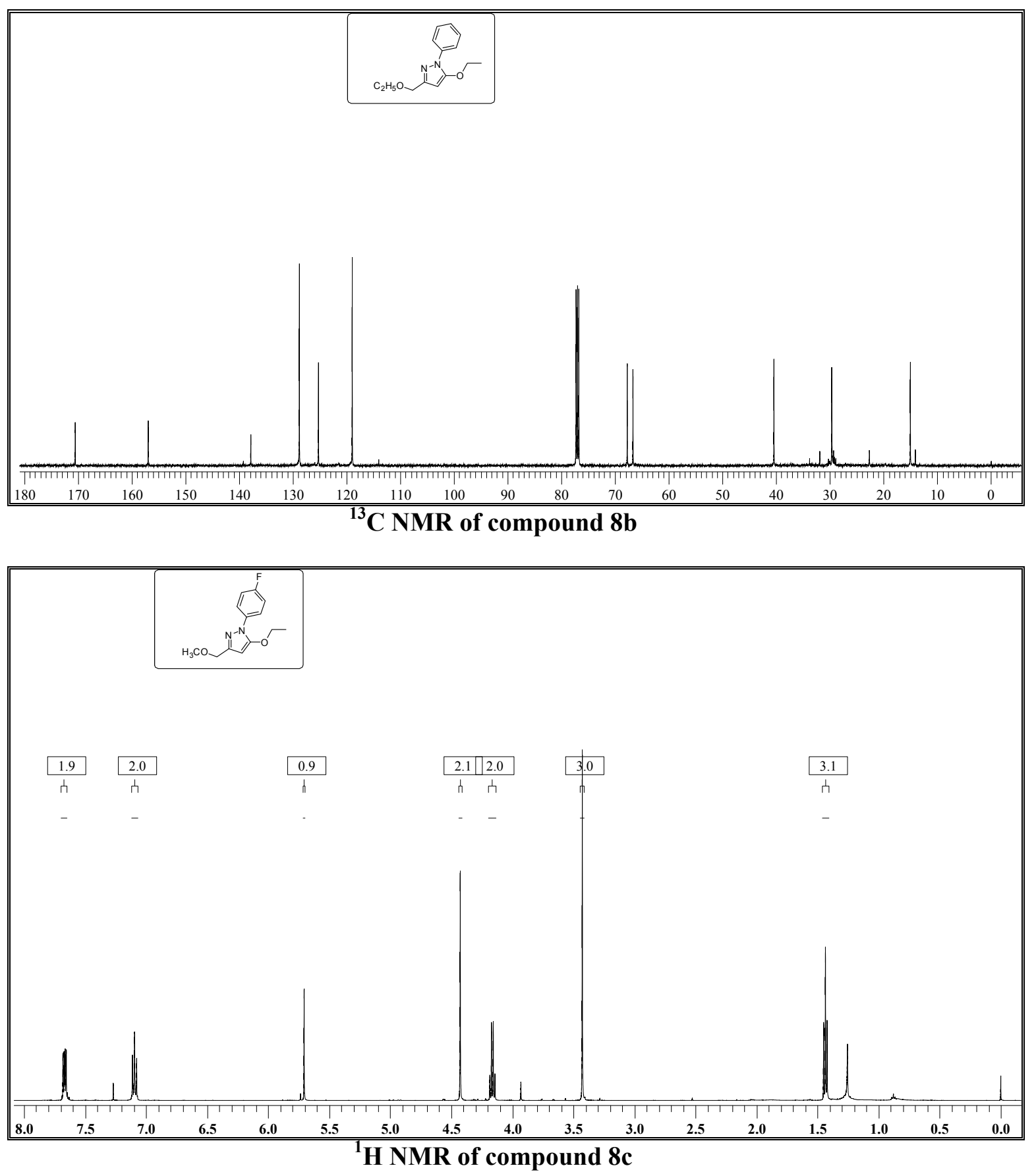


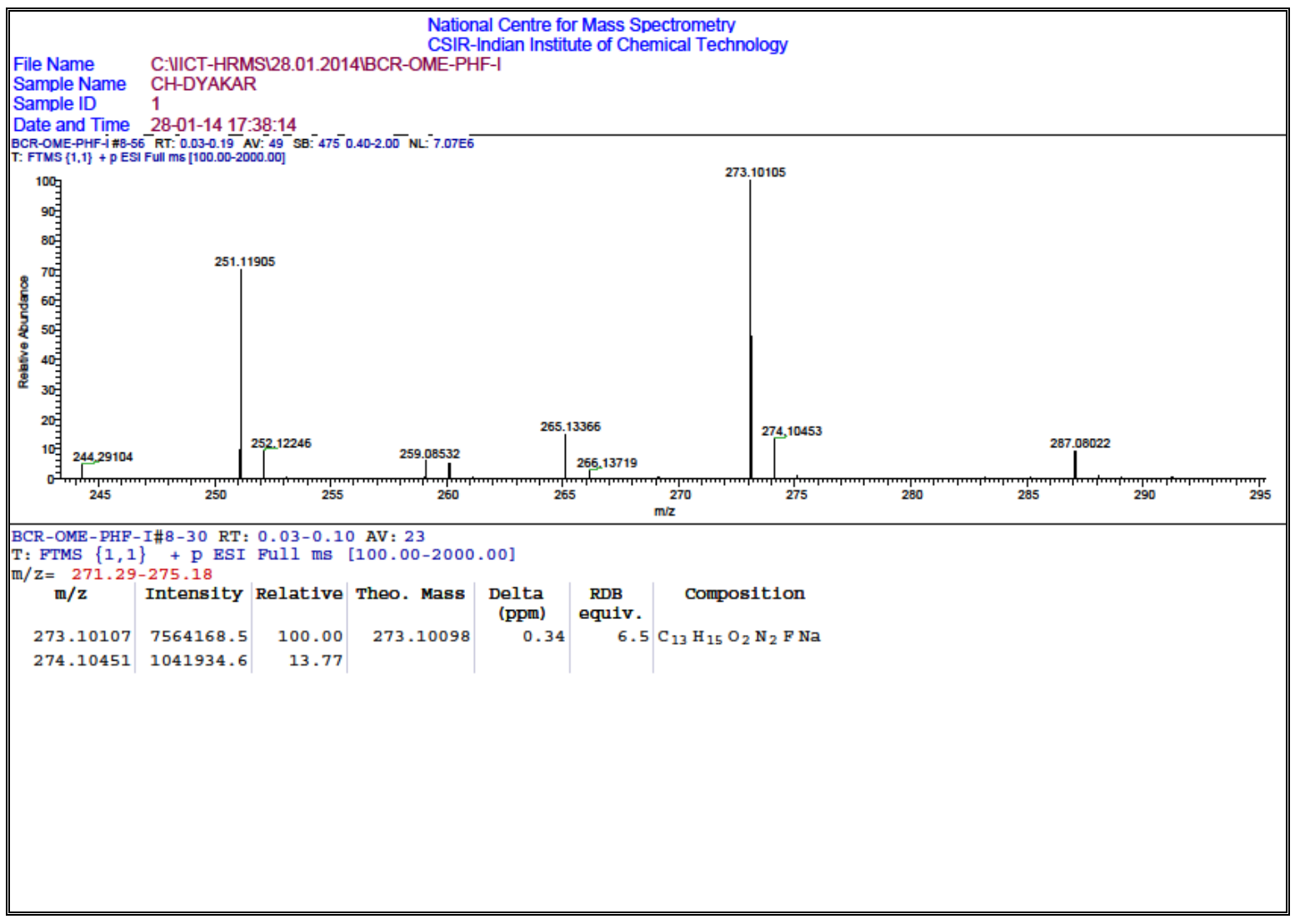

HRMS of compound 8c

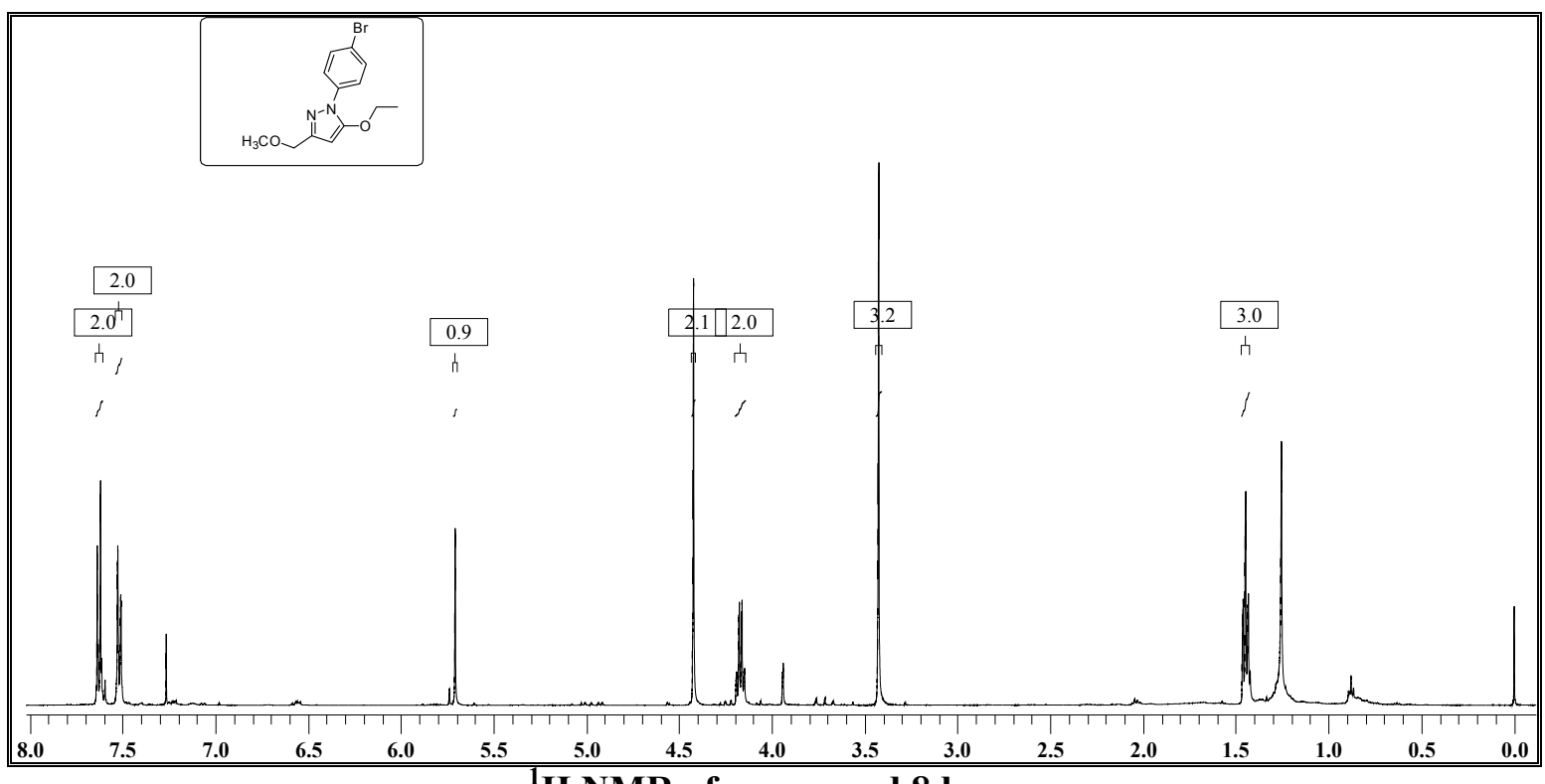

${ }^{1}$ H NMR of compound 8d 


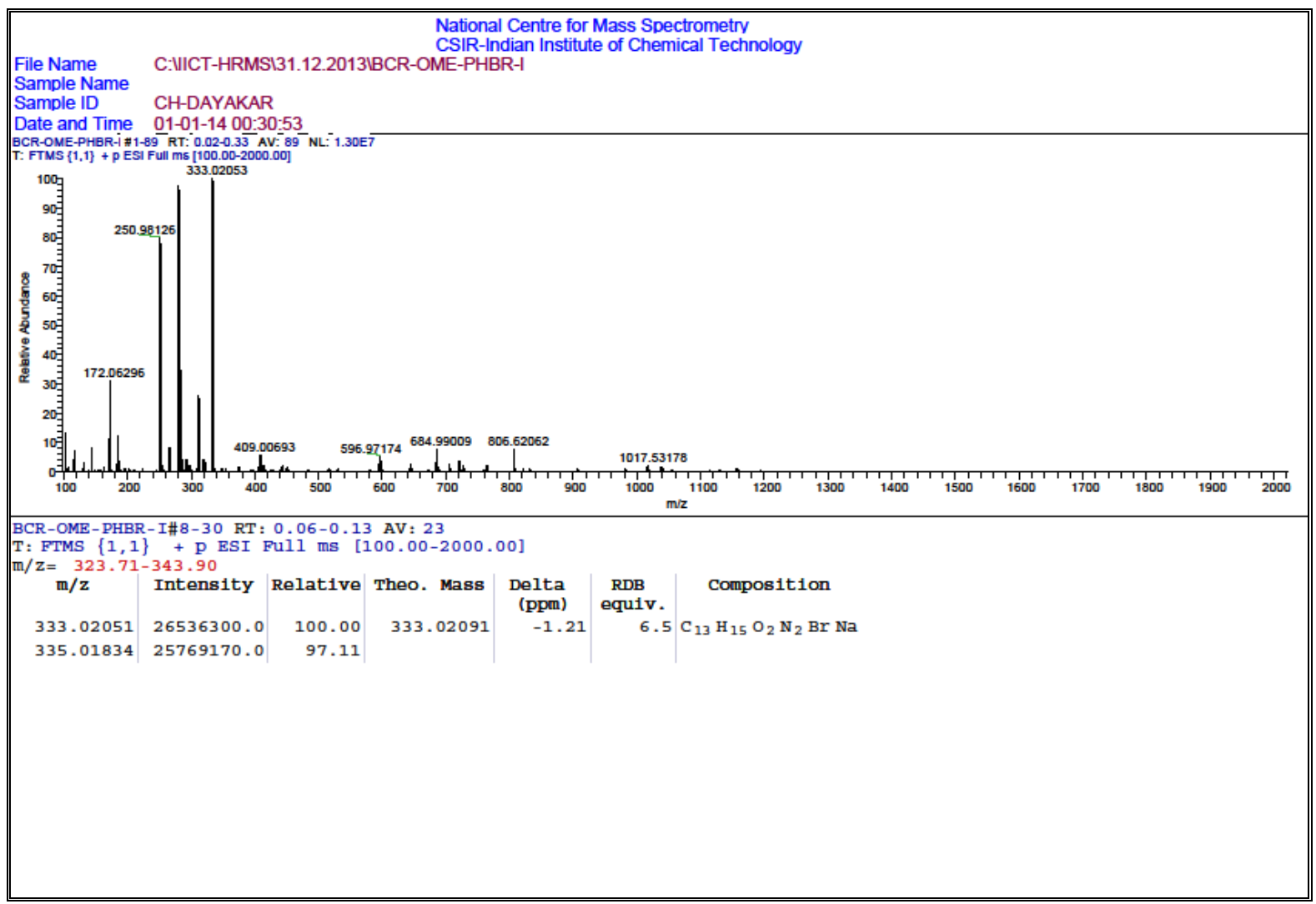

\section{HRMS of compound 8d}

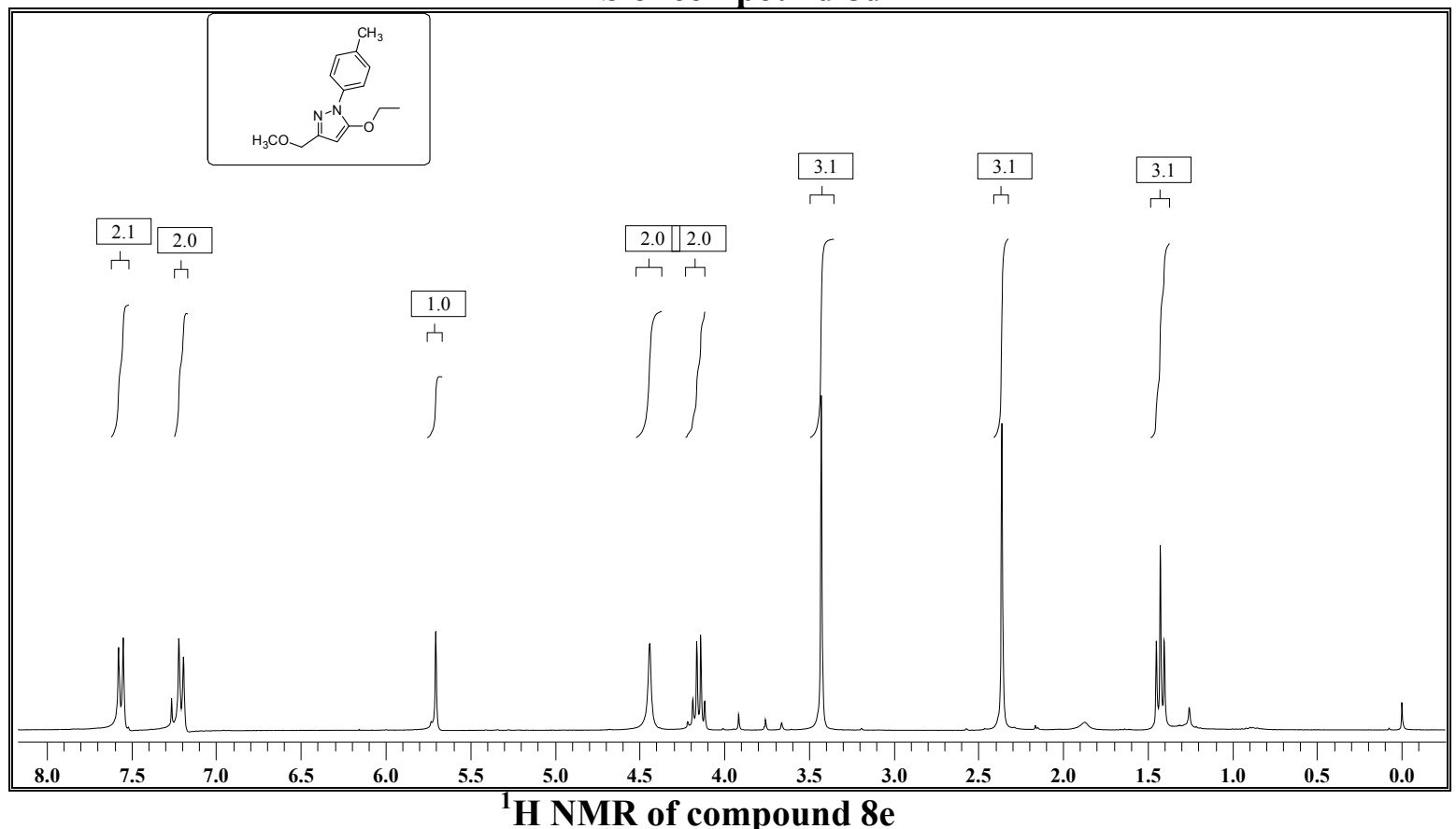



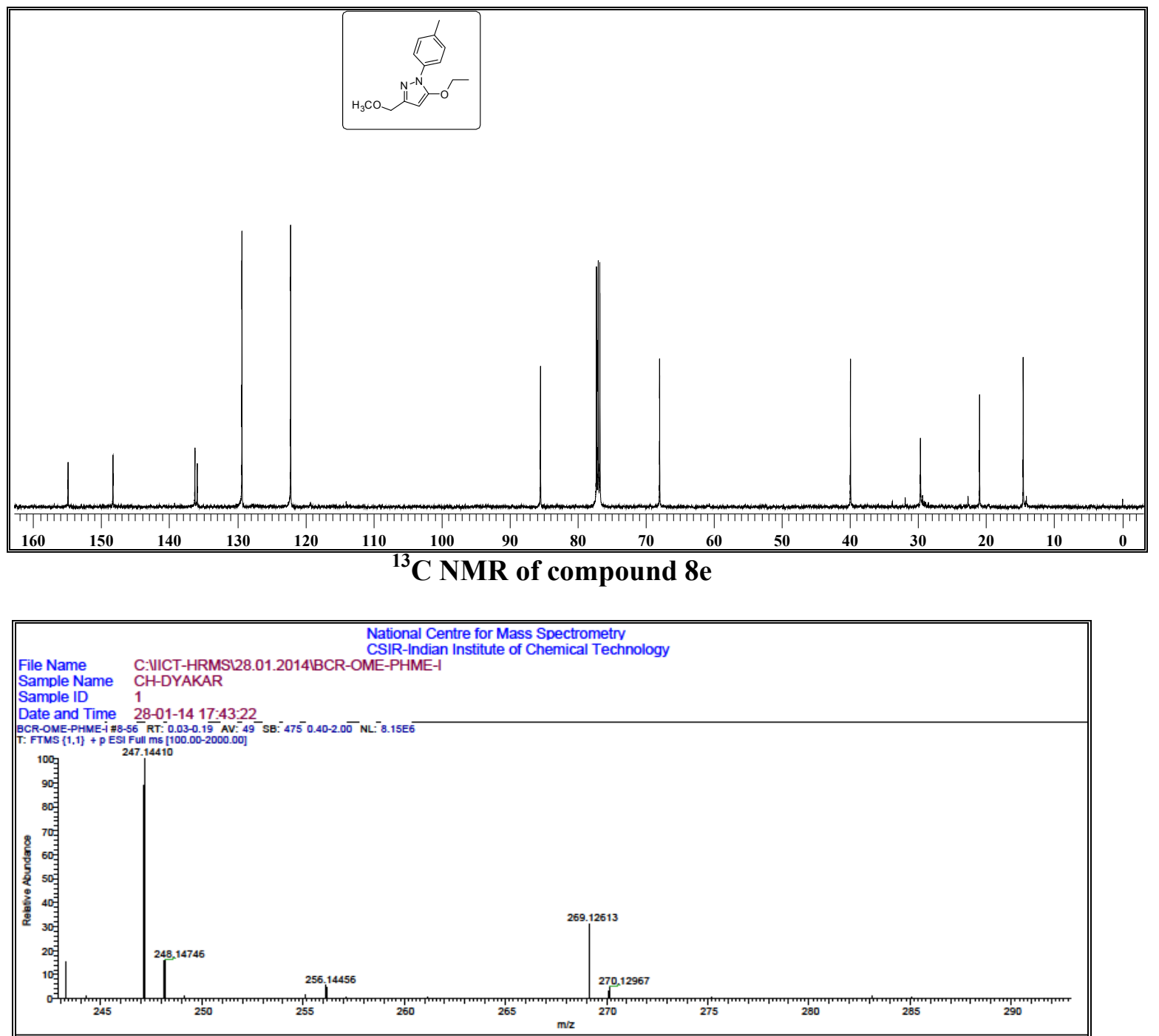

BCR-OME-PHME-I\#8-30 RT: $0.03-0.10$ AV: 23

T: FTMS $\{1,1\}+$ p ESI Fuil ms $[100.00-2000.00]$

$\mathrm{m} / \mathrm{z}=246.27-249.49$

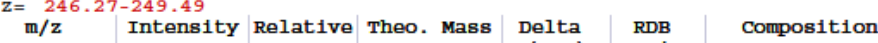

\begin{tabular}{|l|l|l|l|l|}
\hline & (ppm) equiv.
\end{tabular}

$\begin{array}{llll}247.14410 & -0.11 & 6.5 \mathrm{C}_{14} \mathrm{H}_{19} \mathrm{O}_{2} \mathrm{~N}_{2}\end{array}$

\begin{tabular}{l|r|r|}
248.14748 & 1008042.6 & 15.08
\end{tabular}

HRMS of compound 8e 

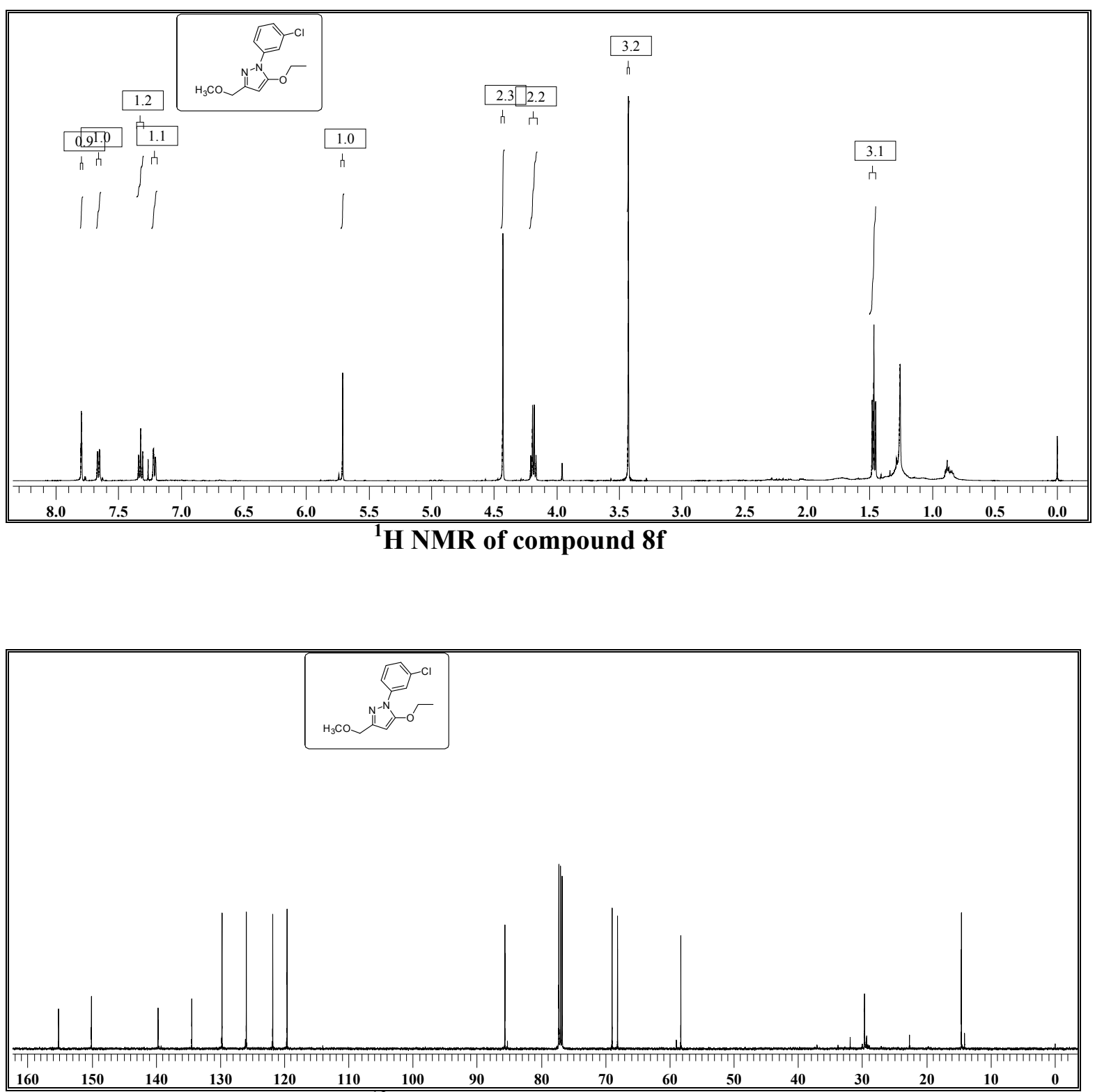

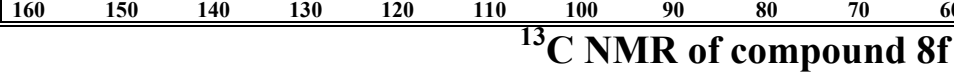




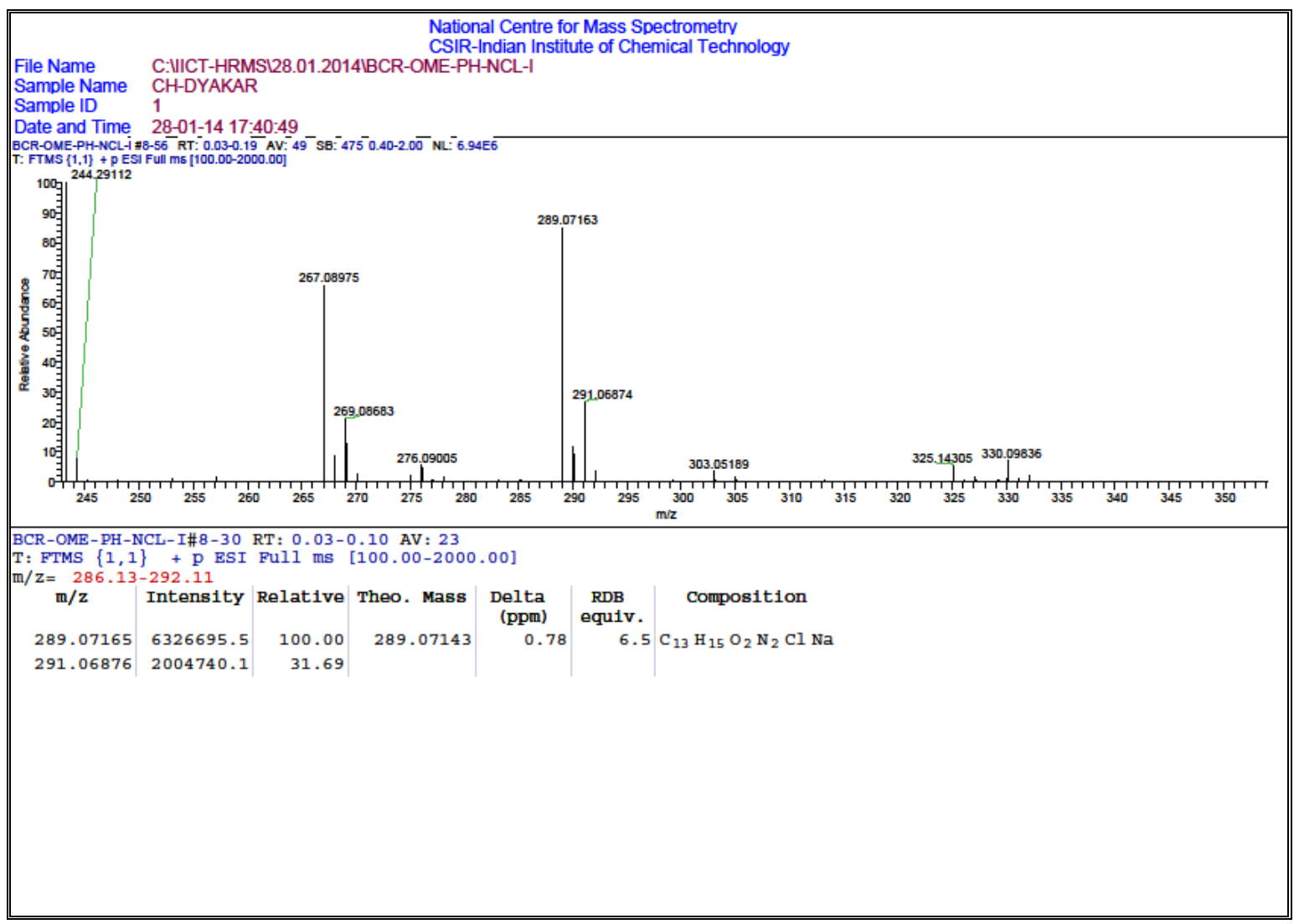

HRMS of compound 8f

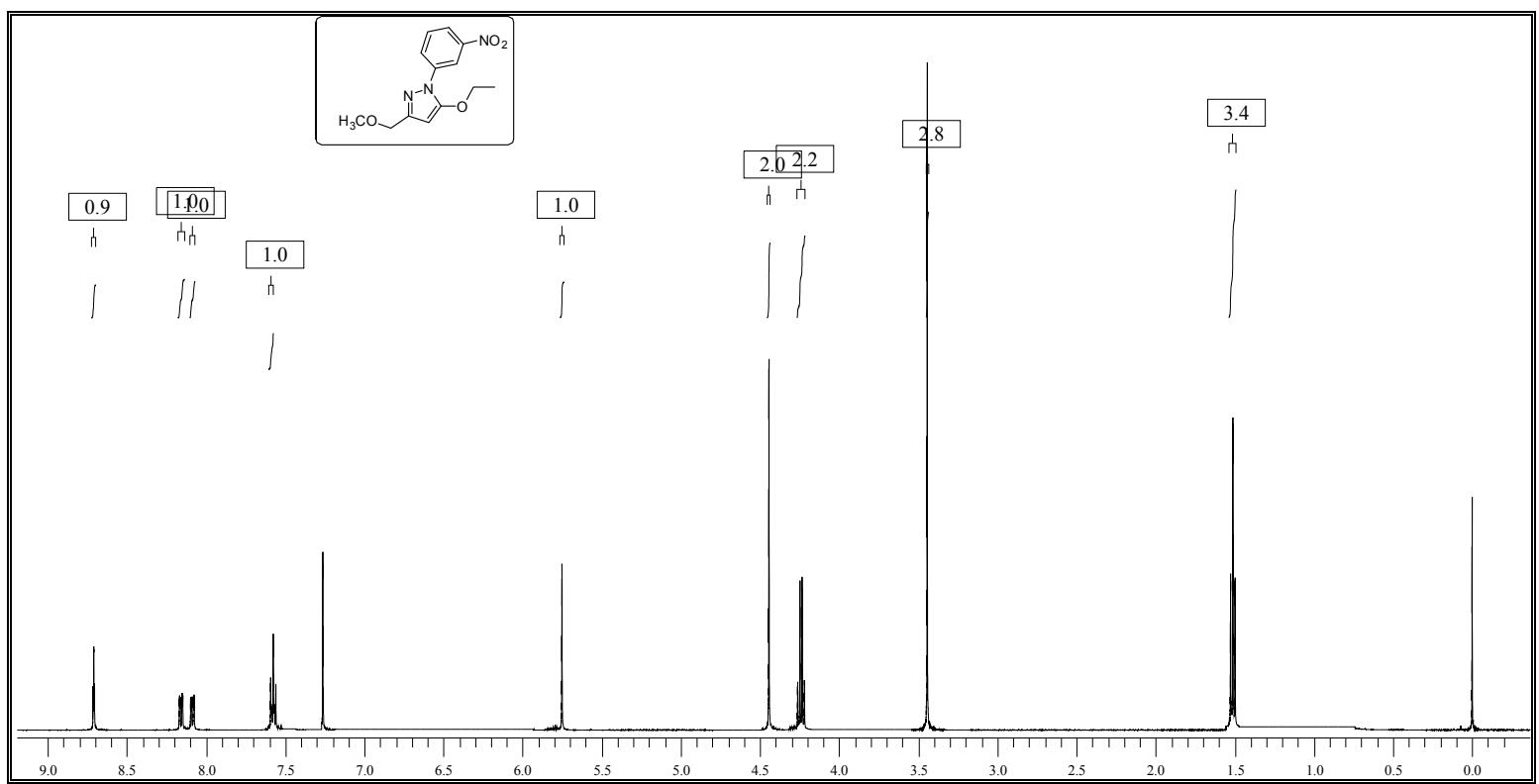

${ }^{1} \mathrm{H}$ NMR of compound $8 \mathrm{~g}$ 

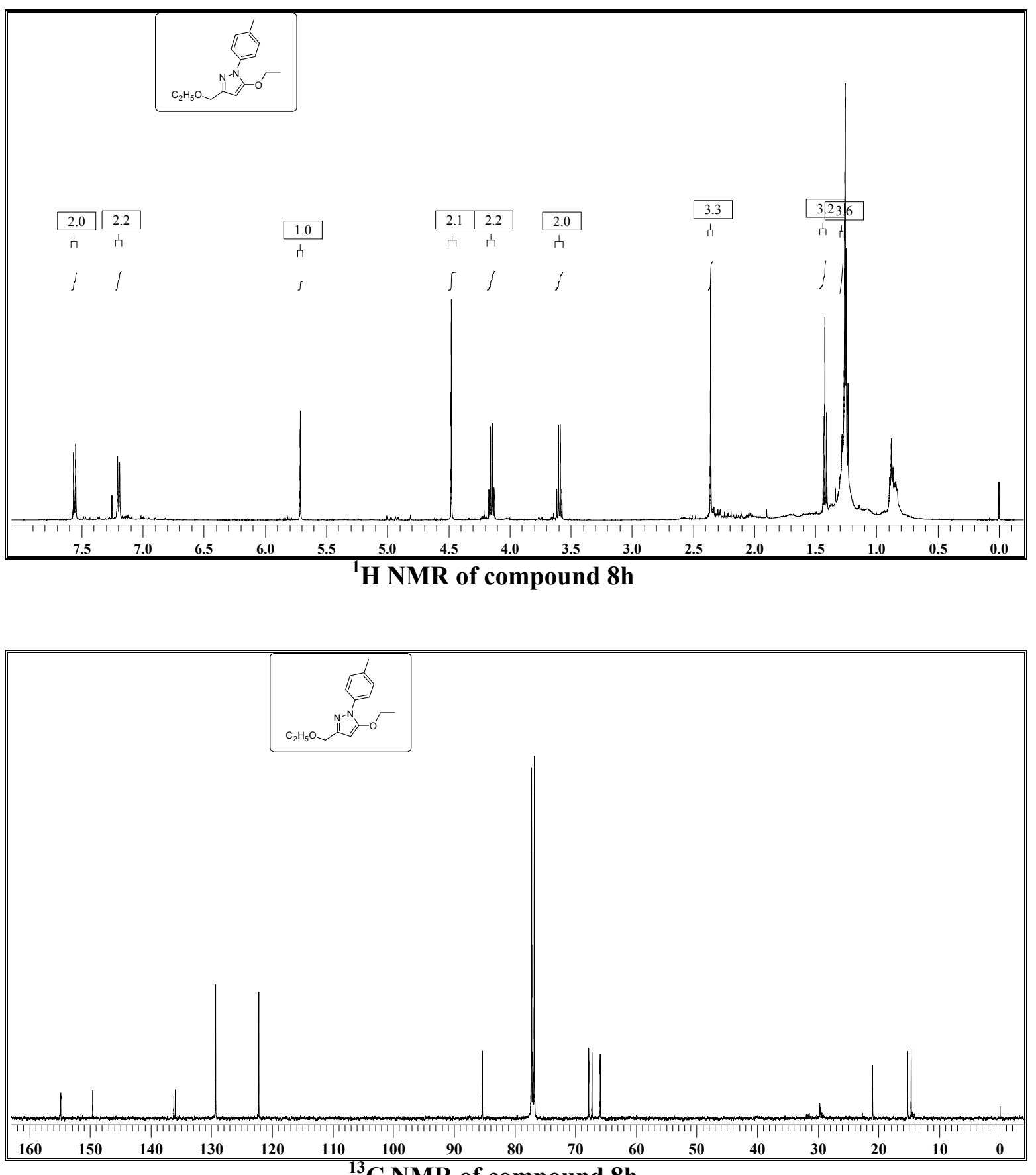

${ }^{13} \mathrm{C}$ NMR of compound $8 \mathrm{~h}$ 


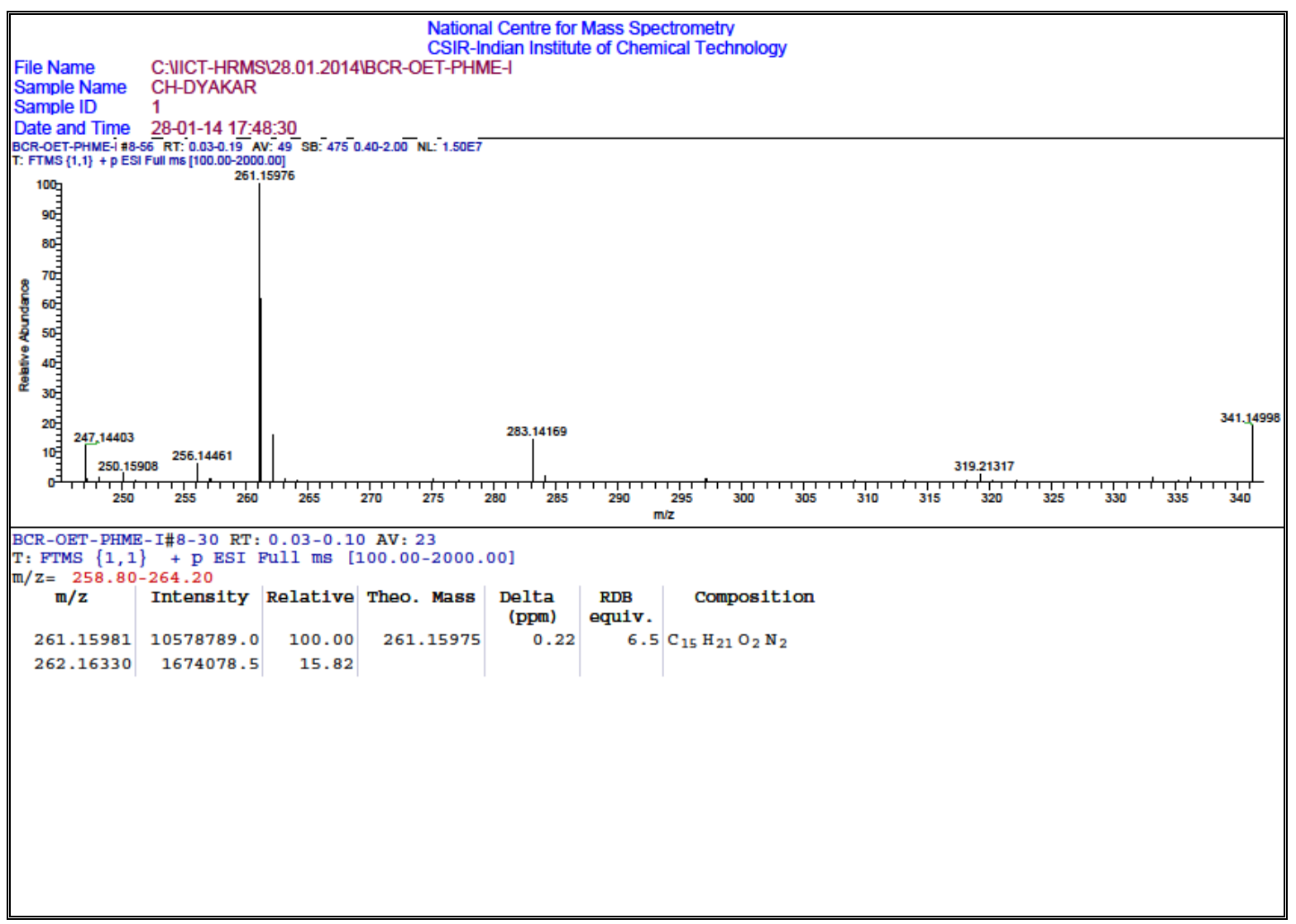

\section{HRMS of compound $8 \mathrm{~h}$}

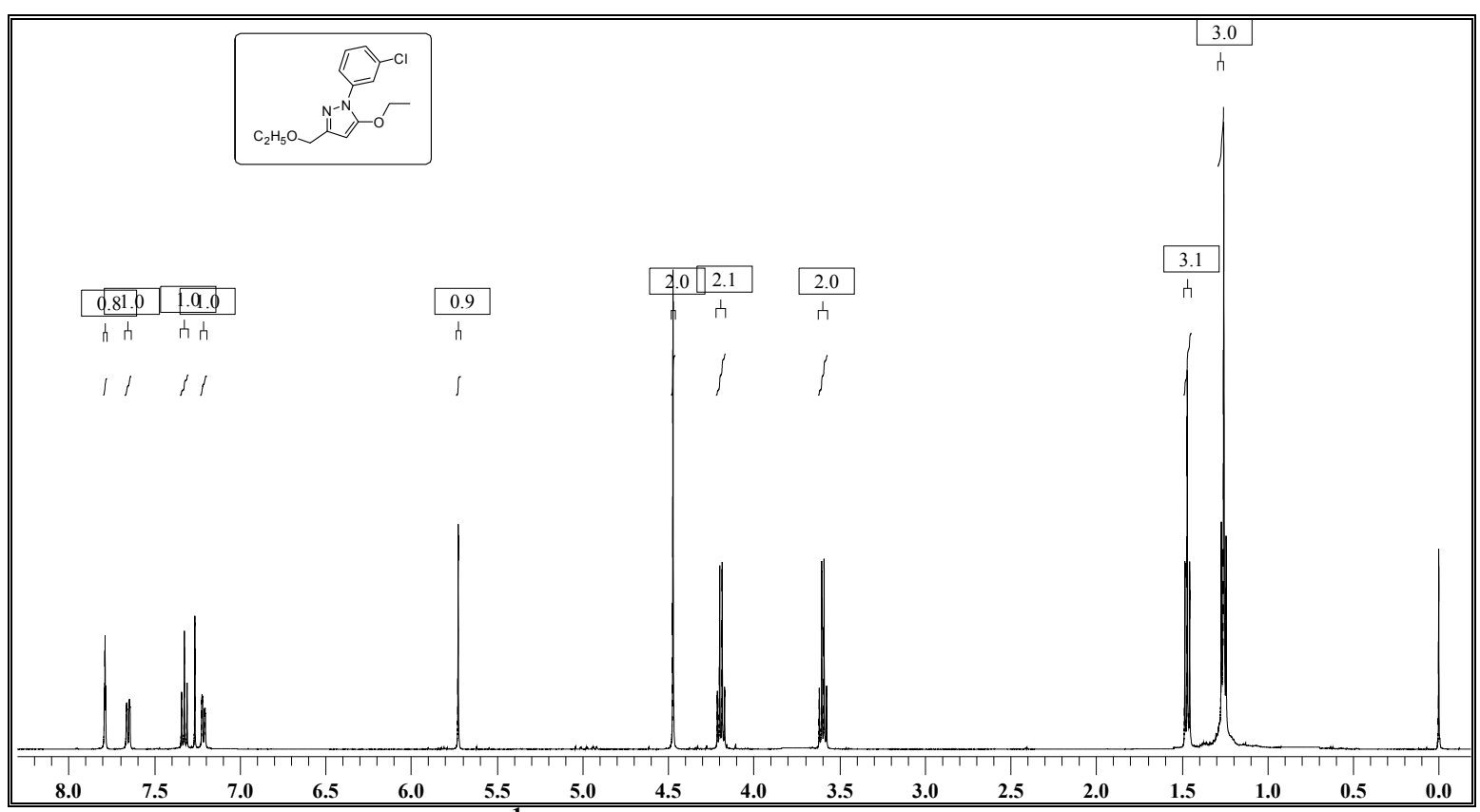

${ }^{1}$ H NMR of compound 8i 


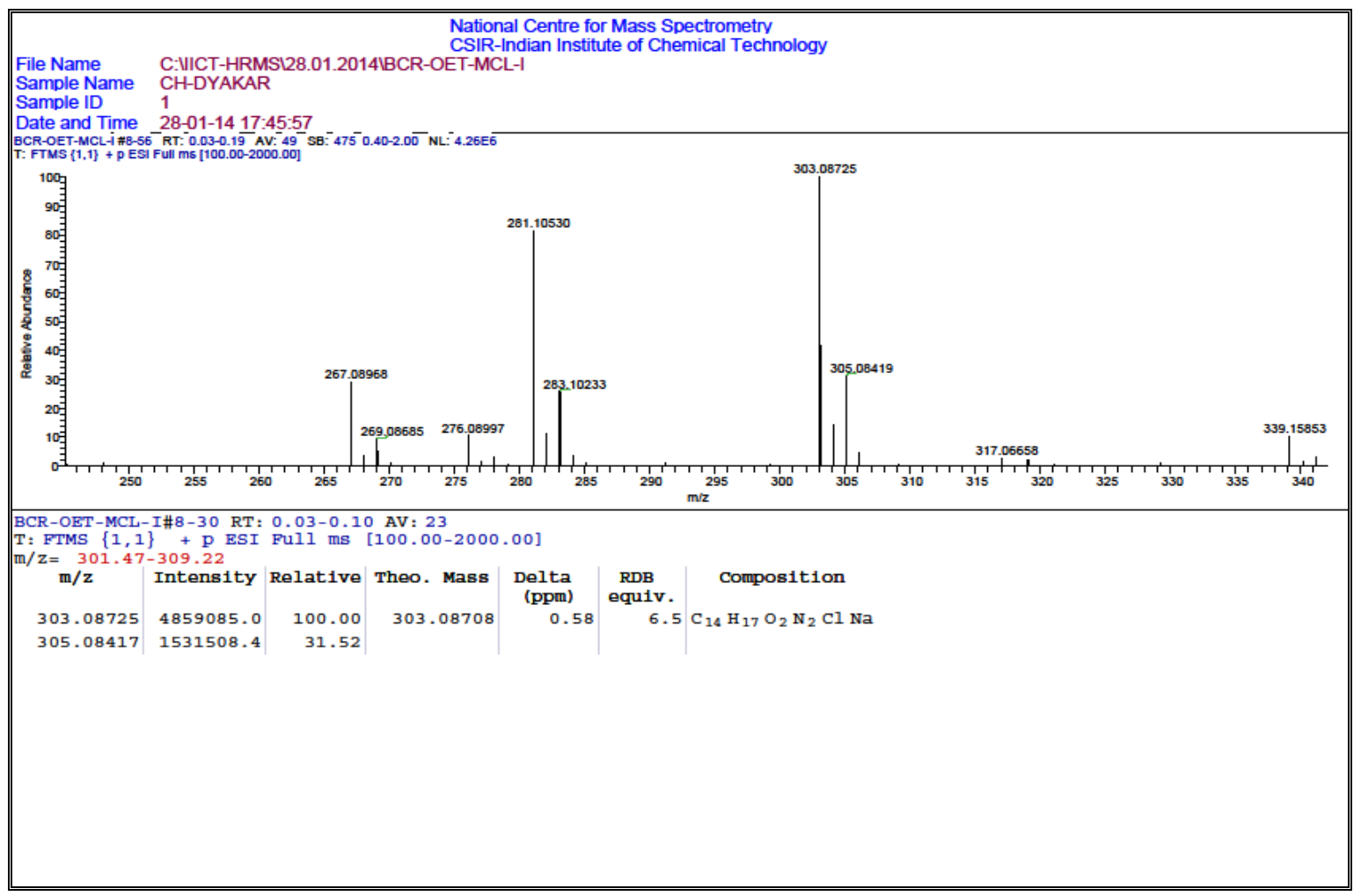

HRMS of compound 8i

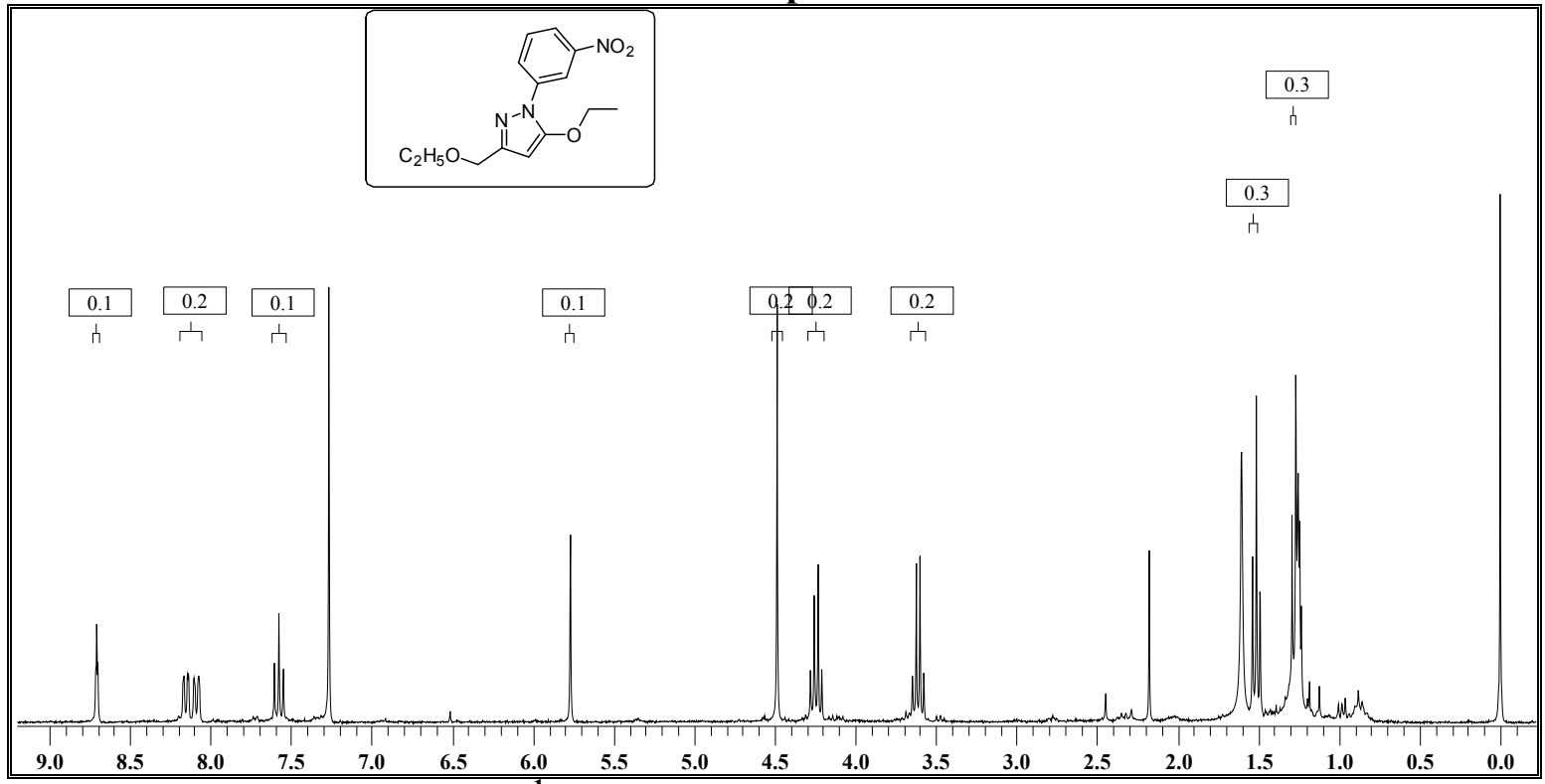

${ }^{1} \mathrm{H}$ NMR of compound $8 \mathrm{j}$ 